\title{
Bohr-Sommerfeld conditions for Integrable Systems with critical manifolds of focus-focus type
}

\author{
Vũ Ngọc San \\ September 17, 1998 \\ Mathematics Institute, \\ Budapestlaan 6, University of Utrecht, 3508 TA Utrecht, The Netherlands. \\ $\&$ \\ Institut Fourier \\ Unité mixte de recherche CNRS-UJF 5582 \\ B.P. 74, 38402 Saint-Martin d'Hères, France \\ e-mail : vu-ngoc@math.uu.nl
}

\begin{abstract}
We present a detailed study, in the semi-classical regime $h \rightarrow 0$, of microlocal properties of systems of two commuting $h$-pseudo-differential operators $P_{1}(h), P_{2}(h)$ such that the joint principal symbol $p=\left(p_{1}, p_{2}\right)$ has a special kind of singularity called a focus-focus singularity. Typical examples include the quantum spherical pendulum or the quantum Champagne bottle.

In the spirit of Colin de Verdière and Parisse [11, 12, 13], we show that such systems have a universal behavior described by singular quantization conditions of Bohr-Sommerfeld type.

These conditions are used to give a precise description of the joint spectrum of such systems, including the phenomenon of quantum monodromy and different formulations of the counting function for the joint eigenvalues close to the singularity, in which a logarithm of the semi-classical constant $h$ appears. Thanks to numerical computations done by M.S. Child for the case of the Champagne bottle, we are able to accurately illustrate our statements.
\end{abstract}

Keywords : Completely integrable Hamiltonian systems, semi-classical analysis, normal forms, Bohr-Sommerfeld conditions, microlocal solutions, non-degenerate singularities, focus-focus, monodromy, joint spectrum.

AMS Classification : 34C20, 34E20, 35P20, 57R70, 58F07, 81Q20. 


\section{Introduction}

In the long history of completely integrable systems, an important object was discovered quite recently (Duistermaat [19]) : the monodromy of the system, whose non-triviality prevents the construction of global action variables. The question about the impact of this invariant on the spectrum of quantum integrable system was raised by Cushman and Duistermaat in [15]; an answer is proposed in [40]. The issue is to describe the joint spectrum of two commuting $h-$ pseudo-differential operators $P_{1}, P_{2}$ in a region close to a critical point of the $C^{\infty}$ joint principal symbol

$$
p \stackrel{\text { def }}{=}\left(p_{1}, p_{2}\right)
$$

when the underlying Liouville integrable system $p_{1}, p_{2}$ has non trivial monodromy.

However, because of a well-known drawback of both the usual $W K B$ construction and the standard Bohr-Sommerfeld quantization conditions, the descriptions had to keep a reasonable distance away from the singular value of $p$. Here reasonable may be small but means fixed, as $h$ tends to zero. In this way, an increasing number of eigenvalues (as $h \rightarrow 0$ ) remained out of control.

On the other hand, recent achievements in semi-classical analysis of Schrödinger operators near a critical point of the potential, often via the use of microlocal normal forms, suggested that this problem should be solvable. I am referring here for instance to the work of Helffer-Robert [28], Helffer-Sjöstrand [29], Sjöstrand [38], März [33], Brummelhuis-Paul-Uribe [3], ... and in particular to the articles by Colin de Verdière and Parisse $[11,12,13]$, in which the case of a local maximum of a smooth $\left(C^{\infty}\right)$ potential for a 1-dimensional Schrödinger operator is treated. Their method rested upon a smooth normal form theorem and on the study of the hyperbolicity of the classical Hamiltonian flow.

It turns out that any two degree of freedom quantum integrable system with a focus-focus singularity exhibits at the same time a non-trivial monodromy and a hyperbolic behavior of the Hamiltonian flow. Moreover, such a singularity admits a smooth normal form, due to Eliasson [22], that has a semi-classical analogue [39]. This allows us to settle an analysis combining a geometrical description of the underlying classical completely integrable system with a microlocal analysis near the singularity.

The main result of this paper is the statement of the singular Bohr-Sommerfeld quantization conditions (theorem 6.9), which are uniform in a neighborhood of the critical value. In other words, these conditions are able to uniformly describe an increasing number of joint eigenvalues, and as such they contain the description of the quantum monodromy far from the singularity as well as the asymptotic distribution of the joint eigenvalues near the singularity.

The organization of this paper is as follows. In the first parts, the aim is to settle the ingredients needed to have a global picture of the problem. The necessary background concerning Liouville integrable systems is recalled (section 2), and the general notion of semi-classical integrable systems is explained in section 3, where we point out the role of both the principal and sub-principal symbols. The version of the microlocal tools that we will be using is given in section 4; its application to completely integrable systems is developed in the next section

(5). Besides known results concerning the WKB construction, the most fundamental results explaining our methods are probably propositions 5.1 and 5.5 . 
The rest of the paper is devoted to the case of a focus-focus singularity in a 4-dimensional cotangent bundle. Section 6 contains the microlocal analysis and the geometrical description of the monodromy that lead to our main result (theorem 6.9). Finally, we derive in section 7 the structure of the joint spectrum. Theorem 7.3 shows that one can count the number of joint eigenvalues without knowing their precise distribution near the critical value, and theorem 7.6, on the contrary, studies this distribution, by estimating the gaps between the joint eigenvalues. These statements are illustrated by the example of the Champagne bottle.

Acknowledgments. First of all, I would like to thank Y. Colin de Verdière whose numerous ideas and suggestions made this work possible. I also had stimulating discussions with J.J.Duistermaat, particularly concerning the monodromy. I would like to thank him for this and for his hospitality at Utrecht. Finally, it was a pleasure to talk with M.S.Child, whose viewpoint as a chemist was quite interesting for me (see [7]), and who accepted to provide me with a load of numerics concerning the quantum "Champagne bottle". Let him be thanked for this.

My research is supported by a Marie Curie Fellowship Nr. ERBFMBICT961572. 


\section{Table of Contents}

1 Introduction $\quad 2$

2 Classical completely integrable systems 5

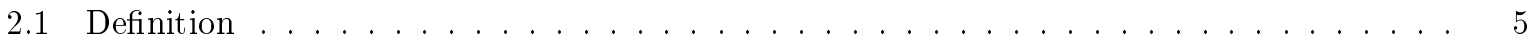

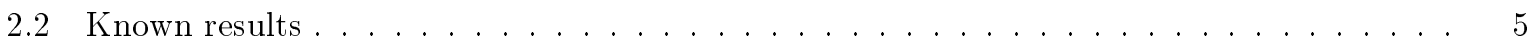

3 Semi-classical integrable systems $\quad 6$

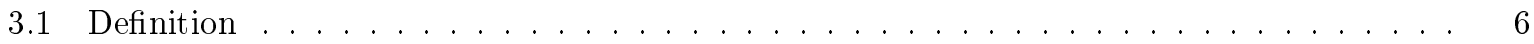

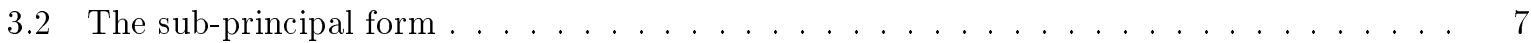

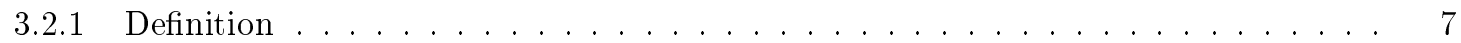

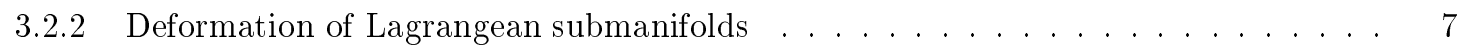

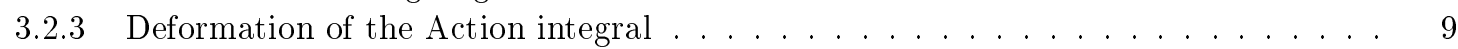

$3.2 .4 \kappa$ as a semi-classical deformation $\ldots \ldots \ldots \ldots \ldots \ldots \ldots$

4 Microlocal analysis $\quad \mathbf{1 0}$

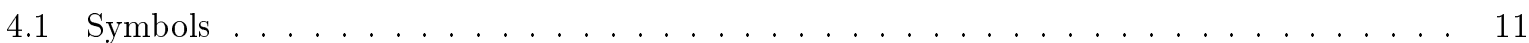

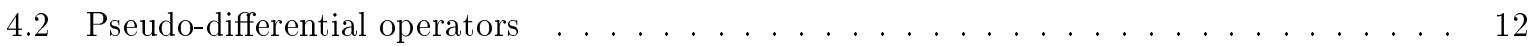

4.3 Fourier integral operators . . . . . . . . . . . . . . . . . . . . . . . 14

$4.4 h$-admissible functionals $\ldots \ldots \ldots \ldots \ldots \ldots \ldots \ldots \ldots \ldots$

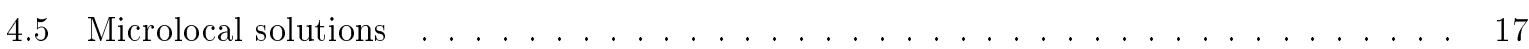

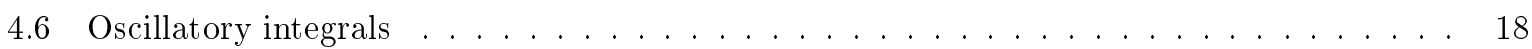

5 Non-singular quantization conditions $\quad \mathbf{2 0}$

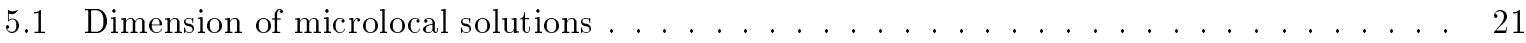

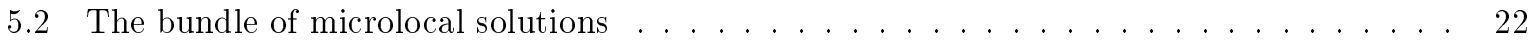

5.3 WKB method . . . . . . . . . . . . . . . . . . . . . 24

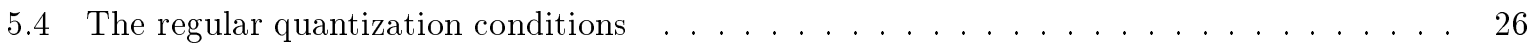

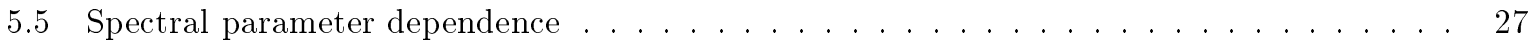

6 Bohr-Sommerfeld for a focus-focus singularity 28

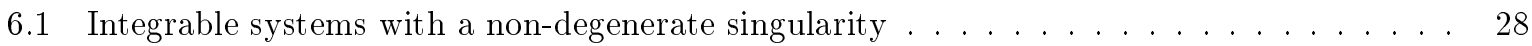

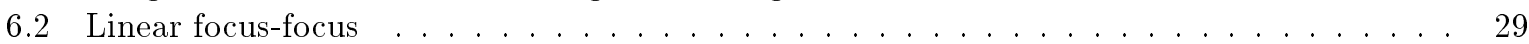

6.3 Geometry of the singular Lagrangean . . . . . . . . . . . . . . . . . . . . . . 30

6.4 Monodromy of the Lagrangean fibration around $\Lambda_{0} \ldots \ldots \ldots \ldots$

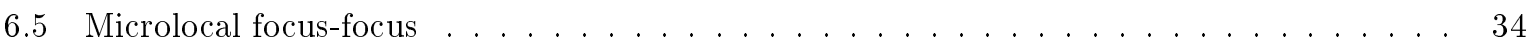

6.6 Regularization of $[\kappa] \ldots \ldots \ldots \ldots \ldots \ldots$

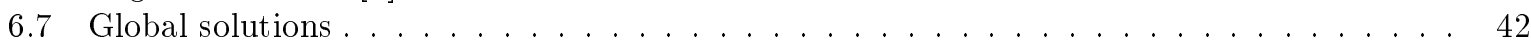

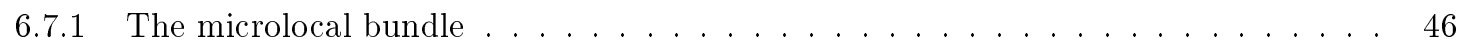

6.7 .2 The local holonomy at $m \ldots \ldots \ldots \ldots \ldots \ldots$

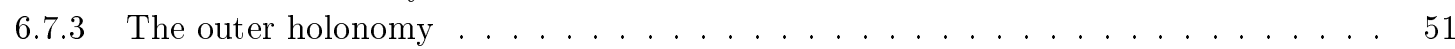

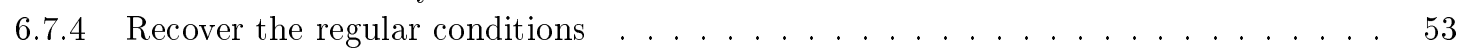

$\begin{array}{lll}7 & \text { Structure of the joint spectrum } & \mathbf{5 4}\end{array}$

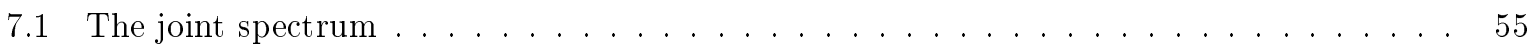

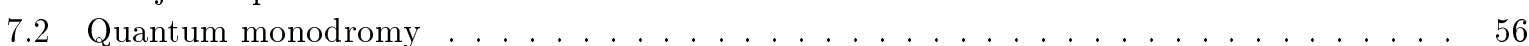

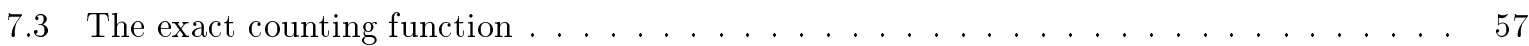

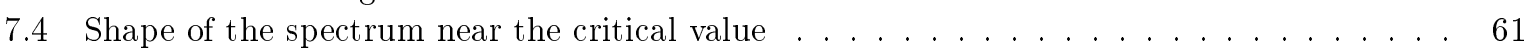

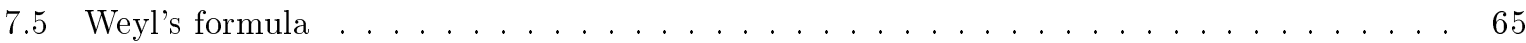




\section{Classical completely integrable systems}

\subsection{Definition}

Let $(M, \omega)$ be a symplectic manifold of dimension $2 n$. A completely (or "Liouville") integrable system on $M$ is the data of $n$ functions $f_{1}, \ldots, f_{n}$ in involution with respect to the symplectic Poisson bracket, with the requirement that their differentials $d f_{i}$ are almost everywhere independent. The function

$$
F: M \ni m \mapsto\left(f_{1}(m), \ldots, f_{n}(m)\right) \in \mathbb{R}^{n}
$$

is called the momentum map. It is indeed a momentum map for the Abelian infinitesimal action of $\mathbb{R}^{n}$ into the Lie algebra $\mathcal{X}(M)$ of Hamiltonian vector fields on $M$, given by the generators $\left(\mathcal{X}_{1}, \ldots, \mathcal{X}_{n}\right)$. Here we have denoted by $\mathcal{X}_{i}$ the Hamiltonian vector field $\mathcal{X}_{f_{i}}$ associated to $f_{i}$. The flows of these vector fields yield a local Abelian action of $\mathbb{R}^{n}$ on $M$, simply referred to in the sequel by "the action" or "the flow" of the system.

Note that each $f_{i}$, and hence every function of the form $g\left(f_{1}, \ldots, f_{n}\right)$ is locally constant under this action. The momentum map $F$ thus defines a singular fibration by invariant leaves.

These leaves $\Lambda_{c} \stackrel{\text { def }}{=} F^{-1}(c), c \in \mathbb{R}^{n}$, are generically Lagrangean submanifolds of $M$. We shall always assume that $F$ is proper, so that each $\Lambda_{c}$ is compact.

\subsection{Known results}

Local and semi-global descriptions of completely integrable systems are provided by the following facts :

- when the vector fields $\left(\mathcal{X}_{1}, \ldots, \mathcal{X}_{n}\right)$ are independent at a point $m$, the Darboux-Carathéodory theorem states that the functions $f_{i}$ can be taken as "momentum" coordinates of a symplectic chart $\{(x, \xi)\}$ on a neighborhood $U$ of $m$. In these coordinates, the foliation $\Lambda_{c} \cap U$ for $c$ close to $F(m)$ is given by $\xi=c$. The $x_{i}$ variables are therefore local coordinates for $\Lambda_{c}$.

- when $c$ is a regular value of $F$ - this includes the previous case, of course - the ArnoldLiouville theorem endows an invariant neighborhood $\Omega$ of $\Lambda_{c}$ with action-angle coordinates : $\Omega$ is symplectomorphic to a neighborhood of the zero section of the cotangent bundle $T^{*}\left(\mathbb{T}^{n}\right)$ of the $n$-torus $\mathbb{T}^{n}=\mathbb{R}^{n} / \mathbb{Z}^{n}$, and the fibration $F$ gets transformed into a smooth function of the momentum variables $\xi_{i}$ only. As a consequence, $\Lambda_{c}$, as well as the neighboring fibers, are Lagrangean tori equipped with an affine structure : the one given by the $\mathbb{R}^{n}$ action which, in these coordinates, is linear.

In the first case, the $\mathbb{R}^{n}$ action is locally free at $m$, and gives rise to a local diffeomorphism between $\left(\mathbb{R}^{n}, 0\right)$ and $\left(\Lambda_{c}, m\right)$. By transporting the standard Lebesgue measure via this diffeomorphism we obtain an invariant measure on a neighborhood of $m$ in $\Lambda_{c}$ which in DarbouxCarathéodory coordinates $(x, \xi)$ is nothing else than $|d x|$.

In the second case, this can be done globally to give a natural invariant measure $\rho_{c}$ on the whole torus $\Lambda_{c}$. Letting $m_{c}$ be the total mass of $\Lambda_{c}$, we can identify $m_{c}^{-1} \rho_{c}$ with the Lebesgue 
(or Haar) measure on the torus $\mathbb{T}^{n}$ via action-angle coordinates. $\rho_{c}$ smoothly depends on $c$ and is called the Liouville measure.

Remark 2.1. This terminology sometimes also applies to the symplectic measure $\omega^{\wedge n} / n$ ! Note that the push-forward of this measure by $F$ gives a measure $\mu_{c}$ on $\mathbb{R}^{n}$ that satisfies

$$
\frac{\omega^{\wedge n}}{n !}=\rho_{c} \otimes \mu_{c}
$$

and that is long known to be of particular importance when it comes to counting eigenvalues (see section 7). Because of the article [20] $\mu_{c}$ is sometimes called Duistermaat-Heckman's measure. $\triangle$

Since we will be dealing only with pseudo-differential quantization, we will always assume that $M$ is an open subset of a cotangent bundle $T^{*} X$, which implies that the symplectic form is exact : $\omega=d \alpha_{0}$, where $\alpha_{0}$ is the standard Liouville 1-form. If we let $i_{c}: \Lambda_{c} \hookrightarrow M$ be the inclusion, the fact that $\Lambda_{c}$ is Lagrangean implies that the 1 -form $i_{c}^{*} \alpha_{0}$ is closed and thus gives rise to an element of the cohomology $H^{1}\left(\Lambda_{c}, \mathbb{R}\right)$. With slight abuse of notation, we will still call it $\left[\alpha_{0}\right]$.

\section{Semi-classical integrable systems}

The aim of this section is to define what we consider to be the proper semi-classical quantization of completely integrable systems (in accordance with $[9,5]$ ), and to present a geometric interpretation of their sub-principal terms.

\subsection{Definition}

Let $X$ be a differentiable manifold of dimension $n$, equipped with a half-density $|d x|^{1 / 2}$, and let $\Omega$ be an open subset of $T^{*} X$. The spaces of pseudo-differential operator that are used here are defined in section 4; however, we shall here only use the fact that they are defined up to the order $O\left(h^{2}\right)$ by two functions of $\Omega$ : their principal and sub-principal symbols.

Throughout this work, pseudo-differential operators are always classical and formally selfadjoint, so that their principal and sub-principal symbols are real-valued.

A set $\left\{P_{1}(h), \ldots, P_{n}(h)\right\}$ of $n$ pseudo-differential operators in $\Psi^{0}(\Omega)$ of order zero is called a semi-classical integrable system if :

- the principal symbols $p_{1}, \ldots, p_{n}$ form a completely integrable system.

- $\forall i, j \quad\left[P_{i}(h), P_{j}(h)\right]=0$.

Note that the second condition already implies that the principal symbols are in involutions.

Because of this definition any semi-classical integrable system has an underlying classical completely integrable system, which means that the main geometric ingredient of such a system is the Lagrangean fibration $\Lambda_{c}$ given by the principal symbols. Of particular importance for us will be the principal Lagrangean $\Lambda_{0}$. 
Remark 3.1. All the results of sections 2-6 would still hold if the commutation property is weakened to $\left[P_{i}(h), P_{j}(h)\right]=O\left(h^{\infty}\right)$. Section 7 however requires the exact commutation of the operators, in order to define their joint spectrum. Similarly, a number of results before that section would still be valid if the self-adjointness requirement is dropped and replaced by the assumption that both the principal and sub-principal symbols are real-valued.

\subsection{The sub-principal form}

\subsubsection{Definition}

Apart from the principal symbols, the data of a semi-classical integrable system defines another set of functions, namely the sub-principal symbols $r_{1}, \ldots, r_{n}$. We assume here that these functions are real-valued. While the principal symbols gave rise to the momentum map and its Lagrangean fibration, the sub-principal symbols will be viewed as characteristic of an infinitesimal deformation of the Lagrangean leaves.

Definition 3.1 The sub-principal form $\kappa_{c}$ of a semi-classical integrable system is the differentiable 1-form on $\Lambda_{c}$ defined at non-singular points of $F$ by:

$$
\kappa_{c}\left(\mathcal{X}_{i}\right)=-r_{i}
$$

Recall that $\mathcal{X}_{i}$ denote the Hamiltonian vector field of $p_{i}$. At such a non-singular point, $\left(\mathcal{X}_{1}, \ldots, \mathcal{X}_{n}\right)$ is a basis of the tangent space of $\Lambda_{c}$.

This form also appears in [6].

The first property of $\kappa_{c}$ is the fact that it is closed. Indeed, the $r_{i}$ 's are not just any functions. The Weyl rule (section 4, formula (4)) applied to the commutation property of the pseudo-differential operators $P_{i}$ is, at the order $h^{2}$, equivalent to :

$$
\left\{r_{i}, p_{j}\right\}=\left\{r_{j}, p_{i}\right\}
$$

In a symplectic chart given by the Darboux-Carathéodory theorem, this reads :

$$
\frac{\partial r_{i}}{\partial x_{j}}=\frac{\partial r_{j}}{\partial x_{i}}
$$

and $\kappa_{c}$ is the closed 1 -form $\kappa_{c}=-\sum r_{i} d x_{i}$.

\subsubsection{Deformation of Lagrangean submanifolds}

Let $\mathcal{L}(M)$ be the set of all Lagrangean submanifolds of a $2 n$ dimensional symplectic manifold $M$. By the Darboux-Weinstein theorem, we can identify a tubular neighborhood of a Lagrangean submanifold $\Lambda_{0}$ with a neighborhood of the zero section of $T^{*} \Lambda_{0}$. Any other Lagrangean submanifold within that neighborhood can then be identified with a closed 1-form on $\Lambda_{0}$ (see [41]). This gives a "chart" for $\mathcal{L}(M)$, making it formally a differentiable infinite dimensional manifold, whose tangent space at $\Lambda_{0}$ is naturally identified with the space of closed 1-forms on $\Lambda_{0}$. 
One can be more specific. The exponential map for vector fields gives a diffeomorphism between a tubular neighborhood of $\Lambda_{0}$ and the normal bundle $\frac{T_{\Lambda_{0}} M}{T \Lambda_{0}}$. The latter is identified with $T^{*} \Lambda_{0}$ by means of the symplectic form : let $x \in \Lambda_{0}$; to any $X \in T_{x} M$ is associated the cotangent vector

$$
\tilde{\omega}(X)=i_{X} \omega_{\mid T_{x} \Lambda_{0}}
$$

Since $\Lambda_{0}$ is Lagrangean, the kernel of $\tilde{\omega}$ is exactly $T_{x} \Lambda_{0}$.

In this way, we can easily describe the tangent space of $\mathcal{L}(M)$ : an infinitesimal variation of $\Lambda_{0}$ is by definition a vector field transversal to $\Lambda_{0}$, that is, a section of $\frac{T_{\Lambda_{0}} M}{T \Lambda_{0}}$. By the above isomorphism, it can be identified with a 1 -form on $\Lambda_{0}$. That infinitesimal variation is performed within the space of Lagrangean submanifolds if and only if this 1-form is closed, that is, the deformation vector field is locally Hamiltonian (see also [42]).

Let us apply this description to the case where a path in $\mathcal{L}(M)$ is given as a 1-parameter family of level sets of $n$ independent functions $p_{i}^{t}$ in involution :

$$
\Lambda^{t}=\left\{p_{i}^{t}=0 .\right\}
$$

Lemma 3.1 The infinitesimal variation 1-form $\kappa=\frac{d}{d t} \Lambda_{\mid t=0}^{t}$ is given by

$$
\kappa\left(\mathcal{X}_{p_{i}^{0}}\right)=-\frac{\partial p_{i}^{t}}{\partial t}{ }_{\mid t=0}
$$

Proof. Let $x \in \Lambda^{0}$. Complete $\left(p_{1}^{0}, \ldots, p_{n}^{0}\right)$ into a Darboux-Carathéodory chart, so that the tangent vectors $\frac{\partial}{\partial p_{j}^{0}}$ form a basis of the normal bundle. Taylor's formula $p_{i}^{t} \sim p_{i}^{0}+\left.t \frac{\partial p_{i}^{t}}{\partial t}\right|_{t=0}$ shows that at $t=0$, the deformation vector field for $\Lambda^{t}$ is given by

$$
X(x)=\sum_{j}-\frac{\partial p_{i}^{t}(x)}{\partial t}{ }_{\mid t=0} \frac{\partial}{\partial p_{j}^{0}} .
$$

To this transversal vector field corresponds the 1 -form $\kappa=i_{X} \omega$. On the basis $\left(\mathcal{X}_{p_{1}^{0}}, \ldots, \mathcal{X}_{p_{n}^{0}}\right)$ of $T \Lambda^{0}$, it is given by $\kappa\left(\mathcal{X}_{p_{i}^{0}}\right)=\omega\left(X, \mathcal{X}_{p_{i}^{0}}\right)=d p_{i}^{0} \cdot X=-\left.\frac{\partial p_{i}^{t}}{\partial t}\right|_{t=0}$.

Remark 3.2. For each $t$, the functions $p_{1}^{t}, \ldots, p_{n}^{t}$ define a completely integrable system in a neighborhood of $\Lambda^{t}$. The variation 1 -form can be computed not only for $\Lambda^{t}$, but also for the neighboring leaves $\Lambda_{c}^{t} \stackrel{\text { def }}{=} \cap_{i}\left(p_{i}^{t}\right)^{-1}(c)$. Then $\kappa_{c}=\frac{d}{d t} \Lambda_{c \mid t=0}^{t}$ is given on $\Lambda_{c}$ as in lemma 3.1 :

$$
\kappa_{c}\left(\mathcal{X}_{p_{i}^{0}}\right)=-\frac{\partial p_{i}^{t}}{\partial t}{ }_{\mid t=0}
$$

The fact that for all $c, \kappa_{c}$ is closed can be checked by differentiating the equality $\left\{p_{i}^{t}, p_{j}^{t}\right\}=0$ at $t=0$.

Remark 3.3. It is natural now to be willing to consider the level sets

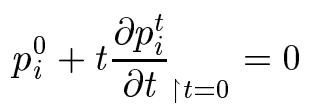


as a "linearization" of the family $\Lambda_{t}$. Unfortunately, these level sets are in general not Lagrangean. More precisely, the symplectic form restricted to them is of order $t^{2}$. However, one can prove that there exists a set of functions $\tilde{r}_{1}, \ldots, \tilde{r}_{n}$ such that the functions

$$
\tilde{p}_{i}^{t} \stackrel{\text { def }}{=} p_{i}^{0}+t \tilde{r}_{i}
$$

are indeed in involution, and such that the corresponding deformation 1-forms $\tilde{\kappa}_{c}$ satisfies

$$
\left[\widetilde{\kappa}_{c}\right]=\left[\kappa_{c}\right] \in H^{1}\left(\Lambda_{c}^{0}\right) .
$$

The value of $\tilde{r}_{1}$ on $\Lambda_{c}$ is constant and obtained by averaging the sub-principal terms $\frac{\partial p_{i}^{t}}{\partial t}\lceil t=0$ over $\Lambda_{c}$.

\subsubsection{Deformation of the Action integral}

Let $\Lambda_{t}$ be a smooth family of Lagrangean submanifolds of $M$, and let $\gamma_{t}(\theta), \theta \in S^{1}$ be a smooth family of loops such that each $\gamma_{t}$ is drawn on $\Lambda_{t}$. Suppose that on a neighborhood of the image of $\gamma_{0}$, the symplectic form $\omega$ is exact : $\omega=d \alpha$. Then this holds for $t$ small enough, and we can define the action integral :

$$
A\left(\gamma_{t}\right)=\int_{\gamma_{t}} \alpha
$$

Lemma 3.2 The variation 1-form $\kappa=\frac{d}{d t} \Lambda_{t \mid t=0}$ on $\Lambda_{0}$ is characteristic of the infinitesimal variation of the action, in the following sense:

$$
\frac{d}{d t} A\left(\gamma_{t}\right)_{\mid t=0}=\int_{\gamma_{0}} \kappa
$$

Proof. We want to prove that the 1 -forms $\frac{d}{d t}\left(\gamma_{t}^{*}(\alpha)\right)_{\lceil t=0}$ and $\gamma_{0}^{*}(\kappa)$ are cohomologous on $S^{1}$. We have

$$
\begin{aligned}
& \frac{d}{d t}\left(\gamma_{t}^{*}(\alpha)\right)=\gamma_{t}^{*}\left(\mathcal{L}_{\frac{\partial \gamma_{t}}{\partial t}} \alpha\right)= \\
& =\gamma_{t}^{*}\left(i_{\frac{\partial \gamma_{t}}{\partial t}} d \alpha+d\left(i_{\frac{\partial \gamma_{t}}{\partial t}} \alpha\right)\right) .
\end{aligned}
$$

At $t=0$, the vector field $\frac{\partial \gamma_{t}}{\partial t}$ splits into two components $X_{\tau}$ and $X_{\nu}$, the first one being tangent to $\Lambda_{0}$. The other one, in the normal bundle $\frac{T_{\Lambda_{0}} M}{T \Lambda_{0}}$, is by definition the deformation vector field of the family $\Lambda_{t}$. Therefore,

$$
\gamma_{0}^{*}\left(i_{\frac{\partial \gamma_{t}}{\partial t}} d \alpha\right)=\gamma_{0}^{*}\left(i_{X_{\tau}} \omega\right)+\gamma_{0}^{*}\left(i_{X_{\nu}} \omega\right)=0+\gamma_{0}^{*}(\kappa)
$$

which gives the result.

Remark 3.4. Of course, the value $t=0$ plays a arbitrary role : if we define $\kappa_{t}=\frac{d}{d t} \Lambda_{t}$, then $\frac{d}{d t} A\left(\gamma_{t}\right)=\int_{\gamma_{t}} \kappa_{t}$ 


\subsection{4 $\kappa$ as a semi-classical deformation}

Returning to the hypothesis of paragraph (3.2.1), we are now able to give a geometrical interpretation of the sub-principal form.

The total symbol $p_{i}(h)$ of $P_{i}(h)$ is considered as a semi-classical deformation of the principal symbol $p_{i}^{0}$. Then around any regular point of the principal symbol, the sub-principal form is equal to the deformation 1-form - as $h$ tends to zero - of any family of Lagrangean submanifolds defined as level sets of functions of the form $p_{i}^{0}+h r_{i}+O\left(h^{2}\right)$.

Of course, the level sets of the total symbols themselves need no be Lagrangean. Recall however that in the case where 0 is a regular value of the principal symbol we can average the sub-principal symbols without changing the cohomology class of $\kappa_{c}$ in such a way that the level sets of $p_{i}^{0}+h r_{i}$ are indeed Lagrangean.

As we shall see in section 5, the Bohr-Sommerfeld quantization conditions that apply to such a situation depend on the sub-principal symbols only through the cohomology class of $\kappa_{c}$. This shows that articles like $[9,5]$ that would rather assume that the sub-principal symbols are either equal to zero or at least constant on each $\Lambda_{c}$ are not far from the general case.

Another nice property of the cohomology class of $\kappa_{c}$ is its invariance under conjugation by unitary pseudo-differential operators.

Proposition 3.3 Let $P_{i}(h)$ be a semi-classical integrable system with a non-singular principal Lagrangean $\Lambda_{0}$. Let $V(h)$ be a classical unitary pseudo-differential operator on a neighborhood of $\Lambda_{0}$. Let $P_{i}^{\prime}=V^{-1} P_{i} V$ be the transformed integrable system and $\kappa^{\prime}$ the new sub-principal form.

Then $\left[\kappa^{\prime}\right]=[\kappa]$.

Proof. Since $P$ and $P^{\prime}$ have same principal symbols, we can write $P^{\prime}=P+h Q$, with $Q=$ $\left(Q_{1}, \ldots, Q_{n}\right)$. The intertwining property $V P^{\prime}=P V$ reads :

$$
\left[P_{j}, V\right]=h V Q_{j}
$$

At the principal level, this gives $\frac{1}{i}\left\{p_{j}, v\right\}=v q_{j}$, which, writing $v=e^{i c}$, yields :

$$
\left\{p_{j}, c\right\}=q_{j}
$$

The sub-principal symbols $r_{j}^{(\prime)}$ of $P^{\left({ }^{\prime}\right)}$ satisfy $r_{j}^{\prime}=r_{j}+q_{j}$, that is,

$$
\kappa^{\prime}=\kappa+d c
$$

where $d c$ denotes the differential of $c$ as a function on $\Lambda_{0}$.

Note that the unitarity of $V$ is only used up to $O\left(h^{2}\right)$ in order to ensure that the sub-principal symbols of the transformed system are real-valued.

\section{Microlocal analysis}

The aim of this section is to present a minimal version of the microlocal analysis needed in order to give a precise and usable definition of what we call "microlocal solutions" of pseudo-differential systems (section 4.5). 
The results can be retrieved from $[11,10,37,30]$; however, note that we focus here a little bit more on the microlocal point of view, and assumptions about "behavior at infinity" are usually irrelevant in this theory.

\subsection{Symbols}

We recall here the basic definitions of symbols and classical symbols. One reason for including here this standard material, besides the sake of completeness, is that our work mainly deals with local properties, in a neighborhood of a point or at least in a neighborhood of a compact region of the phase space; and for this purpose, a very simple notion of 'symbols' can be introduced, which is the following :

Definition 4.1 A family of complex-valued functions $(p(h))_{h \in H}$ on a manifold $Z$, where $H$ is a subset of $\mathbb{R}$ having 0 as an accumulation point, is called a compactly supported symbol if there exists a compact set $K \subset Z$ and an integer $m \in \mathbb{Z}$ such that every $p(h)$ is a $C^{\infty}$ function with support in $K$ such that :

$$
\forall \alpha, \exists C_{\alpha}, \text { s.t. } \forall h \in H, \sup _{z \in Z}\left|\partial_{z}^{\alpha} p(z ; h)\right| \leqslant C_{\alpha} h^{m} .
$$

We denote by $\mathcal{S}_{0}^{m}(Z)$ the vector space of all such symbols. If we drop the condition on the support we obtain the space of all symbols of order $m$, denoted by $\mathcal{S}^{m}(Z)$.

The notation $\mathcal{S}_{(0)}^{m}(Z)$ shall be used in any assertion that holds for $\mathcal{S}_{0}^{m}(Z)$ as well as for $\mathcal{S}^{m}(Z)$.

Remark 4.1. In the notation $\mathcal{S}_{(0)}^{m}(Z)$, we forgot the dependence in $H$. Somewhat later we will have to be more careful, but this omission is harmless at this point.

Note that $\mathcal{S}_{(0)}^{m}(Z) \subset \mathcal{S}_{(0)}^{m^{\prime}}(Z)$ for $m \geqslant m^{\prime}$ and, due to the Leibniz formula, the space $\mathcal{S}_{(0)}^{*}(Z)=$ $\bigcup_{m \in \mathbb{Z}} \mathcal{S}_{(0)}^{m}(Z)$ of symbols of any order is a graded algebra for the usual multiplication. It is also clear that $\mathcal{S}_{(0)}^{m}(Z)=h^{m} \mathcal{S}_{(0)}^{0}(Z)$. If $Z$ sits in a symplectic manifold, we can also endow $\mathcal{S}_{(0)}^{*}(Z)$ with the Poisson bracket $\left\{p_{1}(h), p_{2}(h)\right\}$ turning it into a graded Poisson algebra.

Definition 4.2 A symbol $(p(h))_{h \in H} \in \mathcal{S}_{(0)}^{m}(Z)$ is said to be classical if it admits an asymptotic expansion of the form:

$$
p(z ; h) \sim \sum_{k \geqslant m} p_{k}(z) h^{k}
$$

in the sense that $\forall N \geqslant m, p(h)-\sum_{k=m}^{N} p_{k} h^{k} \in \mathcal{S}_{(0)}^{N+1}(Z)$.

Remark 4.2. If we apply the above definitions to symbols independent of $z$, we get the notion of a "constant symbol". We will let $\mathbb{C}_{h} \hookrightarrow \mathcal{S}^{*}(Z)$ denote the algebra of such constants. With that respect, the spaces $\mathcal{S}^{*}(Z)$ and $\mathcal{S}_{0}^{*}(Z)$ can be considered as modules over $\mathbb{C}_{h}$.

A functional calculus can be performed on symbols of non-negative order; we will only need the following: if $p(h) \in \mathcal{S}_{(0)}^{m}(Z)$ with $m \geqslant 0$, then $\exp p(h) \in \mathcal{S}^{0}(Z)$. Conversely, let $q(h)$ be an element of $\mathcal{S}_{(0)}^{0}(Z)$; if $\Omega$ is any proper simply-connected open subset of $Z$ on which $q(h)$ is 
elliptic (i.e. $|q(h)|>c>0$ on $\Omega$, for all $h$ ), then one can define a symbol $p(h) \in \mathcal{S}_{0}^{0}(Z)$ such that $q(h)=\exp p(h)$ on $\Omega$.

Everything can be restated in the "classical" category.

Definition 4.3 We say that a symbol is $O\left(h^{\infty}\right)$ if it is in $\mathcal{S}^{m}(Z)$ for all $m>0$. We denote by $\mathcal{S}^{\infty}(Z)$ the subspace of all such symbols. Similarly, $\mathcal{S}_{0}^{\infty}(Z)$ is the intersection of all $\mathcal{S}_{0}^{m}(Z)$.

Now, the spaces that will be really of interest for us are the quotient spaces $\mathcal{S}_{(0)}^{*}(Z) / \mathcal{S}_{(0)}^{\infty}(Z)$. Elements in the same class will be called microlocally equal on $Z$. This word is easily justified when $Z$ is a subset of a $T^{*} X$; its extension to any $Z$ should raise no problem. The space of classical symbols modulo microlocal equality can then be isomorphically identified with the space of formal expansions of the form $\sum_{k \geqslant m} p_{k}(z) h^{k}$. Note also that the classical constant symbols modulo microlocal equality form the field of numerical formal series in $h$. In general, as it is easily verified, the quotient of $\mathbb{C}_{h}$ by microlocal equality is a field that we will denote by $\overline{\mathbb{C}}_{h}$.

In order to get meaningful information from the "quantization conditions" that we are going to derive later, we need to consider symbols depending uniformly on a parameter $E \in \mathbb{R}^{n}$. By uniformly, we mean that for every compact subset $B$ of $\mathbb{R}^{n}$, the estimate (1) is valid uniformly for $E \in B$. In the classical case, this is true for instance if each $p_{k}$ depends continuously on $E$.

\subsection{Pseudo-differential operators}

For the general theory, we refer to $[37,10]$. Proofs can usually be derived from the homogeneous theory of Hörmander [31]. We wish here to present a microlocal version of the standard classes of pseudo-differential operators. Let $X$ be a differentiable manifold of dimension $n$, and $M=T^{*} X$. In every local coordinates, a compactly supported pseudo-differential operator $P(h)$ on an open subset $U$ of $X$ is an operator with smooth kernel of the form :

$$
K_{h}(x, y)=\frac{1}{(2 \pi h)^{n}} \int_{\mathbb{R}^{n}} e^{\frac{i}{h}(x-y) \cdot \xi} a(x, y, \xi ; h) d \xi,
$$

where $a(h)$ is a symbol in $\mathcal{S}_{0}^{*}\left(U \times U \times \mathbb{R}^{n}\right)$. Note that $K_{h}$ has compact support in $U \times U$, hence these operators are continuous linear operators (for fixed $h$ ) from $C^{\infty}(U)$ to $C_{0}^{\infty}(U)$, or even from distributions $\mathcal{D}^{\prime}(U)$ to $C_{0}^{\infty}(U)$. We shall assume that a $C^{\infty}$ half density $|d x|^{\frac{1}{2}}$ is given on $X$ so that each function $u$ on $X$ is associated with the half density $u|d x|^{\frac{1}{2}}$.

Pseudo-differential operators have a Weyl symbol, which is a $h$-dependent function $p(h)=$ $\sigma_{W}(P(h))$ on $M$, such that $P(h)$ can be retrieved from $p(h)$ by the so-called "Weyl quantization" scheme :

$$
\left(O p^{W}(p(h)) u\right)(x)=\frac{1}{(2 \pi h)^{n}} \int e^{\frac{i}{h}(x-y) \cdot \xi} p\left(\frac{x+y}{2}, \xi ; h\right) u(y) d y d \xi
$$

Now let $p(h) \in \mathcal{S}_{0}^{m}(\Omega)$, and $\chi \in C_{0}^{\infty}$ equal to 1 in some neighborhood of $0 \in \mathbb{R}^{n}$. We have $a(x, y, \xi ; h)=p\left(\frac{x+y}{2}, \xi ; h\right) \chi(x-y) \in \mathcal{S}_{0}^{m}\left(U \times U \times \mathbb{R}^{n}\right)$, which allows us to form a pseudodifferential operator $O p_{\chi}^{W}(p(h))$ via (2). Then one can show that the Weyl symbol of the latter pseudo-differential operator, although perhaps not of compact support, is nevertheless microlocally equal to $p(h)$ on $\Omega$. That motivates the following definitions : 
Definition 4.4 Let $\Omega$ be an open subset of $M$, in which we have symplectic coordinates $(x, \xi)$. We suppose here that $x$ varies in $U$, an open subset of $X$ containing $\pi(\Omega)$ where $\pi$ is the natural projection $T^{*} X \rightarrow X$. A compactly supported pseudo-differential operator $P(h)$ on $U$ will be called $h$-smoothing or " $O\left(h^{\infty}\right)$ " on $\Omega$ or element of $\Psi_{0}^{\infty}(\Omega)$ if its Weyl symbol belongs to the space $\mathcal{S}^{\infty}(\Omega)$.

The quantization procedure $O p_{\chi}^{W}(p(h))$ is therefore independent of $\chi$ modulo $\Psi_{0}^{\infty}(\Omega)$.

Definition 4.5 We denote by $\Psi_{0}^{m}(\Omega)$ the space of compactly supported pseudo-differential operators of the form $O p_{\chi}^{W}(p(h))+\Psi_{0}^{\infty}(\Omega)$, for any $p(h)$ and $\chi$ defined as above.

Remark 4.3. Dealing with pseudo-differential operators, we shall always assume that the symbols involved are classical.

This definition leads to an isomorphism between the spaces $\Psi_{0}^{m}(\Omega) / \Psi_{0}^{\infty}(\Omega)$ and $\mathcal{S}_{0}^{m}(\Omega) / \mathcal{S}_{0}^{\infty}(\Omega)$ (for the latter is isomorphic to $\frac{\mathcal{S}_{0}^{m}+\mathcal{S}^{\infty}}{\mathcal{S}^{\infty}}$ ). This isomorphism, still denoted by $\sigma_{W}$, depends on the local coordinates, but we recall that the first two terms in the asymptotic expansions of the Weyl symbols are intrinsically defined on $T^{*} X$, provided we let pseudo-differential operators act on half-densities (the proof of this was given in [21, prop 5.2.1] for homogeneous pseudo-differential operators, i.e. without a small parameter $h$, and applies to our situation with no essential change); these two terms are respectively called the principal and sub-principal symbols, and are compactly supported.

It also ensures that theses classes of pseudo-differential operators are stable with respect to the operations of composition and taking adjoints.

We are now able to define the notion of the restriction of a pseudo-differential operator; namely, if $N$ is any subset of $\Omega$, two elements $P(h)$ and $Q(h)$ of $\Psi_{0}^{m}(\Omega)$ will be called microlocally equal on $N$, and written " $P \sim Q$ on $N$ " if $\sigma_{W}(P)=\sigma_{W}(Q)$ on $N$. Then we can perform inversion of elliptic operators in the following sense: $P(h) \in \Psi_{0}^{m}(\Omega)$ is elliptic at a point $m$ if its principal symbol does not vanish at $m$. Then there is a neighborhood $N$ of $m$ and a $Q(h) \in \Psi_{0}^{-m}(\Omega)$ such that $P Q \sim Q P \sim I$ on $N$, where $I$ is a pseudo-differential operator satisfying $I \sim O p^{W}(1)$ on $N$.

Remark 4.4. The space of Weyl symbols is naturally equipped with the Lie algebra structure given by the symplectic Poisson bracket. On the other hand, the space of pseudo-differential operators has a natural Lie operator algebra structure. Though it is known that there is no hope for finding a "quantization" isomorphism that fully respects this Lie algebra structure, the Weyl quantization has the not-so-bad following behavior (see [24]) : Let $p_{1}(h)$ and $p_{2}(h)$ be symbols of order 0 , and $P_{1}(h)$ and $P_{2}(h)$ their Weyl-quantization. Then $\left[P_{1}, P_{2}\right]$ is a pseudo-differential operator of order 1 and of Weyl symbol

$$
\frac{h}{i}\left(\left\{p_{1}, p_{2}\right\}+O\left(h^{2}\right)\right) .
$$

In particular the sub-principal symbol of $\left[P_{1}, P_{2}\right]$ is easily computed in terms of those of $P_{1}$ and $P_{2}$ :

$$
\sigma_{\mathrm{sub}}\left(\frac{i}{h}\left[P_{1}, P_{2}\right]\right)=\left\{p_{1,0}, \sigma_{\mathrm{sub}}\left(P_{2}\right)\right\}+\left\{\sigma_{\mathrm{sub}}\left(P_{1}\right), p_{2,0}\right\}
$$


where $p_{j, 0}$ is the principal symbol of $P_{j}$.

Remark 4.5. The above classes of operators would be purely abstract if we could not relate them to usual $h$-admissible pseudo-differential operators in the sense of [37] and others, for which additional assumptions concerning the behavior of the symbols at infinity are needed. Such a link is here easy: if $P(h) \in \Psi_{0}^{m}(\Omega)$, where $\Omega$ is some open subset of $\mathbb{R}^{n}$, we can form a strongly admissible pseudo-differential operator on $\mathbb{R}^{n}$ by extending the Weyl symbol to zero outside its support, and using a $O p_{\chi}^{W}$ quantization. Conversely, if $Q(h)$ is an admissible pseudo-differential operator in the sense of [37], it has a Weyl symbol, that we can cut to make it compactly supported in $\Omega$, and get a $P(h)$ again. If $Q(h)$ was already obtained by a $P(h)$ in $\Psi_{0}^{m}(\Omega)$, then by definition the new $P(h)$ will microlocally coincide with $P(h)$ on the set where the cut-offs are both equal to 1 . The interest of this microlocalization procedure will be made clear in the next section (prop 4.3).

Finally, compactly supported pseudo-differential operators on manifolds can then be defined to be locally finite sums of operators admitting the above description in a local coordinate chart. The principal and sub-principal symbols remain well-defined functions on $T^{*} X$.

\subsection{Fourier integral operators}

Similarly to the space $\Psi_{0}^{*}(\Omega)$, microlocal classes of Fourier integral operators can be constructed. We will here skip most of the details. Let $X, Y$ be differentiable manifolds, and $\Omega_{X}$ and $\Omega_{Y}$ open subsets of $T^{*} X$ and $T^{*} Y$ respectively. Suppose we have a symplectic diffeomorphism $\chi$ from $\Omega_{Y}$ to $\Omega_{X}$. Let $\Lambda$ be the graph of $\chi$ in $\Omega_{X} \times \Omega_{Y}$. It is an immersed Lagrangean submanifold of $T^{*} X \times \overline{T^{*} Y}$, where $\overline{T^{*} Y}$ denotes the cotangent $T^{*} Y$ equipped with $-\omega$, the opposite of the standard symplectic form. Thus, $\Lambda$ admit parameterization by non-degenerate phase functions $\varphi: \Lambda=\cup \Lambda_{\varphi}$. For details about this construction, refer to [18] or [1]. Following [10], we define $\Psi_{0}^{m}\left(\Omega_{X}, \Omega_{Y}, \chi\right)$ to be the space of operators whose kernel is microlocally equal to a compactly supported classical $h$-oscillatory integral on $\Lambda$ (see paragraph 4.6). On each $\Lambda_{\varphi}$, it has a principal symbol defined as a function on $\Lambda_{\varphi}$. A Fourier integral operator $U(h)$ is said to be elliptic at a point $(x, \xi)$ or, equivalently, at the point $\chi^{-1}(x, \xi)$, if its principal symbol does not vanish at $\left(\chi^{-1}(x, \xi),(x, \xi)\right) \in \Lambda_{\varphi}$.

These operators behave as expected with respect to composition and microlocal inversion of elliptic operators. Most important for us, they allow to find normal forms for pseudo-differential operators thanks to the celebrated theorem of Egorov : if $P(h)$ is a pseudo-differential operator on $\Omega_{X}$ of principal symbol $p$ and $U(h)$ an Fourier integral operator elliptic at a point $(x, \xi)$, then $U^{-1} P U$ is, near $\chi^{-1}(x, \xi)$, a pseudo-differential operator of principal symbol $p \circ \chi$.

\section{$4.4 \quad h$-admissible functionals}

In the above, we have let pseudo-differential operators act on $h$-independent distribution halfdensities, which is of course not enough for our purposes. Let $U$ be a regular domain in $\mathbb{R}^{n}$. If $u \in \mathcal{D}^{\prime}\left(U, \Omega_{\frac{1}{2}}\right)$, then for any pseudo-differential operator $P(h), P u$ is a compactly supported distribution, hence in the Sobolev space $H^{-s}$ for some integer $s$; this holds for any fixed $h$. In order to get asymptotic information for all $h \in H$, we are lead to the following definition (see $[37$, prop IV-8]) : 
Definition 4.6 A family $\left(u_{h}\right)_{h \in H}$ of distribution half densities is called admissible on $\Omega$ if for any pseudo-differential operator $P(h) \in \Psi_{0}^{0}(\Omega)$, there is an $N \in \mathbb{Z}$ and some $s \in \mathbb{Z}$ such that $h^{N}\left(P u_{h}\right)$ is uniformly bounded in $H^{-s}(U)$ for all $h \in H$. We denote by $\mathcal{D}_{h}(\Omega)$ this space of admissible functionals.

$\mathcal{D}_{h}(\Omega)$ is then a $\mathbb{C}_{h}$-module that is by definition stable under the action of $\Psi_{0}^{m}(\Omega)$.

Proposition 4.1 If $u_{h}$ is admissible on $\Omega$ then for any $P(h) \in \Psi_{0}^{0}(\Omega)$ there is an $N^{\prime} \in \mathbb{Z}$ such that $h^{N^{\prime}} P u_{h}$ is bounded in $L^{2}(U)$.

Proof (see [37, p.195-196]). One can find an elliptic operator $D(h)$, that is a zero-order usual pseudo-differential operator on $U$, that uniformly maps $L^{2}(U)$ into some power of $h$ times $H^{-s}(U)$ (extend to $\mathbb{R}^{n}$, and take $D=h^{s}\left(-\Delta+|x|^{2}+1\right)^{s / 2}$ ). Let $D^{-1}$ be a right inverse of $D$, and $P(h) \in \Psi_{0}^{0}(\Omega)$ be as in definition 4.6. So there is a $N^{\prime}$ and a $C<\infty$ such that $\left\|D^{-1}(h) P(h) u_{h}\right\|_{L^{2}(U)}<C h^{-N^{\prime}}$. Now let $\Omega^{\prime} \supset \bar{\Omega}$ and let $I(h) \in \Psi_{0}^{0}\left(\Omega^{\prime}\right)$ have symbol equal to 1 on a neighborhood of $\Omega$. Then $(I(h)-1) P(h) \in \Psi_{0}^{\infty}(\Omega)$, and by the symbolic calculus, $I(h) D(h) \in \Psi_{0}^{0}\left(\Omega^{\prime}\right)$. Therefore, $I(h) D(h)$ is uniformly $L^{2}$-continuous ([37, th.II-36]), and we can write $\left\|P(h) u_{h}\right\|_{L^{2}}=\left\|I(h) D(h) D^{-1}(h) P(h) u_{h}\right\|_{L^{2}}+O\left(h^{\infty}\right) \leqslant C^{\prime} h^{-N^{\prime}}$.

A natural notion of "microlocal equality" for admissible functionals can now be defined :

Definition 4.7 $\mathcal{D}_{h}^{\infty}(\Omega)$ denotes the space of $u_{h} \in \mathcal{D}_{h}(\Omega)$ such that for any pseudo-differential operator $P(h) \in \Psi_{0}^{*}(\Omega)$ we have :

$$
\left\|P u_{h}\right\|_{L^{2}(U)}=O\left(h^{\infty}\right) .
$$

Two admissible functionals $u_{h}$ and $v_{h}$ are called microlocally equal on $\Omega$ if they belong to the same class modulo $\mathcal{D}_{h}^{\infty}(\Omega)$. Following [11], we will write " $u_{h} \sim v_{h}$ on $\Omega$ " in that case. If $m \in T^{*} X$, we say that $u_{h} \sim v_{h}$ at the point $m$ if there exists an open neighborhood $\Omega$ of $m$ such that $u_{h} \sim v_{h}$ on $\Omega$.

Note that, because the above definition only involves estimates on compact subsets of $\Omega, u_{h} \sim v_{h}$ on $\Omega$ if and only if $u_{h} \sim v_{h}$ at each point of $\Omega$. Moreover, to test microlocal equality at a point, it is sufficient to pick up an elliptic operator that satisfies the required estimate. There again we have obvious although useful properties :

Proposition 4.2 - if $P(h) \in \Psi_{0}^{\infty}(\Omega)$, then for all $u_{h} \in \mathcal{D}_{h}(\Omega), P u_{h} \sim 0$ on $\Omega$;

- if $u_{h} \sim 0$ on $\Omega$, then for all $P(h) \in \Psi_{0}^{*}(\Omega), P u_{h} \sim 0$ on $\Omega$.

This is essentially due to the $L^{2}$-continuity of pseudo-differential operator of order 0 . In other words, the quotient space $\Psi_{0}^{*}(\Omega) / \Psi_{0}^{\infty}(\Omega)$ acts naturally on $\mathcal{D}_{h}(\Omega) / \mathcal{D}_{h}^{\infty}(\Omega)$. This says that the action of a pseudo-differential operator on an admissible functional is microlocally given by the (formal) Weyl symbol $\sigma_{W}$. Note also that the space $\mathcal{D}_{h}(\Omega) / \mathcal{D}_{h}^{\infty}(\Omega)$ has the structure of a $\overline{\mathbb{C}}_{h}$-vector space.

In particular, if $N$ is any subset of $\Omega$, the definition 4.7 gives a natural microlocal equality on $N$, which is compatible with the notion of restriction of a pseudo-differential operator previously introduced: two pseudo-differential operators microlocally equal on $N$ have the same action on two admissible functionals microlocally equal on $N$.

The same remark applies for the following interesting result : 
Proposition 4.3 If $Q(h)$ is a global admissible pseudo-differential operator in $\mathbb{R}^{n}$ in the sense of [37], $\Omega$ an open subset of $\mathbb{R}^{2 n}, u_{h}$ an admissible functional in $\Omega$, and $P(h) \in \Psi_{0}^{*}(\Omega)$ a microlocalization of $Q$ in a compact $K$ of $\Omega$, then $Q u_{h}$ is admissible in $\Omega$ and:

$$
Q u_{h} \sim P u_{h} \text { on } K \text {. }
$$

Alternatively, one can relate this notion of microlocal equality for admissible functionals to the so-called semi-classical wave front set, as introduced in [37, 10], and denoted by $W F_{h}\left(u_{h}\right)$. This notion is not stabilized yet, in the sense that, depending on authors, it includes or not uniform estimates at infinity (see remark in [11, p.1541]). Anyhow, the simpler part of $W F_{h}$, that is to say its intersection with $T^{*} X$, is defined as follows :

suppose $u_{h}$ is admissible on $T^{*} X$. Then $W F_{h}\left(u_{h}\right) \cap T^{*} X$ is the complement subset in $T^{*} X$ of the biggest open subset $\Omega$ such that $u_{h} \sim 0$ on $\Omega$.

The definition is of course based upon the homogeneous wave front set introduced by Hörmander (see [30, sec.2.5]). It has a useful local characterization in terms of the semi-classical Fourier transform :

Lemma 4.4 ([37, 10]) An admissible functional $u_{h}$ microlocally vanishes at the point $\left(x_{o}, \xi_{o}\right) \in$ $T^{*} X$ (equivalently, $\left.\left(x_{o}, \xi_{0}\right) \notin W F_{h}\left(u_{h}\right)\right)$ if and only if there exists a function $\varphi \in C_{0}^{\infty}(X)$ with $\varphi\left(x_{o}\right) \neq 0$ such that, in some local coordinates :

$$
\mathcal{F}_{h}\left(\varphi u_{h}\right)(\xi)=O\left(h^{\infty}\right)
$$

uniformly for $\xi$ in a neighborhood of $\xi_{0}$.

Here $\mathcal{F}_{h}$ denotes the "semi-classical Fourier transform" :

$$
\mathcal{F}_{h}=\frac{1}{(2 \pi h)^{\frac{n}{2}}} \int_{\mathbb{R}^{n}} e^{-\frac{i}{h} x \cdot \xi} d x
$$

(we will sometimes use the usual Fourier transform $\mathcal{F}=\mathcal{F}_{1}$ ). Note that $\mathcal{F}_{h}$ is a priori defined for tempered distributions, but the Fourier transform of a compactly supported distribution in $\mathbb{R}^{n}$ can be thought as a continuous function (it is analytic). For the proof of this lemma, one constructs a compactly supported pseudo-differential operator with "rectangular support", that is with symbol of the form $\chi(\xi) \varphi(x)$.

Unfortunately, because of the lack of uniform estimates in the fibers one should not expect an analogue of theorem 2.5.3 of the previously cited article [30]. Indeed, if $U$ is an open set of $X$, then $W F_{h}\left(u_{h}\right) \cap T^{*} U=\emptyset$ does not imply that $u_{h}$ is locally $O\left(h^{\infty}\right)$ on $U$. For instance, one easily sees from lemma 4.4 that $u_{h}(x)=a(x) e^{i x / h^{2}}$ is microlocally zero on $T^{*} \mathbb{R}^{n}$, while obviously not locally $O\left(h^{\infty}\right)$.

The solution found in [10] for functionals on $\mathbb{R}^{n}$ is to extend the wave front set to a subset of the compactified cotangent bundle obtained by adding a point at infinity to each direction $\xi$. An admissible functional $u_{h}$ is then said to be microlocally zero in the direction $\left(x_{0}, \mathbb{R}^{+} \xi_{0}\right)$ if, for large $\xi$ in a conic neighborhood of $\xi_{0}$, and for any cut-off function $\varphi$, the Fourier transform :

$$
\mathcal{F}_{h}\left(\varphi u_{h}\right)(\xi)
$$

is of order $O\left(h^{N} /|\xi|^{N}\right)$ for all $N>0$. Then the following statement is valid : 
Lemma 4.5 An admissible functional $u_{h}$ is $O\left(h^{\infty}\right)$ uniformly for $x$ near $x_{o}$ if and only if there exists a function $\varphi \in C_{0}^{\infty}(X)$ with $\varphi\left(x_{o}\right) \neq 0$ such that :

$$
\mathcal{F}_{h}\left(\varphi u_{h}\right)(\xi)=O\left(h^{N} /(1+|\xi|)^{N}\right)
$$

for all $\xi \in \mathbb{R}^{n}$, for all $N \in \mathbb{N}$.

The condition expressed by this lemma is that there exists a neighborhood $U$ of $x_{o}$ such that $u_{h}$ is microlocally zero in $T^{*} U$ as well as in all directions of $T^{*} U$.

Similarly to lemma 4.4, we have for instance the following fact : let $u_{h}$ be an admissible functional defined on $\mathbb{R}^{n}$, so that $\mathcal{F}_{h} u_{h}$ makes sense. If $\mathcal{F}_{h} u_{h}$ is a $O\left(h^{\infty}\right)$ function in a neighborhood of any $\xi$ in an open set $\Upsilon$, then $u_{h}$ is microlocally zero in the whole $\mathbb{R}^{n} \times \Upsilon$. This allows the following construction : let $1=\chi_{1}+\cdots+\chi_{k}$ be a partition of unity subordinated to the open cover $\bigcup_{j} \Upsilon_{j}$ in the $\xi$-space. Let $U$ be an open subset of $\mathbb{R}^{n}$ with compact closure. If $v_{j, h}, j=1, \ldots, k$ are admissible functionals on $U \times \Upsilon_{j}$ that microlocally coincide on non-empty intersections $U \times \Upsilon_{j_{1}} \cap \cdots \cap \Upsilon_{j_{l}}$, one can define an admissible functional $u_{h}$ on $U \times \bigcup_{j} \Upsilon_{j}$ by cutting off the $v_{j, h}$ 's outside $\bar{U}$ in $\mathbb{R}^{n}$ and requiring :

$$
\mathcal{F}_{h} u_{h}=\sum_{j=1}^{k} \chi_{j} \mathcal{F}_{h} v_{j} .
$$

Then $u_{h}$ microlocally coincides with each $v_{j, h}$ on $U \times \Upsilon_{j}$. Indeed, from the microlocal equality of the $v_{j, h}$ 's on non-empty intersections, and the compactness of their support, it follows from lemma 4.4 that the Fourier transforms $\mathcal{F}_{h} v_{j, h}$ are $C^{\infty}$ functions that coincide with each other up to $O\left(h^{\infty}\right)$ around each point of such non-empty intersections. Thus $\mathcal{F}_{h} u_{h}$ also has that common value around these points, which yields the result.

Finally, if $U(h) \in \Psi_{0}^{m}\left(\Omega_{X}, \Omega_{Y}, \chi\right)$, then for any admissible $u_{h}$ in $\Omega_{Y}, U u_{h}$ is admissible in $\Omega_{X}$, and because of Egorov's theorem, $U$ transports the wave front set by $\chi$.

\subsection{Microlocal solutions}

Definition 4.8 Let $\Omega$ be an open set in $T^{*} X$. An admissible functional $u_{h} \in \mathcal{D}_{h}(\Omega)$ is called a microlocal solution on $\Omega$ of a system $\left(P_{1}(h), \ldots, P_{k}(h)\right)$ of pseudo-differential operators if :

$$
\forall j=1, \ldots, k, \quad P_{j} u_{h} \sim 0 \text { on } \Omega .
$$

We allow here to choose a suitable set $H$ where $h$ is to vary. The space of all microlocal solutions of $\left(P_{1}, \ldots, P_{k}\right)$ modulo microlocal equality will be viewed as a $\overline{\mathbb{C}}_{h}$-vector space. Because of microlocal inversion of elliptic operators, any such solution is "microlocalized" in $\bigcap_{j} p_{j}^{-1}(0)$, which means that $W F_{h}\left(u_{h}\right)$ must lie in this set $\left(p_{j}\right.$ denotes the principal symbol of $\left.P_{j}\right)$. If two solutions have different non-empty wave front sets, then they are independent. In case the $p_{j}$ 's are real-valued, the "propagation of singularities" theorem states that $W F_{h}\left(u_{h}\right)$ is locally stable under the action of the Hamiltonian flow of $p_{j}$.

As we will see later, global problems often have no solution in the previous sense. Suppose that the $P_{j}$ depend continuously on an additional parameter $E$ in a compact topological space 
$V$ (cf. end of section 4.1). Depending on the degeneracy of the $p_{j}$ 's, it might be impossible to find a solution (in the previous sense) for some $E$, and depending on $E$, the appropriate sets $H_{E}$ where $h$ varies might be disjoint. Still, the whole theory being asymptotic, we need to control things when $h$ tends to zero! Therefore, we introduce the following :

Definition 4.9 Let $\Gamma$ be a subset of $] 0,1] \times V$. Let $H$ be the projection of $\Gamma$ onto $] 0,1]$. We assume that 0 is an accumulation point of $H$. We say that a family $\left(u_{(h, E)}\right)_{(h, E) \in \Gamma}$ is admissible if definition 4.6 holds uniformly for $(h, E) \in \Gamma$.

Recall that $E$ varies in a compact. Accordingly, $u_{(h, E)}$ microlocally vanishes at point $m$ if there is an elliptic pseudo-differential operator $P(h)$ at $m$ such that $\left\|P u_{(h, E)}\right\|_{L^{2}(U)}=O\left(h^{\infty}\right)$ uniformly for $(h, E) \in \Gamma$. Thus we are still able to define a semi-classical wave-front set $W F_{h}\left(u_{(h, E)}\right)$.

Now if $u_{(h, E)}$ is admissible and $P_{j}^{E}$ depend continuously on $E$, then $P_{j}^{E} u_{(h, E)}$ is admissible, and we say that $u_{(h, E)}$ is a microlocal solution of the system $\left(P_{1}^{E}, \ldots, P_{k}^{E}\right)$ on $\Omega$ if $P_{j}^{E} u_{(h, E)} \sim 0$ on $\Omega$ according to that definition. Then we have :

Proposition 4.6 Let $u_{(h, E)}$ be a microlocal solution of the equation $P^{E} u_{(h, E)} \sim 0$ on $\Omega$. The following inclusion holds :

$$
W F_{h}\left(u_{(h, E)}\right) \subset\left\{(x, \xi) \in T^{*} X, \quad \exists E_{o} \in V_{o}, \quad p_{E_{o}}(x, \xi)=0\right\},
$$

where $V_{o}$ is the set of $E_{o} \in V$ such that $\left(0, E_{o}\right)$ is an accumulation point of $\Gamma$. $p_{E}$ is the principal symbol of $P^{E}(h)$.

Proof. Let $(x, \xi) \in T^{*} X$ such that for any $E_{o} \in V_{o}$, the principal symbol $p_{E_{o}}$ is non zero at $(x, \xi)$. Since $V_{o}$ is compact and $p_{E}$ is continuous in $E$, then there is an open neighborhood $\mathcal{V}$ of $V_{o}$ and a constant $c>0$ such that for $E \in \mathcal{V},\left|p_{E}\right|>c$. Then there is an open neighborhood $\Omega$ of $(x, \xi)$ on which that remains valid. The complement $\mathcal{V}^{c}$ being a compact set of non-accumulation points, we can pick up a $h_{o}$ such that $\left(\left[0, h_{o}\right] \times \mathcal{V}^{c}\right) \cap \Gamma$ is empty. From now on, we will therefore restrict $\Gamma$ to $\left[0, h_{o}\right] \times \mathcal{V}$. Because of the uniform ellipticity of $P^{E}$ on $\Omega$, for $(h, E)$ in that new $\Gamma$, we can construct a pseudo-differential operator $Q^{E}(h)$ depending continuously on $E \in \mathcal{V}$ such that $Q^{E} P^{E} \sim I$ on $\Omega$. It is not difficult to see that if an admissible $v_{(h, E)}$ is microlocally zero at a point $m$, then for any pseudo-differential operator $Q^{E}(h)$ depending continuously on $E$ in some compact, then $Q^{E} v_{(h, E)}$ is still microlocally zero. Back in our problem, we see that :

$$
u_{(h, E)} \sim 0 \text { at }(x, \xi)
$$

which is what we needed to prove.

\subsection{Oscillatory integrals}

The WKB ansatz for solving Schrödinger-type equations consists of restricting the scope of solutions to a subspace of admissible functionals : the space of oscillatory integrals, that we wish to describe now. These functions have a long history, particularly in Quantum mechanics, and it is well known since the treatise of Maslov [34], made more rigorous and expounded by Hörmander and Duistermaat (see in particular [18]), that they are particularly fit to locally solve 
generic microlocal pseudo-differential equations. In fact, as we shall see later, in some cases all solutions are microlocally equal to oscillatory integrals.

We recall here the definitions of [18], with some precisions.

We are given an compact immersed Lagrangean manifold $\Lambda \in T^{*} X$, endowed with a smooth half-density $\rho$. Suppose that is fixed a covering of $\Lambda$ with simply connected embedded open subsets $\Lambda_{\varphi_{k}}$ described by reduced phase functions $\varphi_{k}(x, \theta)$ ("reduced" means that the number of additional oscillatory variables $\theta$ is exactly the maximum dimension of the kernel of $d \pi$, where $\pi$ is the projection $\Lambda_{\varphi} \rightarrow X$. This dimension is known to be the minimum dimension of $\theta$ variables needed for a phase function defining $\Lambda-[30$, theo 3.1.4] or $[18,1.3 .6]) . \varphi$ gives rise to a function on $\Lambda_{\varphi}$ - that we still denote by $\varphi$ - whose exterior differential is the Liouville 1 -form $\alpha_{0}$.

An oscillatory symbol on $\Lambda_{\varphi}$ is a half-density $\sigma(h)$ on $\Lambda_{\varphi}$ of the form :

$$
\sigma(\lambda ; h)=c(h) e^{\frac{i}{h} \varphi(\lambda)} a(\lambda ; h) \rho(\lambda),
$$

with $c(h) \in \mathbb{C}_{h}$, and $a(h)$ a classical symbol in $\mathcal{S}^{*}\left(\Lambda_{\varphi}\right)$. Will will say that $\sigma$ is classical if $c$ is.

If $a(h)$ is of order 0 and elliptic on $\Lambda_{\varphi}$ (as we will generally assume), we will prefer the notation :

$$
\sigma(\lambda ; h)=e^{i c(h)+i \Phi(\lambda ; h)} \rho(\lambda),
$$

where $c(h) \in \mathbb{C}_{h}$, and $\Phi(h)$ is a $\mathcal{S}^{1}$-valued classical symbol on $\Lambda_{\varphi}$ of order -1, whose principal symbol $\frac{\Phi-1}{h}$ satisfies :

$$
d \Phi_{-1}=\alpha_{0}
$$

As usual, we denote by $j_{\varphi}$ the local diffeomorphism from $C_{\varphi}$ to $\Lambda_{\varphi}$ given by $(x, \theta) \mapsto$ $\left(x, \frac{\partial \varphi}{\partial x}(x, \theta)\right)$, where $C_{\varphi}=\left\{(x, \theta) \in X \times R^{N}, \frac{\partial \varphi}{\partial \theta}(x, \theta)=0\right\}$.

An oscillatory integral on $\Lambda_{\varphi}$ is a half-density on $X$ of the form :

$$
u_{h}(x)=\frac{c(h)}{(2 i \pi h)^{\frac{N}{2}}} \int_{\mathbb{R}^{N}} e^{\frac{i}{h} \varphi(x, \theta)} b(x, \theta ; h) d \theta|d x|^{\frac{1}{2}},
$$

where $c(h) \in \mathbb{C}_{h}$, and $b(h)$ is a classical symbol in $\mathcal{S}_{0}^{*}\left(X \times \mathbb{R}^{N}\right)$. Then

$$
\sigma=\left(j_{\varphi}\right)^{-1 *}\left(c e^{\frac{i}{h} \varphi} b_{\mid C_{\varphi}}\right)
$$

is an oscillating symbol. $u_{h}$ is an admissible functional, and because of the stationary phase formula, is uniquely defined modulo microlocal equality by the asymptotic expansion of $b(h)$ on any neighborhood of $C_{\varphi} . \sigma$ is thus well defined by $u_{h}$ modulo microlocal equality.

Definition 4.10 An admissible functional $u_{h}$ is a Lagrangean distribution on $\Lambda$ if there is a cover $\Lambda=\cup \Lambda_{\varphi}$ such that $u_{h}$ is microlocally equal to an oscillatory integral on each $\Lambda_{\varphi}$.

On each $\Lambda_{\varphi}$, such Lagrangean distributions have a symbol $\sigma(h)$ defined modulo $O\left(h^{\infty}\right)$, but in general $(N \neq 0)$ the morphism $u_{h} \mapsto \sigma(h)$ is not injective; however, one can prove that it is an isomorphism at the principal level (if $u_{h}$ can be written with $b(h)$ of order $m$ such that the 
$h^{m}$ part of $\sigma(h)$ vanishes on a neighborhood of $\left(x_{o}, \xi_{o}\right) \in \Lambda_{\varphi}$, then near $j_{\varphi}^{-1}\left(x_{o}, \xi_{o}\right), u_{h}$ can be rewritten with a $b(h)^{\prime}$ of order $\left.m+1\right)$.

On the other way round, the symbol map is surjective, and one can construct right inverses ("Maslov canonical operators") in the following way : let $\sigma(h)$ be a oscillatory symbol near $\left(x_{o}, \xi_{o}\right) \in \Lambda_{\varphi}$. Then it defines the function $b(h)$ on a neighborhood of $b_{o}=j_{\varphi}^{-1}\left(x_{o}, \xi_{o}\right)$ in $C_{\varphi}$. If $N>0, C_{\varphi}$ is a proper submanifold of $X \times \mathbb{R}^{N}$. Let $B_{o}$ be a sub-bundle of $T\left(X \times \mathbb{R}^{N}\right)$ supplementary to $T C_{\varphi}$ near $b_{o}$. One defines the germs of $b$ on $C_{\varphi}$ to be zero along $B_{o}$, and smoothly extends $b$ to $X \times \mathbb{R}^{N}$ accordingly. Then $b$ has the same $h$-order as $\sigma(h)$ and the oscillatory integral $u_{h}=O p(\sigma)$ so constructed has symbol $\sigma(h)\left(\bmod O\left(h^{\infty}\right)\right)$.

Of course, since we have not made any mention to the Keller-Maslov bundle yet, the symbol of a Lagrangean distribution depends on the phase function $\varphi$ considered. The result of HörmanderMaslov is the following: if $u_{h}$ and $v_{h}$ are zeroth order oscillatory integrals on $\Lambda_{\varphi}$ and $\Lambda_{\psi}$ respectively that are equal modulo $O(h)$ at a point $(x, \xi) \in \Lambda_{\varphi} \cap \Lambda_{\psi}$, then there is an integer $\mu$ such that, at the principal level, $\sigma_{u_{h}} \sim e^{i \mu \frac{\pi}{2}} \sigma_{v_{h}}+O(h)$ at $(x, \xi)$. Because of the normalization in (6), $\mu=\mu_{\psi}-\mu_{\varphi}$, where, for a phase function $g, 2 \mu_{g}$ is the signature of the $\theta$-Hessian of $g$ at $(x, \xi)$ minus $N_{g}$.

With these transition functions, one can globally define the principal symbol of a Lagrangean distribution as a section of the Keller-Maslov bundle. In the sequel, Maslov indices will always be prompted very clearly, so we don't insist any further on that point.

Note that if $u_{h}$ serves to construct a Fourier integral operator $U(h)$ as mentioned in section 4.3, then the principal symbol of $u_{h}$ is invariantly defined by $U$.

If the symbol is elliptic and we write it as (5), we will refer to $\Phi(h)$ as the total phase of $u_{h}$, and the principal phase is $\frac{\Phi_{-1}}{h}+\Phi_{0}$, both defined modulo a constant $c(h) \in \mathbb{C}_{h}$.

Finally, we admit the dependence of a Lagrangean distribution on an additional parameter $E$, provided the involved estimates are uniform in the sense of definition 4.9. It is the case for instance if the symbol $b$ depends continuously on $E$.

\section{$5 \quad$ Non-singular quantization conditions}

Let $P_{1}(h), \ldots, P_{n}(h)$ be a semi-classical integrable system, and denote by $\Lambda_{0}$ the principal Lagrangean manifold associated to it. $\Lambda_{0}$ may be critical, but in this section we shall work on a submanifold $\Lambda \subset \Lambda_{0}$ consisting only of non-singular points of the momentum mapping $\left(p_{1}, \ldots, p_{n}\right)$. Moreover, we shall always assume that $\bar{\Lambda}$ is compact.

With these data, we express a necessary and sufficient condition for the existence of microlocal solutions of the system on $\Lambda$ (proposition 5.6). Thanks to the WKB construction, we prove that this condition involves, at least at the principal level, geometrical characteristics of the system, namely the Liouville 1 -form $\alpha_{0}$ and the sub-principal form $\kappa$ (theorem 5.8).

As an application, we recover the well-known Maslov-Bohr-Sommerfeld quantization conditions for $O\left(h^{2}\right)$ solutions in the case where $\Lambda=\Lambda_{0}$ is a whole non-singular Liouville torus.

When the system depends smoothly on an $n$-dimensional parameter $E$ in a suitable sense, the above results are seen to be uniform on $E$, which will allow us to apply the quantization condition to the study of semi-classical spectra. In the case of a non-singular Liouville torus, the study of the spectrum was also completely carried out, with a different approach, in [5]. 


\subsection{Dimension of microlocal solutions}

The following result is a generalization of lemma 18 of [11].

Proposition 5.1 Let $x \in \Lambda$ be a non-singular point of $\Lambda_{0}$. The space of microlocal solutions of the system $P_{i} u \sim 0$ on a neighborhood of $x$, modulo microlocal equality, has dimension 1.

If $x \notin \Lambda_{0}$, the space of solutions of this system has dimension 0.

Proof. The second point comes from the fact that near $x$, the system is elliptic, hence microlocally invertible (see proposition 4.6).

Concerning the first one, we use the following microlocal normal form, which is standard :

Lemma 5.2 There exists an elliptic Fourier integral operator $U(h)$ associated to a DarbouxCarathéodory coordinate chart $(x, \xi)$ such that

$$
U^{-1} P_{j} U \sim \frac{h}{i} \frac{\partial}{\partial x_{j}}
$$

on a neighborhood of $x$. If every $P_{j}$ is formally self-adjoint, then $U$ can be chosen to be unitary $\left(\bmod O\left(h^{\infty}\right)\right)$.

Accordingly transformed, the system always admits the constant solutions. It remains now to prove that these are the only ones, that is, that any solution $u_{h}$ of $\frac{\partial}{\partial x_{j}} u \sim 0$ is microlocally constant. Let $u_{h}$ be such a solution. We can always assume that it is compactly supported. We want now to "cut-off in the frequencies", which we do in the following way :

Let $v_{h}$ an admissible functional defined by $\mathcal{F}_{h} v_{h}=\chi \mathcal{F}_{h} u_{h}$, where the cut-off function $\chi$ is $C_{0}^{\infty}$ and has value 1 on a neighborhood of 0 . Then $u_{h}$ and $v_{h}$ are microlocally equal on a neighborhood of 0 . Moreover, $v_{h}$ is a local solution (modulo $O\left(h^{\infty}\right)$ ) of the system near 0 . This comes from the fact that $\mathcal{F}_{h}\left(\frac{h}{i} \frac{\partial v_{h}}{\partial x_{j}}\right)=\xi_{j} \mathcal{F}_{h} v_{h}$ satisfies the hypothesis of lemma 4.5.

We have thus reduced the problem to a system of the form :

$$
d_{h} v_{h}=w_{h}=O\left(h^{\infty}\right)
$$

on a neighborhood of 0 (here $d_{h}$ denotes the exterior differential operator $d_{h}=\left(\frac{h}{i} \frac{\partial}{\partial x_{1}}, \ldots, \frac{h}{i} \frac{\partial}{\partial x_{n}}\right)$ ). The solutions of this system are necessarily of the form

$$
\operatorname{cste}_{h}+\frac{i}{h} \int_{0}^{1} w_{h}(t x) \cdot x d t
$$

The second term in the sum is $O\left(h^{\infty}\right)$, as $w_{h}$ was. Hence it is microlocally zero, which gives the result.

Let $\varphi_{t}=\varphi_{1}\left(t_{1}\right) \circ \cdots \circ \varphi_{n}\left(t_{n}\right)$ be the classical flow associated to the momentum map of the system. By transport of singularities, we obtain the following corollary :

Corollary 5.3 If the action of $\varphi_{t}$ on $\Lambda$ is transitive, then the space of microlocal solutions of the system $P_{i} u \sim 0$ on $\Lambda$ has dimension at most 1 . 
If we restrict to a simply connected open subset $\Omega$ of $\Lambda_{0}$, and if all trajectories of the flow remain open (which is automatic if $\Omega$ contains no singular point), then local microlocal solutions can be glued together to obtain a non-trivial solution of the system on $\Omega$. This occurs for instance if $\Omega$ is obtained by propagating a non-singular point of $\Lambda_{0}$ by the flow during a short time (cf. theorem 1.4.1 of [18]).

As soon as we have closed trajectories, we get obstructions, which we describe in the following paragraph. Later on (paragraph (5.4)), we will show how to interpret these obstructions thanks to the WKB method, and get the so-called "Bohr-Sommerfeld quantization conditions".

\subsection{The bundle of microlocal solutions}

For $x \in \Lambda$, let $\mathcal{L}_{h}(x)$ be the 1-dimensional vector space over $\overline{\mathbb{C}}_{h}$ of microlocal solutions of the system at the point $x$ (modulo microlocal equality). For any open subset $\Omega$ of $\Lambda$, the disjoint union $\mathcal{L}_{h}(\Omega)$ of the $\mathcal{L}_{h}(x), x \in \Omega$ has a natural structure of a $\overline{\mathbb{C}}_{h}$-line bundle over $\Omega$, defined by the following local trivializations : if $\Omega=\cup \Omega_{\alpha}$, where every $\Omega_{\alpha}$ is chosen small enough so that the system admits a non-trivial microlocal solution $u_{h}^{\alpha}$ on it, then we define $\varphi_{\Omega_{\alpha}}$ to be the isomorphism :

$$
\begin{gathered}
\varphi_{\Omega_{\alpha}}: \Omega \times \overline{\mathbb{C}}_{h} \rightarrow \mathcal{L}_{h}(\Omega) \\
(x, c(h)) \mapsto c(h) u_{h}^{\alpha} .
\end{gathered}
$$

If we restrict to non-trivial solutions, this also defines a principal bundle $\dot{\mathcal{L}}_{h}(\Lambda)$ over $\Omega$ with structure group $\overline{\mathbb{C}}_{h}{ }^{*}=\overline{\mathbb{C}}_{h} \backslash\{\overline{0}\}$. This bundle has a natural flat connection, whose parallel sections are those that are locally constant in the above trivializations (from the infinitesimal viewpoint, its horizontal distribution is defined to be the image of the standard horizontal spaces of $\Omega \times \overline{\mathbb{C}}_{h}$ by the trivialization maps $\left.\varphi_{\Omega_{\alpha}}\right)$. It is well defined because on intersections $\Omega_{\alpha} \cap \Omega_{\beta}$, such trivializations differ by a constant, due to proposition 5.1. By the same argument, it is also independent of the set of microlocal solutions $u_{h}^{\alpha}$ chosen to define the trivializations. The interest of this connection is stressed by the following lemma :

Lemma 5.4 There is a natural identification of the space of non-trivial microlocal solutions of the system on an open subset $\Omega \subset \Lambda$ with parallel sections of $\dot{\mathcal{L}}_{h}(\Omega)$.

Proof. Let us first consider the case $\Omega=\Omega_{\alpha}$. The general case will follow by a microlocal partition of unity. Now, using the trivialization $\varphi_{\Omega_{\alpha}}$, a section of $\mathcal{L}_{h}\left(\Omega_{\alpha}\right)$ takes the form :

$$
\sigma_{h}(x)=c(x ; h) u_{h}^{\alpha} .
$$

This section is parallel if and only if $c(x ; h)=c(h)$ is a constant (modulo microlocal equality, of course), so, by proposition 5.1, if and only if $\sigma_{h}(x)=\sigma_{h}$ is a microlocal solution on $\Omega_{\alpha}$. It is non-trivial if and only if $c_{h} \neq 0$.

Now assume $\Omega$ is any open subset of $\Lambda$. One sense is obvious : if $u_{h}$ is a non-trivial solution on $\Omega$, then it can be used to trivialize the line bundle $\mathcal{L}_{h}(\Omega)$ as in $(7)$. Now, since the definition of the connection is independent of the chosen solutions, the constant section $\sigma_{h}(x)=u_{h}$ is a parallel section. 
On the other way round, suppose $\sigma_{h}(x)$ is a parallel section of $\dot{\mathcal{L}}_{h}(\Omega)$. Then we proved that its restrictions to each $\Omega_{\alpha}$ are non-trivial microlocal solutions $v_{h}^{\alpha}$ on $\Omega_{\alpha}$. Let $P^{\alpha}(h)$ be a pseudo-differential partition of unity on $\Omega$ subordinated to the cover $\Omega_{\alpha}$ (it means that the Weyl symbols form a $C_{0}^{\infty}$ partition of unity of $\Omega$ ). Then each $P^{\alpha} v_{h}^{\alpha}$ is an admissible functional on a neighborhood of $\Omega$, and the sum

$$
v_{h}=\sum_{\alpha} P^{\alpha} v_{h}^{\alpha}
$$

is microlocally equal to $v_{h}^{\alpha}$ (and hence to $\sigma_{h}$ ) on each $\Omega_{\alpha}$. Therefore, $v_{h}$ is a microlocal solution on $\Omega$, whose associated parallel section is $\sigma_{h}$. This terminates the proof of the lemma.

Thus, the obstruction to the existence of a microlocal solution on $\Lambda$ only lies in the holonomy of the connection, which, in this case, describes the monodromy of the solution. This holonomy can be represented by the cohomology class of a Čech 1 -cocycle on $\Lambda$ with values in $\overline{\mathbb{C}}_{h}{ }^{*}$. Summarizing, we get :

Proposition 5.5 There exists a non-trivial microlocal solution of the system on $\Lambda$ if and only if the holonomy cocycle of the $\overline{\mathbb{C}}_{h}{ }^{*}$-principal bundle $\dot{\mathcal{L}}_{h}(\Lambda)$ with its natural flat connection has microlocally trivial cohomology.

From now on, our principal task will be to explicit that holonomy. Besides the above definition, we can give a direct and more concrete description of it in terms of Cech cohomology, that shall be more efficient for our further analysis.

We still let $\Omega_{\alpha}$ be a (finite) cover of $\Lambda$ by simply connected open subsets such that each $\Omega_{\alpha}$ admits a neighborhood in $M$ on which proposition 5.1 applies. We thus get a family $u_{h}^{\alpha}$ of non-trivial microlocal solutions of the system on $\Omega_{\alpha}$. Moreover, on non-empty intersections $\Omega_{\alpha} \cap \Omega_{\beta}$, the same proposition affirms the existence of unique non-zero constants $c^{\beta \alpha}(h) \in \mathbb{C}_{h}^{*}$ such that:

$$
u_{h}^{\beta} \sim c^{\beta \alpha} u_{h}^{\alpha} \text { on } \Omega_{\alpha} \cap \Omega_{\beta} .
$$

The $c^{\beta \alpha}$ 's satisfy the cocycle condition :

$$
c^{\beta \alpha} \sim c^{\beta \gamma} c^{\gamma \alpha}
$$

on every non-empty intersection $\Omega_{\alpha} \cap \Omega_{\beta} \cap \Omega_{\gamma}$. Furthermore, another choice of solutions $u_{h}^{\prime \alpha}$ would give rise to constants $c^{\prime \beta \alpha}$ differing by a coboundary $d^{\alpha}(h)$ :

$$
c^{\prime \beta \alpha} \sim \frac{d^{\beta}}{d^{\alpha}} c^{\beta \alpha}
$$

This Cech cocycle $c^{\beta \alpha}$ is exactly the holonomy form of the connection introduced above. The matching conditions are then the following :

"there exists a non-trivial microlocal solution on $\Lambda$ if and only if $c^{\beta \alpha}(h)$ is a coboundary (modulo $\left.O\left(h^{\infty}\right)\right)$."

It is, of course, a simple re-formulation of proposition 5.5. In the sequel, instead of the complex-valued Cech cocycle $[c(h)]$, we will rather deal with the two real-valued cocycles obtained via the exponential representation :

$$
c^{\beta \alpha}=e^{\rho^{\beta \alpha}} e^{i \lambda^{\beta \alpha}},
$$


in which $\rho^{\beta \alpha}(h) \in \mathbb{R}$ and $\lambda^{\beta \alpha}(h) \in \mathbb{T}$. We denote by $\rho(h)$ and $\lambda(h)$ the corresponding Cech cocycles.

Proposition 5.6 Suppose that every $P_{j}$ is formally self-adjoint. Then $[\rho(h)]$ is microlocally trivial, and $[\lambda(h)]$ admits an asymptotic expansion of the form :

$$
[\lambda(h)] \sim \sum_{k \geqslant-1}\left[\lambda_{k}\right] h^{k}
$$

Proof. We cover $\Lambda$ by open subsets $\Omega_{\alpha}$ on which the normal form of lemma 5.2 applies. This gives rise to a family of microlocally unitary Fourier integral operators $U^{\alpha}(h)$ on $\Omega_{\alpha}$. An associated set of solutions is given by $u_{h}^{\alpha}=U^{\alpha} 1_{\alpha}$, where $1_{\alpha}$ denotes an admissible functional microlocally equal to the constant 1 on $\Omega_{\alpha}$. Suppose $\Omega_{\alpha} \cap \Omega_{\beta}$ is a non-empty intersection and let $x$ be an element of it. Finally, let $1_{x}$ denote an admissible functional microlocally equal to 1 on a neighborhood of $x$. On $x$, we have $u_{h}^{\alpha} \sim U^{\alpha} 1_{x}$ and $u_{h}^{\beta} \sim U^{\beta} 1_{x}$. Therefore, the constant $c^{\beta \alpha}(h)$ is given by :

$$
c^{\beta \alpha} 1_{x} \sim\left(U^{\alpha}\right)^{-1} U^{\beta} 1_{x}
$$

The unitarity of the Fourier integral operators implies that $\left|c^{\beta \alpha}\right| \sim 1$, which gives the first statement of the proposition.

Now, $\left(U^{\alpha}\right)^{-1} U^{\beta}$ is an elliptic Fourier integral operator which implies that $\left(U^{\alpha}\right)^{-1} U^{\beta} 1_{x}$ is at $x$ a classical Lagrangean distribution with a symbol of the form (5). This gives the last statement of the proposition.

Remark 5.1. The description of the cocycle $c^{\beta \alpha}(h)$ involves no regularity on $\Lambda$. And indeed, the notion of a flat line bundle with holonomy is well-defined on any topological manifold. This will enable us, in section 6.7, to apply that description on a singular Lagrangean. For the moment however, the $C^{\infty}$ structure of $\Lambda$ makes it possible to apply the De Rham isomorphism and get a smooth connection 1-form $\lambda(h)$ on $\Lambda$. In the next sections, precisely, we show how the 1 -forms $\alpha_{0}$ and $\kappa$ enter the picture.

\subsection{WKB method}

Although this might not seem obvious, propositions 5.5 and 5.6 are essentially related to the celebrated WKB construction.

The WKB method for solving pseudo-differential equations consists in looking for a special kind of approximate solutions, called oscillatory integrals (see section 4.6). The use of semiclassical oscillatory integral in mathematics was initiated by Maslov, and pursued by different authors including Arnold, Hörmander and Duistermaat. The result is that, provided we are given an invariant half-density on $\Lambda$ (this will come from the Liouville measure), we can always construct such solutions in a neighborhood of a non-singular point of $\Lambda_{0}$. According to proposition 5.1, such a solution has to be the only one - up to multiplicative constant. This means that we can freely use the WKB construction to look for general properties of our microlocal integrable systems. This is the goal of the following paragraph. 
Note that we previously constructed microlocal solutions via another method, namely the use of a normal form. The link with oscillatory integrals lies in the use of a Fourier integral operator; more precisely, with the notations of lemma 5.2, the admissible functional $U 1$ is a Lagrangean distribution that is a microlocal solution of the system on a neighborhood of $x$ (1 denotes a functional microlocally equal to the constant 1 on a neighborhood of $x$ ).

The derivation of the transport equations in the WKB method for completely integrable systems is fairly standard now (see for instance [8]), and our aim here is only to briefly recall the idea. Of particular importance is the entrance of the sub-principal form $\kappa$ into the picture.

Let $u_{h}$ be a Lagrangean distribution on $\Lambda_{0}$ of order 0 . To ensure that it is not trivial, we assume that it is elliptic at a point $\left(x_{0}, \xi_{0}\right) \in \Lambda_{0}$, so that we use the notation (5) for the symbol $\sigma(h)$ (we assume that we work on a fixed $\Lambda_{\varphi}$ ). The viewpoint here is to find conditions on $\sigma$ in order that $u_{h}$ be a microlocal solution of the completely integrable system at $\left(x_{0}, \xi_{0}\right)$.

First of all, if one looks for a solution up to $O(h)$ terms, one finds the condition that $\Phi_{-1}$ is indeed a phase function for $\Lambda_{\varphi}$, i.e. : on $\Lambda_{\varphi}$,

$$
\frac{d \Phi_{-1}}{h}=\frac{\alpha_{0}}{h}
$$

Note that this first condition only involves the principal symbols of the $P_{j}$ 's.

Keeping $\Phi_{-1}$ fixed, one can look now for a solution up to $O\left(h^{2}\right)$. Since we suppose that the sub-principal symbols are real (which occurs for instance if the $P_{j}$ 's are formally self-adjoint), then this raises two necessary and sufficient conditions : a) the half-density $\rho$ must be invariant with respect to the Hamiltonian action of the system; b) on $\Lambda_{\varphi}$, one must have

$$
d \Phi_{0}=\kappa,
$$

where $\kappa$ is the sub-principal form of the system. Because of condition a), we can assume that $\rho$ is the canonical Liouville half-density on $\Lambda_{0}$. Condition b) implies that $\kappa$ must be closed, which is already given by the fact that the $P_{j}$ 's commute with each other up to the order $O\left(h^{2}\right)$. Finally, note that if the sub-principal symbols are complex-valued, the conditions are still sufficient, but not necessary.

The previous step generalizes by induction to provide solutions up to any order. However, notice that the two conditions obtained above depend only on the principal symbol of $u_{h}$ and on some closed 1-forms uniquely defined by the system. The next conditions involving higher order terms of the symbol won't of course be invariantly defined anymore. Still, there is a way to keep track of the total symbol of $u_{h}$, and this is to fix a "Canonical operator" (see section 4.6) $u_{h}=O p(\sigma(h))$, as stated in the following proposition :

Proposition 5.7 Let $\Lambda_{\varphi}$ be a neighborhood of $\left(x_{0}, \xi_{0}\right)$ in $\Lambda_{0}$ described by a phase function $\varphi$. Let $O p$ be a Canonical operator on $\Lambda_{\varphi}$. There exists closed real-valued 1 -forms $\kappa_{k}, k=$ $-1,0,1, \ldots, \infty$ on $\Lambda_{0}$ such that :

The oscillatory integral $u_{h}=O p\left(e^{i \Phi(\lambda ; h)} \rho(\lambda)\right)$ is a microlocal solution of the system up to the order $O\left(h^{N}\right)$ on $\Lambda_{\varphi}$ if and only if the $\Phi_{k}$ 's, $k=-1, \ldots, N-2$ satisfy :

$$
d \Phi_{k}=\kappa_{k} .
$$

$\kappa_{-1}$ is the Liouville form $\alpha_{0}$ restricted to $\Lambda_{0}$, and $\kappa_{0}$ is the sub-principal form $\kappa$. For $k>0$, $\kappa_{k}$ depends on the Op-quantization. 
A similar result holds if the $P_{j}$ 's depend continuously on a parameter $E$. Note that in [8, p.38] Y.Colin de Verdière included the dependence on a parameter directly in the definition of the oscillatory symbols.

\subsection{The regular quantization conditions}

Theorem 5.8 We still assume that the $P_{j}$ 's are formally self-adjoint, and that $\Lambda$ is a regular open subset of $\Lambda_{0}$.

Let $[\lambda(h)] \in H^{1}(\Lambda, \mathbb{T})$ be, as in proposition 5.6, the holonomy phase of the microlocal bundle $\dot{\mathcal{L}}_{h}(\Lambda)$. There exists a non-trivial microlocal solution of the system on $\Lambda$ if and only if

$$
[\lambda(h)] \in H^{1}(\Lambda, 2 \pi \mathbb{Z})+O\left(h^{\infty}\right),
$$

and any other solution is then a multiple of it. Moreover, $[\lambda]$ admits the following expansion :

$$
[\lambda(h)] \sim[\mu] \frac{\pi}{2}+\sum_{k=-1}^{\infty}\left[\lambda_{k}\right] h^{k},
$$

where $\mu$ is the Maslov cocycle, $\lambda_{-1}$ is the restriction of Liouville 1-form $\alpha_{0}$ on $\Lambda$, and $\lambda_{0}=\kappa_{\uparrow \Lambda}$ is the sub-principal form of the system.

Proof. The first points are direct consequences of propositions 5.5, 5.6 and 5.1. It remains to prove the statement about the principal terms of the asymptotic expansion of $[\lambda]$. We cover $\Lambda$ by simply connected open subsets $\Omega_{\alpha}$ such that each $\Omega_{\alpha}$ admits a representation by a non degenerate phase function $\varphi_{\alpha}$. The most immediate consequence of proposition 5.7 is that microlocal solutions as Lagrangean distributions always exist on $\Omega_{\alpha}$; Let us denote by $u_{h}^{\alpha}$ such a set of solutions, and by $\Phi^{\alpha}(h)$ their principal phases. Finally, we let $\mu^{\alpha \beta}$ be the (integral) value of Maslov's cocycle associated with that cover.

The equation

$$
u_{h}^{\beta} \sim c^{\beta \alpha}(h) u_{h}^{\alpha} \text { on } \Omega_{\alpha} \cap \Omega_{\beta}
$$

implies that $\arg c^{\beta \alpha}$ is, mod $O(h)$, the difference of the principal phases of $u_{\psi}$ and $u_{\varphi}$, plus the value of the corresponding Maslov cocycle. That is,

$$
\arg c^{\beta \alpha}(h)=\Phi^{\alpha}(h)-\Phi^{\beta}(h)+\mu^{\alpha \beta} \frac{\pi}{2}+O(h) .
$$

Because of proposition 5.7, $\Phi^{\alpha}-\Phi^{\beta}$ is the value of a Čech cocycle whose cohomology is represented, via the de Rham isomorphism, by the cohomology of the smooth 1-form $\frac{\alpha_{0}}{h}+\kappa$ restricted to $\Lambda$. Therefore, $\arg c^{\beta \alpha}$ is represented, modulo $O(h)$, by $\frac{\left[\alpha_{0}\right]}{h}+[\kappa]+[\mu] \frac{\pi}{2}$. Since $\arg c^{\beta \alpha}$ is itself a representative of $[\lambda]$, the theorem is proved.

If $\Lambda=\Lambda_{0}$ is a whole Liouville torus, the principal terms in the quantization condition yield the well-known Maslov-Bohr-Sommerfeld quantization condition : "For all closed paths $\gamma$ in $\Lambda_{0}$ (or equivalently for the $n$ standard generators of $H_{1}\left(\Lambda_{0}\right)$ obtained through action-angle coordinates),

$$
\frac{1}{h} \int_{\gamma} \alpha_{0}+\int_{\gamma} \kappa+\mu(\gamma) \frac{\pi}{2} \in 2 \pi \mathbb{Z}+O(h) " .
$$




\subsection{Spectral parameter dependence}

The motivation of this paragraph is to make the above results suitable for studying spectral problems, namely for investigating the solutions of the microlocal system :

$$
\left(P_{1}(h)-E_{1}\right) u_{h} \sim 0, \ldots,\left(P_{n}(h)-E_{n}\right) u_{h} \sim 0,
$$

according to the appropriate definition of microlocal equality (section 4.5).

In order to do this, the operators $P_{j}$ in the quantization conditions have to be replaced by $P_{j}-E_{j}$. In the following analysis, we will need to state the problem in a slightly more general setting, where we replace $P_{j}$ by an operator depending on $E$ in essentially the same way as $P_{j}-E_{j}:$

Definition 5.1 Let $P_{i}(h)$ be a semi-classical integrable system on an n-dimensional manifold $X$, with principal symbols $p_{i}$. A system $P_{i}^{E}(h)$ depending smoothly on the parameter $E \in \mathbb{R}^{n}$ will be called regular deformation of $P_{i}(h)$ if for each $E,\left(P_{1}^{E}(h), \ldots, P_{j}^{E}(h)\right)$ is a semi-classical integrable system, with principal symbols $p_{i}^{E}$, and there exists a $C^{\infty}$ function $f^{E}(x)=f(E, x)$, $f: \mathbb{R}^{n} \times \mathbb{R}^{n} \rightarrow \mathbb{R}^{n}$ such that $f^{0}=i d$ and $f(\cdot, 0)$ is a local diffeomorphism of $\left(\mathbb{R}^{n}, 0\right)$, such that:

$$
\left(p_{1}^{E}, \ldots, p_{n}^{E}\right)=f^{E}\left(p_{1}, \ldots, p_{n}\right) .
$$

For such a regular deformation, the new Lagrangean foliation $\Lambda_{c}^{E}$ is globally the same as the original one $\Lambda_{c}$, with different labels : $\Lambda_{c}=\Lambda_{f E(c)}^{E}$.

Now we assume that there is a tubular neighborhood $\Omega$ of $\Lambda$ such that for $E$ in a sufficiently small compact neighborhood of 0 , the intersections $\Lambda^{E}=\Lambda_{0}^{E} \cap \Omega$ are non-singular submanifolds of $\Lambda_{0}^{E}$. Then it is easy to verify that every result of this section extends to uniform solutions of the perturbed system $P_{i}^{E}(h)$ on $\Omega$. In particular we obtain a perturbed cocycle $\left[c^{E}(h)\right]$ that smoothly depends on $E$. It implies that the terms in the asymptotic expansion in powers of $h$ of the cocycle $\left[\lambda^{E}(h)\right]$, equal to the argument of $\left[c^{E}(h)\right]$, are locally smooth but possibly multivalued functions of $E$. At the principal levels, this gives a parameter dependent version of the Bohr-Sommerfeld conditions (8) : "for any family of loops $\gamma^{E}$ drawn on $\Lambda_{0}^{E}$,

$$
\frac{1}{h} \int_{\gamma^{E}} \alpha_{0}+\int_{\gamma^{E}} \kappa^{E}+\mu\left(\gamma^{E}\right) \frac{\pi}{2} \in 2 \pi \mathbb{Z}+O(h)^{\prime} .
$$

Remark 5.2. Let $P_{i}^{E}(h)$ be such a smooth deformation. Then one can look at the family obtained by letting $E=h t$, where $t$ is a bounded parameter (let's assume for clarity in this remark that $n=1$; everything applies to the many-dimensional case as well). Using that in every local coordinates, the Weyl symbols of $P_{I}^{E}$ is a smooth function of $E$, one can see that the operator

$$
\tilde{P}^{t}(h) \stackrel{\text { def }}{=} P^{h t}(h)
$$

is still a classical pseudo-differential operator, with uniform estimates for bounded $t$, but its principal and sub-principal symbols are of course not the same as those of $P^{E}(h)$. More precisely, if we denote by $r^{E}$ the sub-principal symbol of $P^{E}$, then, because of Taylor's formula :

$$
f(E, x)=f(0, x)+E \frac{\partial f}{\partial E}(0, x)+\cdots+E^{N} \frac{\partial^{N} f}{\partial E^{N}}(0, x)+O\left(E^{N+1}\right),
$$


which holds uniformly for bounded $E$, the principal and sub-principal symbols $\tilde{p}^{t}$ and $\tilde{r}^{t}$ of $\tilde{P}^{t}(h)$ satisfy :

$$
\tilde{p}^{t}=p^{0}=f^{0}(p), \quad \text { and } \quad \tilde{r}^{t}=r^{0}+t{\frac{\partial f^{E}}{\partial E}\lceil E=0}(p) .
$$

Now, let us see what Bohr-Sommerfeld conditions (9) become. First of all, (11) shows that the principal Lagrangean $\tilde{\Lambda}_{0}$ of the new system is independent of $t$ and equal to $\Lambda_{0}^{0}$. Let us call $\tilde{\kappa}^{t}$ the sub-principal form on $\Lambda_{0}^{0}$. On the other hand, the family of Lagrangean manifolds $\Lambda_{0}^{E}=\left\{f^{E}(p)=0\right\}$, with $E=h t$, depends smoothly on $h$; let us call $\delta \kappa^{t}$ its deformation 1-form at $h=0$ (see 3.2.2), defined by :

$$
\delta \kappa^{t} \cdot \mathcal{X}_{f^{0}(p)}=-t{\frac{\partial f^{E}}{\partial E}\lceil E=0}(p) .
$$

Then paragraph 3.2.4 together with (11) shows that, on $\Lambda_{0}^{0}$,

$$
\tilde{\kappa}^{t}=\kappa^{0}+\delta \kappa^{t} .
$$

Now, if $\gamma^{E}$ is a smooth family of loops drawn on $\Lambda_{0}^{E}$, lemma 3.2 implies that, when $E=h t$,

$$
\int_{\gamma^{E}} \alpha_{0}=\int_{\gamma^{0}} \alpha_{0}+h \int_{\gamma^{0}} \delta \kappa^{t}+O\left(h^{2}\right) .
$$

Since it is clear that $\int_{\gamma^{E}} \kappa^{E}=\int_{\gamma^{0}} \kappa^{0}+O(h)$, we get :

$$
\frac{1}{h} \int_{\gamma^{E}} \alpha_{0}+\int_{\gamma^{E}} \kappa^{E}=\frac{1}{h} \int_{\gamma^{0}} \alpha_{0}+\int_{\gamma^{0}} \tilde{\kappa}^{t}+O(h) .
$$

In other words, we exactly recover the Bohr-Sommerfeld quantization conditions we would have expected to find for the $t$-dependent system $\tilde{P}^{t}(h)$.

Note that this remark will mostly be used with $f(E, x)=x-E$, so that $\tilde{r}^{t}=r^{0}-t$.

\section{Bohr-Sommerfeld for a focus-focus singularity}

\subsection{Integrable systems with a non-degenerate singularity}

Let $M$ be any symplectic manifold of dimension $2 n$, and $f_{1}, \ldots, f_{n}$ a completely integrable system on $M$. The system is said to be singular at a point $m \in M$ if $m$ is a critical point for the momentum map $F$. We shall always suppose in this work that $m$ is of maximal corank as a critical point, which means that each function $f_{i}$ is critical at $m$. Without loss of generality, we shall also assume that for all $i=1, \ldots, n, f_{i}(m)=0$.

The map $\mathcal{H}$ that assigns to a smooth function its Hessian at $m$ is a Lie algebra homomorphism with respect to the Poisson bracket from the Lie algebra of smooth functions that are critical at $m$ to the Lie algebra of quadratic forms on the tangent space $T_{m} M$. Fixing any local set of Darboux coordinates, the latter is identified with the space $\mathcal{Q}(2 n)$ of quadratic forms on $\mathbb{R}^{2 n}=\{(x, \xi)\}$.

Every singular completely integrable system thus gives rise to a linear sub-algebra $C_{F}$ of $\mathcal{Q}(2 n)$, namely the sub-algebra generated by $\left\{\mathcal{H}\left(f_{1}\right), \ldots, \mathcal{H}\left(f_{n}\right)\right\}$. Note that $C_{F}$ is always Abelian. 
Definition 6.1 (Eliasson [23]) A singular completely integrable system with momentum map $F$ admits $m$ as a non-degenerate singularity if $C_{F}$ is a Cartan sub-algebra of $\mathcal{Q}(2 n)$.

Recall that a Cartan subalgebra of a semi-simple Lie algebra $\mathfrak{a}$ is a commutative subalgebra $\mathfrak{c}$ that is equal to its commutant and such that, for any element $H \in \mathfrak{c}, a d_{H}$ is a semi-simple endomorphism of $\mathfrak{a}$.

From the work of Williamson [43], real Cartan sub-algebras of $\mathcal{Q}(2 n)$ are classified according to the following scheme :

Theorem 6.1 (Williamson) Let $\mathfrak{c}$ be a real Cartan sub-algebra of $\mathcal{Q}(2 n)$. Then there exists a symplectic linear change of coordinates in $\mathbb{R}^{2 n}$ and a basis $q_{1}, \ldots, q_{n}$ of $\mathfrak{c}$ such that each $q_{i}$ is one of the following :

- $q_{i}=x_{i}^{2}+\xi_{i}^{2}$ (elliptic type)

- $q_{i}=x_{i} \xi_{i}$ (hyperbolic type)

- $\left.\begin{array}{c}q_{i}=x_{i} \xi_{i}+x_{i+1} \xi_{i+1} \\ q_{i+1}=x_{i} \xi_{i+1}-x_{i+1} \xi_{i}\end{array}\right\}$ (focus-focus type)

If $M$ has dimension 4, as we shall assume from now on, only four combinations are possible. We will restrict ourselves to the case of a focus-focus singularity. Note that this is the only case where $m$ is isolated amongst the set of critical points of $F$. In particular, the singular foliation $\Lambda_{c}=F^{-1}(c)$ has, for small $c$, only one singular leaf $\Lambda_{0}$, and if $\Omega$ is a sufficiently small neighborhood of $m$ in $M$, then $\Omega \backslash\{m\}$ is foliated by smooth Lagrangean submanifolds of $M$. If $\Lambda_{0}$ is connected, this also implies that the critical value 0 lies in the interior of the image of the momentum map $F$. The aim of the following paragraphs is to give a precise description of the singular fibration on a full neighborhood of $\Lambda_{0}$, generalizing the usual Arnold-Liouville theory for regular fibers. The results are not new, since they are quoted in [35] and proposition 6.2 can in principle be recovered from [32], but I believe that the following presentation gives a clearer account of what is needed for our purposes.

\subsection{Linear focus-focus}

We fix here the notations used when referring to a linear focus-focus system on $\mathbb{R}^{4}=\{(x, y, \xi, \eta)\}$. We shall always use Williamson coordinates, in which the system is generated by the quadratic forms $q_{1}=x \xi+y \eta$ and $q_{2}=x \eta-y \xi$. The Hamiltonian flows are denoted by $\mathcal{X}_{1}$ and $\mathcal{X}_{2}$. It will be convenient to use polar coordinates $(r, \theta)$ for $(x, y)$ and $(\rho, \alpha)$ for $(\xi, \eta)$, in which $\mathcal{X}_{1}$ and $\mathcal{X}_{2}$ take a simpler form (see figure 1). Of interest are also the complex representations $z_{1}=x+i y$ and $z_{2}=\xi+i \eta$. The flows of $\mathcal{X}_{1}$ and $\mathcal{X}_{2}$ are then respectively

$$
\begin{gathered}
\left(t,\left(z_{1}, z_{2}\right)\right) \mapsto\left(e^{t} z_{1}, e^{-t} z_{2}\right), \text { and } \\
\left(s,\left(z_{1}, z_{2}\right)\right) \mapsto\left(e^{i s} z_{1}, e^{i s} z_{2}\right) .
\end{gathered}
$$

Similarly, we can identify the momentum map $F$ with the complex-valued function $F=q_{1}+i q_{2}$, which gives

$$
F\left(z_{1}, z_{2}\right)=\overline{z_{1}} z_{2}
$$

The singular leaf $\Lambda_{0}=F^{-1}(0)$ is the union of the coordinate planes $P_{s}=\left\{z_{1}=0\right\}$ and $P_{u}=\left\{z_{2}=0\right\}$, which are respectively the stable and unstable manifolds for the $\mathcal{X}_{1}$-flow. 


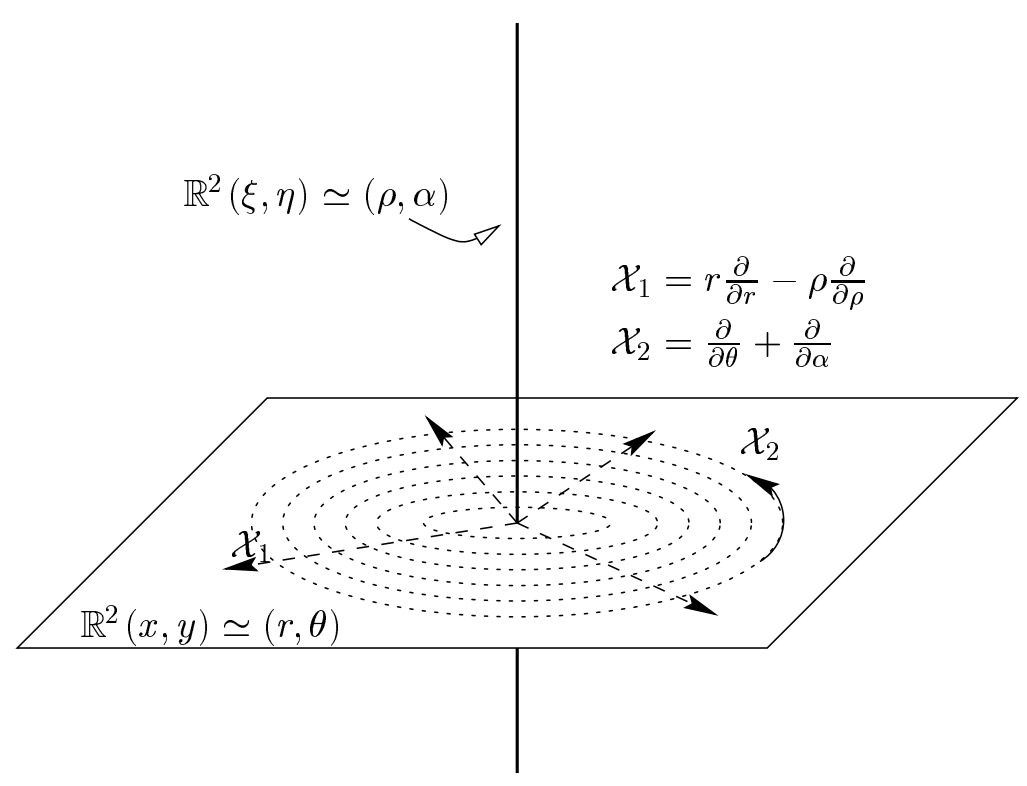

Figure 1: the linearized flow

\subsection{Geometry of the singular Lagrangean}

Singular points of focus-focus type are isolated, but it may occur that the singular Lagrangean $\Lambda_{0}$ contains several of them. Since $\Lambda_{0}$ is assumed to be compact, there will only be a finite number of them. However, we will always assume that $\Lambda_{0}$ carries only one critical point, which is certainly a "generic" assumption (in [35], it is claimed that one can always reduce the number of focus-focus points by a small perturbation of the momentum map). This point will always be denoted by $m$.

Recall that the usual theory of Arnold-Liouville gives action-angle coordinates in a neighborhood of any connected compact regular leaf. Here, we have compactness, but without smoothness. Another viewpoint would be to consider the non-compact punctured leaf $\Lambda_{0} \backslash\{m\}$; it is smooth, and invariant under the action of the joint Hamiltonian flow of the system (this holds because the critical point $\{m\}$ is a fixed point). But there is no hope for the neighboring leaves to be diffeomorphic to $\Lambda_{0} \backslash\{m\}$, since they are compact...

Nevertheless, the local structure of $\Lambda_{0}$ near the singularity turns out to be sufficient data for its global description.

Proposition 6.2 Let $\Lambda_{0}$ be a singular leaf of the momentum map, carrying a unique critical point $m$ of focus-focus type. Then the connected component of $m$ in $\Lambda_{0}$ is the image of a Lagrangean immersion of a 2-sphere with a double point. Deprived of $m$, it is an orbit of the Hamiltonian action of the system, with the structure of an affine infinite cylinder.

Proof. First of all, note that we can suppose that $\Lambda_{0}$ is connected. Indeed, this won't lead to any loss of information, since any other connected components are necessarily regular tori, for which the usual theory applies. 
On $\Lambda_{0} \backslash\{m\}$, the action is locally free, hence by standard arguments each connected component of $\Lambda_{0} \backslash\{m\}$ (there are at most two of them) is an orbit, on which all isotropy subgroups $I$ are conjugated. Therefore, this orbit is diffeomorphic to $\mathbb{R}^{2} / I$, which can be either $\mathbb{R}^{2}$, or the infinite cylinder $\mathbb{R}^{2} / \mathbb{Z} \simeq S^{1} \times \mathbb{R}$ (it cannot be a torus because it is not compact).

The local structure of the flow makes up for this ambiguity. Indeed, according to Eliasson's theorem [22], the action is symplectically linearizable near $m$ : there exists a symplectic chart in which the Hamiltonian vector fields of $f_{1}$ and $f_{2}$ are linear combinations of the standard focusfocus vector fields; moreover, the involved coefficients form an invertible $2 \times 2$ matrix $M_{c}$ which is locally constant along each fiber $\Lambda_{c}$. Now, the focus-focus vector fields are by assumption associated to the Hamiltonians $q_{1}$ and $q_{2}$. Since $q_{2}$ has periodic orbits in any neighborhood of $m$, the isotropy subgroup $I$ is necessarily isomorphic to $\mathbb{Z}$. The connected components of $\Lambda_{0} \backslash\{m\}$ are therefore infinite cylinders.

On such a cylinder are globally defined two infinitesimal generators of the action, $\mathcal{X}_{1}$ and $\mathcal{X}_{2}$, by the requirement that they are obtained from the initial generators $\mathcal{X}_{f_{1}}$ and $\mathcal{X}_{f_{2}}$ by applying the matrix $M_{c}^{-1}$. In Eliasson's coordinates, these vector fields are of course the standard focusfocus generators. The first one $\mathcal{X}_{1}$ is always transversal to $\mathcal{X}_{2}$, and hence describes an "axis" of the cylinder. $\mathcal{X}_{1}$ and $\mathcal{X}_{2}$ define the affine structure of the cylinder.

Let us show now that $\Lambda_{0} \backslash\{m\}$ is necessarily connected. From the connectedness of $\Lambda_{0}$ and the local structure at the critical point $m$, we a priori know that $\Lambda_{0} \backslash\{m\}$ has at most two connected components, which correspond respectively to the stable and unstable manifolds of $\mathcal{X}_{1}$. Let $x$ be a point on the unstable manifold, contained in a small $S^{1}$-invariant neighborhood $U$ of $m$. We let the flow of $\mathcal{X}_{1}$ act on $x$ so that when time increases, the image $x(t)$ of $x$ goes out of $U$. Now, we know from the dynamic on the infinite cylinder that $x(t)$ must go out of any compact subset in finite time. Since the manifold with boundary $\Lambda_{0} \backslash U$ is compact, the image of $x$ must necessarily enter $U$ again. But it is clear from the local structure of the flow that $x(t)$ can approach $m$ only via the stable manifold. Therefore, the stable and the unstable manifold are connected to each other, and hence they are equal.

Thus, $\Lambda_{0}$ is homeomorphic to a cylinder whose extremities are compactified in a unique point $m$, or a "pinched torus", or a sphere with two points identified.

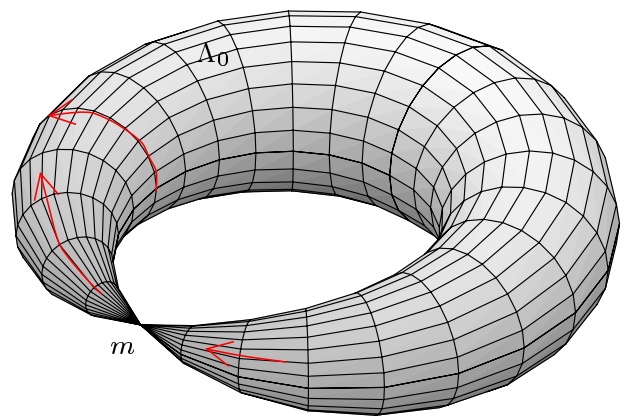

Figure 2: topology of $\Lambda_{0}$

Remark 6.1. We can readily deduce that $\pi_{1}\left(\Lambda_{0}\right)$ is isomorphic to $\mathbb{Z}$, the integer coefficient counting the winding number along the "big axis of the pinched torus". 
From the differentiable viewpoint, we see in the local form that $\Lambda_{0} \cap U$ is the union of two smooth submanifolds diffeomorphic to open discs $D^{2}$ and transversally intersecting at $m$. Therefore $\Lambda_{0}$ is the immersion of a smooth 2-manifold with a unique double point. Since this manifold can be obtained by gluing a $D^{2}$ at each extremity of the cylinder $\Lambda_{0} \backslash U$, it is a sphere.

\subsection{Monodromy of the Lagrangean fibration around $\Lambda_{0}$}

The first description of the monodromy invariant for singular fibrations was carried out in [19], where the example of the spherical pendulum was studied. Later, Maorong Zou in [44] and Nguyên Tiên Zung in [35] observed that the monodromy at stake was typical of a focusfocus singularity. In [35], the computation of the monodromy is based on a claimed normal form for the foliation. We present here another proof of the result, relying on the local but Hamiltonian normal form of Eliasson. The constructions explained in the proof will be used for the quantization conditions (sections 6.6 and 6.7).

At the same time as I was writing this article, Duistermaat and Cushman were able to generalize the monodromy for non-Hamiltonian systems [16].

Let us first briefly recall the definition, from a topological viewpoint. For any completely integrable system in dimension $2 n$, a regular value $c$ of the momentum map $F$ gives rise to a Liouville torus $\Lambda_{c}$, and thus to a lattice $L_{c}=H_{1}\left(\Lambda_{c}, \mathbb{Z}\right)$. Because $F$ is a smooth fibration near $F^{-1}(c)$, the union of all $L_{c}$ is a locally trivial smooth bundle $L$ over the open set $D_{r}$ of regular values of $F$. Fixing a point $c_{0}$ and a smooth basis of $H_{1}\left(\Lambda_{c}\right) \simeq \mathbb{Z}^{n}$, a local section of $L$ near $c_{0}$ takes values in $\mathbb{Z}^{n}$, and thus is locally constant. We therefore have the notion of parallel transport above a path in $D_{r}$. The holonomy associated with it is a homomorphism from $\pi_{1}\left(D_{r}, c_{0}\right)$ to $\operatorname{Aut}\left(L_{c_{0}}\right) \simeq \mathrm{GL}(n, \mathbb{Z})$, called the monodromy of $L$.

Back to our focus-focus system, we take $D_{r}$ to be a small ball around the origin in $\mathbb{R}^{2} \simeq \mathbb{C}$, minus the origin. Because its $\pi_{1}$ is $\mathbb{Z}$, the problem is reduced to determining the monodromy of a simple loop around 0 .

Recall from last paragraph that for any focus-focus system $\left(p_{1}, p_{2}\right)$, there is a local normal form $\left(q_{1}, q_{2}\right)=f\left(p_{1}, p_{2}\right)$ near $m$, where $f$ is a local diffeomorphism of $\left(\mathbb{R}^{2}, 0\right)$, and $q_{i}$ 's are defined in symplectic coordinates around $m$ by paragraph 6.2. This yields two particular vector fields $\mathcal{X}_{1}$ and $\mathcal{X}_{2}$ defined on a full neighborhood of $\Lambda_{0}$ by :

$$
\left(\mathcal{X}_{1}, \mathcal{X}_{2}\right)=d f .\left(\mathcal{X}_{p_{1}}, \mathcal{X}_{p_{2}}\right)
$$

The flow of $\mathcal{X}_{2}$ on $\Lambda_{c}$ is, as we already saw, periodic of period $2 \pi$, so the orbit of any point $x$ is a simple loop $\Gamma_{x}$ on $\Lambda_{c}$ depending smoothly on $x$. Its homology class $\gamma_{2}\left(c_{0}\right)$ is therefore an invariant of the monodromy operator. Let us denote by $\gamma_{1}\left(c_{0}\right)$ the homology class of a path starting on $\Gamma_{x}$ in the direction of $\mathcal{X}_{1}$, going back to $\Gamma_{x}$ and closing up on $\Gamma_{x}$ in the direction of $\mathcal{X}_{2}$.

$\left(\gamma_{1}\left(c_{0}\right), \gamma_{2}\left(c_{0}\right)\right)$ is a basis of $L_{c_{0}}$ depending locally smoothly on $c_{0}$; the monodromy of the system is expressed on this basis by the following proposition :

Proposition 6.3 ([35]) Let $\left(p_{1}, p_{2}\right)$ be a completely integrable system with a singular leaf carrying a unique singular point of focus-focus type. Then for any $c_{0}$ close enough the the critical 
value $0 \in \mathbb{R}^{2}$, the monodromy matrix, expressed in the basis $\left(\gamma_{1}\left(c_{0}\right), \gamma_{2}\left(c_{0}\right)\right)$ of $L_{c_{0}}$, is equal to

$$
\left(\begin{array}{ll}
1 & 0 \\
\epsilon & 1
\end{array}\right)
$$

Here $\epsilon$ is the sign of $\operatorname{det} M$, where $M \in G L(2, \mathbb{R})$ is the unique matrix such that :

$$
\left(\mathcal{H}\left(q_{1}\right), \mathcal{H}\left(q_{2}\right)\right)=M \cdot\left(\mathcal{H}\left(p_{1}\right), \mathcal{H}\left(p_{2}\right)\right) .
$$

Proof. The idea of the proof, already apparent in the construction of the basis $\left(\gamma_{1}\left(c_{0}\right), \gamma_{2}\left(c_{0}\right)\right)$, is to show that all contributions to the monodromy can be concentrated in a small neighborhood of the critical point $m$.

Let $U$ be such a small ball around $m$, taken to be $S^{1}$-invariant. The intersection of $\partial U$ with $\Lambda_{0}$ is composed of two circles $\Gamma_{u}$ and $\Gamma_{s}$ respectively drawn on the local unstable and stable manifolds at $m$. Let us take a point $x$ on $\Gamma_{u}$, and let $\Sigma$ be a small section transversal to $\Lambda_{0}$ at $x$. The tangent space to $\Lambda_{0}$ at $x$ is exactly the kernel of $d F$. Thus, the momentum map $F$ realizes a local diffeomorphism from $(\Sigma, x)$ onto $\left(\mathbb{R}^{2}, 0\right)$. We can then assume that $\Sigma$ is a preimage of a small open disc $D \subset D_{r}$ around 0 .

Let $\Omega$ be the invariant open subset of $M$ obtained as the union of orbits of points in $\Sigma$. It is a neighborhood of $\Lambda_{0}$, composed of the union of $\Lambda_{0}$ and invariant tori. Because of the compactness of $\overline{\Omega \backslash U}$, any orbit of some point $y \in \Sigma$ close to $x$ intersect $\partial U$ in two circles close to $\Gamma_{u}$ and $\Gamma_{s}$. The restriction of $F$ to $\Omega \backslash U$ is therefore a trivial fibration over $D$ whose fibers are finite length $S^{1}$-invariant affine cylinders, where the affine structure that defines the "axis" of each cylinder is given by the vector field $\mathcal{X}_{1}$ :

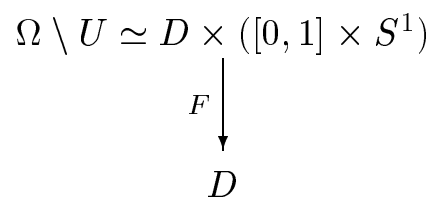

For instance, the circles $\Gamma_{u}$ and $\Gamma_{s}$ are represented in this trivialization by $\left(0,\left(0, S^{1}\right)\right)$ and $\left(0,\left(1, S^{1}\right)\right)$ respectively (see figure 3 ). For any $y \in \Omega \backslash U$ corresponding to the point $(c,(t, \tau))$ in the above trivialization, we denote by $\left[y_{u}, y_{s}\right]$ the $\mathcal{X}_{1}$-path corresponding to the axis $(c,([0,1], \tau))$. As we can assume that $U$ lies inside an open neighborhood of $m$ where Eliasson's normal form applies, $y_{u}$ and $y_{s}$ have complex coordinates $\left(y_{u / s, 1}, y_{u / s, 2}\right)$ satisfying

$$
\overline{y_{u / s, 1}} y_{u / s, 2}=f(c) \text {. }
$$

Note that the matrix $M$ in the statement of the proposition is equal to $d f(m)$.

Remark 6.2. We don't actually need the full statement of Eliasson's normal form, because higher order terms in the normal form wouldn't be of any trouble for the local computation of the monodromy (see $[16]$ ).

If $c \neq 0$, using formula (12) for the flow, $y_{s}$ can be joined to $y_{u}$ by letting the flow of $q_{1}$ act during a time $t(y)=\ln \left(\left|y_{u, 1}\right| /\left|y_{s, 1}\right|\right)$ and that of $q_{2}$ during a time

$$
s(y)=\arg \left(y_{u, 1} / y_{s, 1}\right) .
$$




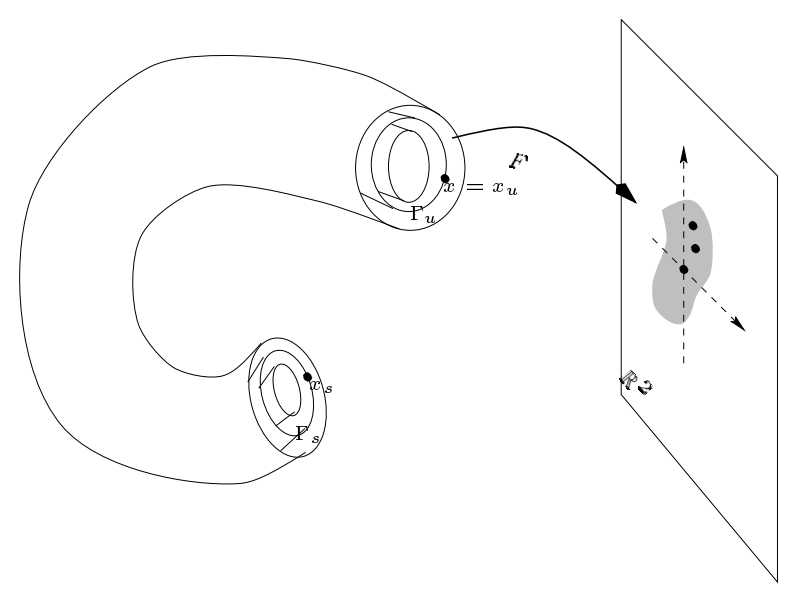

Figure 3: The fibration $F$ on $\overline{\Omega \backslash U}$

By this construction, we get a locally smooth family of loops on $\Lambda_{c}$, whose homology class at $c_{0}, \gamma_{1}\left(c_{0}\right)$, together with $\gamma_{2}\left(c_{0}\right)$, form the basis referred to in the statement of the proposition.

Let $\left[x_{u}, x_{s}\right]$ be the axis through $x$ (by definition, $x_{u}=x$ is on the local unstable manifold $P_{u}$ and $x_{s}$ on the local stable manifold $P_{s}$ ). If $y$ tends to $x$, then $y_{u}$ and $y_{s}$ tend to $x_{u}$ and $x_{s}$ respectively. Therefore, since $x_{u, 2}=x_{s, 1}=0, s(y) \sim \arg \left(x_{u, 1} \overline{x_{s, 2}}\right)+\arg (f(c))$ as $y$ tends towards $x$.

Now let $\mathcal{C}$ be a small loop around $x$ in $\Sigma$ such that its image by $F$ is a circle in $D$ through $c_{0}$, oriented in the trigonometric sense : letting $y$ run around $\mathcal{C}$, and with it, the loop $\gamma_{2}(c)$, amounts to increasing $\arg (c)$. Therefore, after such a revolution, $s(y)$ get increased or decreased by $2 \pi$, depending on the diffeomorphism $f$ being respectively orientation preserving or reversing. This means that the loop obtained is equal to the initial one composed by respectively plus or minus an $S^{1}$ orbit, which proves the proposition.

\subsection{Microlocal focus-focus}

We are now given a self-adjoint semi-classical completely integrable system $P_{1}(h), P_{2}(h)$ whose associated classical system presents a focus-focus singularity at a point $m$, as defined in the preceding section.

The aim of this section is the complete analysis of microlocal solutions around the critical point $m$ of the system :

$$
\left\{\begin{array}{l}
P_{1}^{E}(h) u_{h}^{E} \sim 0 \\
P_{2}^{E}(h) u_{h}^{E} \sim 0
\end{array}\right.
$$

in which $P_{j}^{E}$ is a regular deformation of $P_{j}$ with a parameter $E$ varying in a compact neighborhood of $0 \in \mathbb{R}^{2}$ (see definition 5.1).

The problem of globalizing these solution to a neighborhood of the whole critical leaf $\Lambda_{0}$ will be dealt with in the next section.

For now, the crucial point is the following : 
Proposition 6.4 The space of microlocal solution of (14) at $m$ has dimension $\leqslant 1$. In other words, if $u_{h}^{E},(h, E) \in \Gamma$ is a non-trivial solution of (14) at $m$, then for any other such solution $v_{h}^{E}$, there exists a constant $C^{E}(h) \in \mathbb{C}_{h}$, depending smoothly on $E$, such that :

$$
v_{h}^{E} \sim C(h)^{E} u_{h}^{E} \text { at } m .
$$

To prove this, we use a parameter dependent version of the normal form of [39] : let $Q_{j}(h)$ be the standard focus-focus operators on $\mathbb{R}^{2}$, that is, written in polar coordinates $(r, \theta)$ :

$$
Q_{1}=\frac{h}{i}\left(r \frac{\partial}{\partial r}+1\right) \quad \text { and } \quad Q_{2}=\frac{h}{i} \frac{\partial}{\partial \theta} .
$$

For notation convenience, we will write $\boldsymbol{P}(h)$ for the vector $\left(P_{1}(h), P_{2}(h)\right)$, and similarly for other quantities. Let $\boldsymbol{p}$ be the momentum map for the system $\left(p_{1}, p_{2}\right)$. The target space $\mathbb{R}^{2}$ of $\boldsymbol{p}$ will be identified with $\mathbb{C}$ by $\boldsymbol{p}=p_{1}+i p_{2}$. As we already saw, in suitable symplectic coordinates near $m$, there is a local diffeomorphism $F$ of $\mathbb{R}^{2}$ such that $\boldsymbol{p}=F(\boldsymbol{q})$. Thus,

$$
\boldsymbol{p}^{E}=f^{E} \circ F(\boldsymbol{q})
$$

The semi-classical normal form that we get is the following :

Lemma 6.5 ([39]) There exists a unitary Fourier integral operator $U^{E}(h)$, a microlocally invertible $2 \times 2$ matrix $\mathcal{M}^{E}(h)$ of pseudo-differential operators, and $\boldsymbol{\epsilon}^{E}(h) \in \mathbb{C}_{h}$, everything depending smoothly on $E$, such that, microlocally on a neighborhood of $m$ :

$$
\left(U^{E}\right)^{-1} \boldsymbol{P}^{E} U^{E}=\mathcal{M}^{E} \cdot\left(\boldsymbol{Q}-\boldsymbol{\epsilon}^{E}(h)\right)+O\left(h^{\infty}\right) .
$$

$\boldsymbol{\epsilon}^{E}$ admits a semi-classical asymptotic expansion of the form :

$$
\boldsymbol{\epsilon}^{E}(h) \sim \sum_{k \geqslant 0} h^{k} \boldsymbol{\epsilon}_{k}^{E}
$$

The first two terms satisfy:

$$
\begin{gathered}
\boldsymbol{\epsilon}_{0}^{E}=F^{-1} \circ\left(f^{E}\right)^{-1}(\overrightarrow{0}), \\
\boldsymbol{\epsilon}_{1}^{0}=-M^{-1} \cdot(\boldsymbol{r}(m)) .
\end{gathered}
$$

- $\boldsymbol{r}(m)$ is the value at $m$ of the sub-principal symbol of $\boldsymbol{P}$.

- $M$ is the value at 0 of principal symbol of $\mathcal{M}^{0}$. It is also defined by $M=d F(0)$, or, equivalently:

$$
d^{2} p^{0}(m)=M \cdot d^{2} q(0)
$$

Remark 6.3. We will show later (paragraph 6.7.4) that the expansion $\epsilon_{2}^{E}(h)=\mathfrak{I}\left(\boldsymbol{\epsilon}^{E}(h)\right)$ is, up to $O\left(h^{\infty}\right)$, independent on the choice of a Fourier integral operator $U(h)$. For the other "semiclassical invariant" $\epsilon_{1}^{E}(h)=\mathfrak{R}\left(\boldsymbol{\epsilon}^{E}(h)\right)$, this might not hold; however, will will prove thanks to corollary 6.10 that its formal infinite germ at $E=0$ is indeed invariant by a change of Fourier integral operator $U(h)$. 
Remark 6.4. If we apply this lemma to $E=h t$ for a bounded $n$-dimensional parameter $t$, we can apply remark 5.2 and express everything in terms of $t$. We obtain an asymptotic expansion $\tilde{\boldsymbol{\epsilon}}^{t}(h) \stackrel{\text { def }}{=} \boldsymbol{\epsilon}^{h t}(h)$ satisfying :

$$
\tilde{\boldsymbol{\epsilon}}_{0}^{t}=0, \quad \tilde{\boldsymbol{\epsilon}}_{1}^{t}=-M^{-1} \cdot\left(\boldsymbol{r}^{0}(m)+\frac{\partial f^{E}}{\partial E}\lceil E=0,(0) \cdot t) .\right.
$$

Thanks to this lemma, the solutions of (14) get transformed into those of simple differential operators on $\mathbb{R}^{2}$ for which we have explicit solutions. We can now turn to the proof of proposition 6.4. Assume that we know an exact solution of the system $u_{\text {exact }}^{E} \in \mathcal{S}^{\prime}\left(\mathbb{R}^{2}\right.$ ) (this will always occur as soon as the system admits a microlocal solution - see proposition 6.6). Let $u_{h}^{E}$ be any other microlocal solution near 0 .

Let $\Upsilon_{R}$ be the "vertical strip" $\{r<R\}$ in the cotangent bundle $T^{*} \mathbb{R}^{2}=((r, \theta),(\rho, \alpha))$. The Lagrangean leaves of the system in $\Upsilon_{R}$, deprived of a small ball $B$ around 0 , are non-singular, hence from proposition 5.1 there exists a constant $C^{E}(h)$ such that microlocally on $\Upsilon_{R} \backslash B$,

$$
u_{h}^{E} \sim C^{E}(h) u_{\text {exact }}^{E}
$$

(remember that such a formula involves uniformity with respect to $E$ ).

As in [11], we shall use this exact solution to construct a local $\left(\bmod O\left(h^{\infty}\right)\right)$ solution, microlocally equal to $u_{h}^{E}$ near the origin. Let us define $v_{h}^{E}$ by :

$$
\mathcal{F}_{h} v_{h}^{E}=\chi \mathcal{F}_{h} u_{h}^{E}+(1-\chi) \mathcal{F}_{h} C^{E} u_{\text {exact }}^{E},
$$

where $\chi$ is a compactly supported $C^{\infty}$ function with value 1 in a neighborhood of 0 . Then $v_{h}^{E}$ and $u_{h}^{E}$ are microlocally equal near the origin. Furthermore, $v_{h}^{E}$ is a microlocal solution of the system at each point of $\Upsilon_{R}$.

Now there exists differential operators $\hat{P}_{j}^{E}(h)$ such that for any $w \in \mathcal{S}^{\prime}\left(\mathbb{R}^{2}\right), \mathcal{F}_{h}\left(P_{j}^{E} w\right)=$ $\hat{P}_{j}^{E} \mathcal{F}_{h} w$. Differential operators preserve the support of distributions; therefore, since $\mathcal{F}_{h} v_{h}^{E}=$ $\mathcal{F}_{h} u_{\text {exact }}^{E}$ far from the origin, we have there $\hat{P}_{j}^{E} \mathcal{F}_{h} v_{h}^{E}=\mathcal{F}_{h}\left(P_{j}^{E} u_{\text {exact }}^{E}\right)=0$. So we have

$$
P_{j}^{E} v_{h}^{E} \sim 0
$$

near each point of $\Upsilon_{R}$, and

$$
\mathcal{F}_{h}\left(P_{j}^{E} v_{h}^{E}\right)=0
$$

far from 0 . We deduce from this that $P_{j}^{E} v_{h}^{E}$ satisfies the hypothesis of lemma 4.5 on $\Upsilon_{R}$, that is,

$$
P_{j}^{E} v_{h}^{E}=O\left(h^{\infty}\right) \text { on } \Upsilon_{R} \cap\{\rho=0\}
$$

We have thus at our disposal a solution $v_{h}^{E}$ of the system $P_{j}^{E} v_{h}^{E}=w_{j h}^{E}$ on $\mathbb{R}^{2}$ with $w_{j h}^{E}=$ $O\left(h^{\infty}\right)$ for $r<R$. On $\mathbb{R}^{2} \backslash\{0\}$ this is a regular linear differential system of the first order, whose solutions are of the form

$$
v_{h}^{E}=C s t e^{E}(h) \cdot u_{\text {exact }}^{E}+u_{\text {particular }}^{E} ;
$$


$u_{\text {exact }}^{E}$ is a solution of the homogeneous system, and $u_{\text {particular }}^{E}$ is a particular solution of the system that can be computed from $u_{\text {exact }}^{E}$ by the method of "variation of the constant". Explicitly, if we write

$$
v_{h}^{E}=g^{E} \cdot u_{\text {exact }}^{E},
$$

we find that the function $g^{E}$ must satisfy the following system, expressed in polar coordinates :

$$
\left\{\begin{array}{l}
\frac{\partial g^{E}}{\partial r}=\frac{i}{h} \frac{w_{1 h}^{E}}{r u_{\text {exact }}^{E}}=: a_{1 h}^{E} \\
\frac{\partial g^{E}}{\partial \theta}=\frac{i}{h} \frac{w_{2 h}^{E}}{u_{\text {exact }}^{E}}=: a_{2 h}^{E} .
\end{array}\right.
$$

If $\left(r_{0}, \theta_{0}\right)$ is any point with $0<r_{0}<R, g^{E}$ is integrated by the formula :

$$
g^{E}(r, \theta)=\int_{0}^{1} \boldsymbol{a}_{h}^{E}\left(r_{0}+t\left(r-r_{0}\right), \theta_{0}+t\left(\theta-\theta_{0}\right)\right) \cdot(r, \theta) d t+g^{E}\left(r_{0}, \theta_{0}\right) .
$$

It is easy to check from the explicit formula of $u_{\text {exact }}^{E}$ (see proposition 6.6) that $\boldsymbol{a}_{h}^{E}$ is uniformly $O\left(h^{\infty}\right)$ in the disc $r<R$; so with $\operatorname{Cste}^{E}(h)=g^{E}\left(r_{0}, \theta_{0}\right)$ we get in (15) that $u_{\text {particular }}^{E}$ is uniformly $O\left(h^{\infty}\right)$ near the origin.

The distribution $z_{h}^{E}=v_{h}^{E}-C s t e^{E} \cdot u_{\text {exact }}^{E}-u_{\text {particular }}^{E}$ is a solution of $P_{j}^{E} z_{h}^{E}=w_{j h}^{E}$ with support concentrated at the origin. Therefore, it is a finite sum of derivatives of the Dirac distribution. Now, the identity $P_{2}^{E} z_{h}^{E}=O\left(h^{\infty}\right)$ readily shows that the the coefficients involved in $z_{h}^{E}$ are uniformly $O\left(h^{\infty}\right)$, and so $z_{h}^{E}$ is.

Finally we are left with $v_{h}^{E}=C s t e^{E} \cdot u_{\text {exact }}^{E}+O\left(h^{\infty}\right)$ near 0 , which implies $v_{h}^{E} \sim C s t e^{E} \cdot u_{\text {exact }}^{E}$ near 0 . This, provided we prove proposition 6.6 , achieves the proof of proposition 6.4.

Remark 6.5. The proof shows that the constants $C^{E}(h)$ and $C s t e^{E}(h)$ are microlocally equal. Besides from the preceding arguments, this essentially comes from the local connectedness of the Lagrangean leaves $\Lambda_{0}^{E}$ for $E \neq 0$.

It remains now to check :

Proposition 6.6 We consider the system (14) on $\mathbb{R}^{2}$ with $\boldsymbol{P}^{E}(h)=\boldsymbol{Q}_{j}-\boldsymbol{\epsilon}^{E}(h)$, as previously defined. The following conditions are equivalent:

1. there exists a non-trivial microlocal solution $u_{h}^{E},(h, E) \in \Gamma$ of (14) on a neighborhood of the origin;

2. up to $O\left(h^{\infty}\right)$ modifications of the $P_{j}^{E}$, there exists an exact solution $u_{\text {exact }}^{E}$ of (14) in $\mathbb{R}^{2} \backslash\{0\}$ for $(h, E) \in \Gamma$;

3. $\frac{\epsilon_{2}^{E}(h)}{h} \in \mathbb{Z}+O\left(h^{\infty}\right)$ uniformly for $(h, E) \in \Gamma$.

Proof. Equivalence between 2. and 3. comes from the fact that at each point of $\mathbb{R}^{2} \backslash\{0\}$ the solutions of the system are spanned by the function :

$$
u_{\text {exact }}^{E}=\frac{1}{r} e^{i \frac{\epsilon_{1}^{E}(h)}{h} \ln r} e^{i \frac{\epsilon_{2}^{E}(h)}{h} \theta} .
$$


Therefore, there exists a solution around the origin if and only if $\frac{\epsilon_{2}^{E}}{h} \in \mathbb{Z}$.

Since $u_{\text {exact }}^{E}$ as defined above is an admissible functional in $\mathbb{R}^{2}$ depending smoothly on $E$, and a solution of the system on $\mathbb{R}^{2}$, it is also a microlocal solution. Hence 2 . implies 1 .

Now let $u_{h}^{E}$ be a non-trivial microlocal solution on a neighborhood $\Omega$ of 0 . From the uniqueness of microlocal solutions at points different from the origin (proposition 5.1), we deduce that on every simply connected open subset $\Omega_{\alpha}$ of $\Omega \backslash\{0\}$, there is a constant $\left(C^{E}\right)^{\alpha}(h)$ such that

$$
u_{h}^{E} \sim\left(C^{E}\right)^{\alpha} u_{\text {exact }}^{E}
$$

Then, going around 0 , the $\left(C^{E}\right)^{\alpha}$ 's must microlocally coincide. Hence the $e^{\epsilon_{2}^{\frac{E}{h}} \theta}$ must all microlocally coincide, uniformly for $(h, E) \in \Gamma$, which implies 3 .

Put together, the two propositions give the following result ("first quantization condition") :

Theorem 6.7 The system (14) has a non-trivial microlocal solution $u_{h}^{E}$ (with $(h, E) \in \Gamma$ ) on a neighborhood of $m$ if and only if

$$
\frac{\epsilon_{2}^{E}(h)}{h} \in \mathbb{Z}+O\left(h^{\infty}\right), \quad(h, E) \in \Gamma .
$$

Any other solution is a multiple of it.

Remark 6.6. As we already mentioned, microlocal solutions should be viewed as half-densities on the base space. Here, we can rewrite the half-density $u_{\text {exact }}^{E}|d x \wedge d y|^{\frac{1}{2}}$ as

$$
e^{i \frac{\epsilon_{1}^{E}}{h} \ln |r|} e^{i \frac{\epsilon_{2}^{E}}{h} \theta}\left|\frac{d x \wedge d y}{r^{2}}\right|^{\frac{1}{2}},
$$

and for $r \neq 0$, its modulus $\left|\frac{d x \wedge d y}{r^{2}}\right|^{\frac{1}{2}}$ is exactly the pull down of the canonical invariant Liouville half-density $\rho(\lambda)$ on $\Lambda_{0}^{E}$. In this sense, $u_{\text {exact }}^{E}$ is a normalized classical oscillatory half-density on every open subset $\Lambda^{E}$ of $\Lambda_{0}^{E}$ that projects diffeomorphically onto $X$ (thus excluding, for $E=0$, the vertical $(\xi, \eta)$-space in $\left.T^{*} X\right)$. Concerning the phase function $\varphi_{E}=\epsilon_{1,0}^{E} \ln |r|+\epsilon_{2,0}^{E} \theta$, a handy tool for its expression is the use of complex coordinates $z_{1}=x+i y$ and $z_{2}=\xi+i \eta$; we also write $\boldsymbol{\epsilon}_{0}^{E}=\epsilon_{1,0}^{E}+i \epsilon_{2,0}^{E}$, which leads to

$$
\varphi^{E}=\mathfrak{R}\left(\epsilon_{0}^{E} \ln \overline{z_{1}}\right)
$$

(Note that $\boldsymbol{\epsilon}_{0}^{E} \ln \overline{z_{1}}$ is not well-defined, but thanks to the assumption on $\epsilon_{2}^{E}(h)$, its real part is indeed well-defined modulo $2 \pi h \mathbb{Z}$.) We readily deduce that the graph of $\varphi^{E}$ in $T^{*} X$ is the set of $\left(z_{1}, z_{2}\right)$ such that $z_{2}=2 \frac{\partial \varphi^{E}}{\partial z_{1}}=\epsilon_{0}^{E} / \overline{z_{1}}$. Thus $\Lambda_{0}^{E}=\left\{\left(z_{1}, z_{2}\right) \in T^{*} X, \quad \overline{z_{1}} z_{2}=\boldsymbol{\epsilon}_{0}^{E}\right\}$. Note that this also holds if $E=0$, for then $\boldsymbol{\epsilon}_{0}^{E}=0$ and $\Lambda_{0}^{0}$ consists of the union of both coordinate planes $z_{1}=0$ and $z_{2}=0$. 


\subsection{Regularization of $[\kappa]$}

Before stating our principal result in the next section (theorem 6.9), we need to describe one more geometrical ingredient, related to the divergence of the integral of the sub-principal form $\kappa$ along paths through $m$.

We still let $\left(P_{1}(h), P_{2}(h)\right)$ be a semi-classical completely integrable system with a singular principal Lagrangean carrying a unique singular point $m$ of focus-focus type. Here again, we identify the target space of the momentum map $p$ with the complex plane $\mathbb{C}$. Thus $p=p_{1}+i p_{2}$. For small $c \in \mathbb{C}$, we denote by $\Lambda_{c}$ the Lagrangean leaf $\Lambda_{c}=\left\{p^{-1}(c)\right\}$, and $\kappa_{c}$ is as usual the sub-principal form on $\Lambda_{c}$.

Let $\gamma_{c}$ be a smooth family of loops on $\Lambda_{c}$ such that $\gamma_{0}$ is a simple loop through $m$ starting on the local unstable manifold of $\left(\Lambda_{0}, m\right)$, and coming back to $m$ via the local stable manifold. We know that the non-triviality of the monodromy implies that the map $c \rightarrow \gamma_{c}$ cannot be univalued, but proposition 6.3 gives a precise control over its multivaluedness. The purpose of this paragraph is to control the integral of the sub-principal form along such a family of paths, and in particular to determine its divergence principal part as $c \rightarrow 0$.

Let $M$ be the $2 \times 2$ real matrix such that $\mathcal{H}(p)=M . \mathcal{H}(q)$, where $q=q_{1}+i q_{2}$ (for the linearized flow, we always use the notations of 6.2 ). In accordance with lemma 6.5 we let

$$
\boldsymbol{\epsilon}_{1}=-M^{-1} \cdot r(m)
$$

where $r=r_{1}+i r_{2}$ is the joint sub-principal symbol of the system.

Proposition 6.8 For any multivalued smooth family $\gamma_{c}$ of loops on $\Lambda_{c}$ such that $\gamma_{0}$ is a simple loop through $m$ starting on the local unstable manifold of $\left(\Lambda_{0}, m\right)$, and coming back to $m$ via the local stable manifold, then

- the limit :

$$
I_{\gamma_{0}}(\kappa)=\lim _{\substack{c \rightarrow 0 \\ \text { along a ray }}}\left(\int_{\gamma_{c}} \kappa_{c}+\mathfrak{R}\left(\boldsymbol{\epsilon}_{1} \ln \left(M^{-1} c\right)\right)\right)
$$

exists,

- and its class modulo $2 \pi \mathfrak{I}\left(\boldsymbol{\epsilon}_{1}\right) \mathbb{Z}$ actually does not depend on $\gamma_{c}$, provided $\gamma_{0}$ satisfies the hypothesis;

- finally, this class is also given by the formula:

$$
I_{\gamma_{0}}(\kappa)=\lim _{(s, t) \rightarrow(0,0)}\left(\int_{A_{0}=\gamma_{0}(s)}^{B_{0}=\gamma_{0}(1-t)} \kappa+\mathfrak{R}\left(\boldsymbol{\epsilon}_{1}\right) \ln \left(r_{A_{0}} \rho_{B_{0}}\right)+\Im\left(\boldsymbol{\epsilon}_{1}\right)\left(\theta_{A_{0}}-\alpha_{B_{0}}\right)\right),
$$

where $(r, \theta, \rho, \alpha)$ are the polar symplectic coordinates near the origin defined in paragraph 6.2.

$I_{\gamma_{0}}(\kappa)$ will be called the principal value of $\int_{\gamma_{0}} \kappa$. 
Proof. Let $\left(\left[\gamma_{1}(c)\right],\left[\gamma_{2}(c)\right]\right)$ be the smooth basis of $\pi_{1}\left(\Lambda_{c}\right)$ defined in proposition 6.3. The homotopy class of $\gamma_{c}$ decomposes into

$$
\left[\gamma_{c}\right]=n_{1}(c)\left[\gamma_{1}(c)\right]+n_{2}(c)\left[\gamma_{2}(c)\right]
$$

for integers $n_{1}(c)$ and $n_{2}(c)$. On $\Lambda_{0}, \gamma_{2}(0)$ degenerates into a trivial loop, and by hypothesis we have

$$
\left[\gamma_{0}\right]=\left[\gamma_{1}(0)\right]
$$

But we know from proposition 6.3 that the group bundle $\pi_{1}\left(\Lambda_{c}\right) /\left\langle\gamma_{2}(c)\right\rangle \rightarrow c$ is trivial near $c=0$, with fibers isomorphic to $\mathbb{Z}$. Therefore, $n_{1}(c)$ must be identically equal to 1 for small $c$.

If $c$ is constrained into a sector $\Delta$ of the plane, then the family $\gamma_{c}$ must be continuous at zero, which implies that $n_{2}(c)$ is constant for small $c$. Since the integral of $\kappa_{c}$ along the $\mathcal{X}_{2}$-circle $\gamma_{2}(c)$ is equal to $-2 \pi r_{2}(m)+O(c)$, we have

$$
\lim _{\substack{c \rightarrow 0 \\ c \in \Delta}} \int_{n_{2}(c)\left[\gamma_{2}(c)\right]} \kappa_{c}=2 \pi \mathfrak{I}\left(\boldsymbol{\epsilon}_{1}\right) .
$$

This, provided we prove the first point of the proposition, shows the second point. It remains to show the convergence of $\int_{\gamma_{1}(c)} \kappa_{c}$, and the formula 17. We shall still assume that $c$ stays in a sector $\Delta$ and by $\gamma_{c}$ we denote a smooth representative of the first basis element $\left[\gamma_{1}(c)\right]$.

Let $f$ be the function on $\left(\mathbb{R}^{2}, 0\right)$ such that, in some neighborhood $\Omega$ of $m$ and some local symplectic coordinates around $m, p=f(q)$, so that $M=d f(0)$. If we let $\tilde{c}=f(c)$, the problem amounts to investigating the limit of

$$
\int_{\gamma_{\tilde{c}}} \kappa_{\tilde{c}}+\mathfrak{R}\left(\boldsymbol{\epsilon}_{1} \ln (c)\right),
$$

because $\ln \left(M^{-1} f(c)\right)=\ln (c)+O(c)$. Now, $\kappa_{\tilde{c}}$ is defined by

$$
\kappa_{\tilde{c}} \cdot \mathcal{X}_{p_{j}}=-r_{j}
$$

which also reads, in $\Omega$ :

$$
\left(\kappa_{\tilde{c}} \cdot \mathcal{X}_{q_{1}}, \kappa_{\tilde{c}} \cdot \mathcal{X}_{q_{2}}\right)=-(d f(q))^{-1} \cdot\left(r_{1}, r_{2}\right) .
$$

This means that $\kappa_{\tilde{c}}$ is, in $\Omega$, the sub-principal form on the $c$-level set of a system with principal symbol $q$ and sub-principal symbol $(d f(q))^{-1} . r$ (which we call $r$ again). So let us work with these data and forget the tilde.

As we shall see, we can even reduce the problem to a constant sub-principal symbol $r(0)(=$ $\left.-\boldsymbol{\epsilon}_{1}\right)$. This is due to the following "critical Poincaré lemma" (see [39]) : there exists a function $g$ on a neighborhood of $0 \in T^{*} \mathbb{R}^{2}$ and smooth functions $h_{j}$ on $\left(\mathbb{R}^{2}, 0\right)$ such that

$$
r_{j}=d g \cdot \mathcal{X}_{q_{j}}+h_{j}(q) .
$$

This implies that on $\Omega$, for non-zero $c$, the function

$$
K_{c} \equiv-g-h_{1}(c) \ln (r)-h_{2}(c) \theta \quad\left(\bmod 2 \pi h_{2}(c)\right)
$$


is a primitive of $\kappa_{c}$ on $\Lambda_{c}=\left\{r \rho e^{i(\alpha-\theta)}=c\right\}$. Suppose now that $A_{0}$ and $B_{0}$ are points on $\gamma_{0}$ lying respectively on the local unstable and stable manifolds of $\Lambda_{0}$. Then there exist smooth families of points $A_{c}$ and $B_{c}$ on $\Omega \cap \gamma_{c}([0,1])$ such that $\lim _{c \rightarrow 0}\left(A_{c}, B_{c}\right)=\left(A_{0}, B_{0}\right)$. We can write :

$$
\begin{gathered}
\int_{\gamma_{c}} \kappa_{c}=\int_{A_{c}}^{B_{c}} \kappa_{c}+K_{c}\left(A_{c}\right)-K_{c}\left(B_{c}\right) \\
=\int_{A_{c}}^{B_{c}} \kappa_{c}+g\left(B_{c}\right)-g\left(A_{c}\right)-h_{1}(c) \ln \left(r_{A_{c}} / r_{B_{c}}\right)-h_{2}(c)\left(\theta_{A_{c}}-\theta_{B_{c}}\right),
\end{gathered}
$$

which can also be written

$$
\begin{gathered}
\int_{A_{c}}^{B_{c}} \kappa_{c}+g\left(B_{c}\right)-g\left(A_{c}\right)-h_{1}(c) \ln \left(r_{A_{c}} \rho_{B_{c}}\right)-h_{2}(c)\left(\theta_{A_{c}}-\alpha_{B_{c}}\right) \\
+\left(h_{1}(c) \ln (|c|)-h_{2}(c) \arg (c)\right) .
\end{gathered}
$$

Everything but the last parenthesis has a smooth behavior as $c \rightarrow 0$ in $\Delta$, and that last parenthesis is nothing else than $\mathfrak{R}\left(\left(h_{1}(c)+i h_{2}(c)\right) \ln c\right)$. So there is a smooth function $I\left(A_{c}, B_{c}\right)$ such that :

$$
I\left(A_{c}, B_{c}\right)=\int_{\gamma_{c}} \kappa_{c}-\mathfrak{R}\left(\left(h_{1}(c)+i h_{2}(c)\right) \ln c\right) .
$$

Remark 6.7. Because of the monodromy matrix $\left(\begin{array}{ll}1 & 0 \\ 1 & 1\end{array}\right)$ (proposition 6.3), as $c$ loops in the positive sense around the origin, $\int_{\gamma_{c}} \kappa_{c}$ gets increased by

$$
\int_{\gamma_{2}(c)} \kappa_{c}=-2 \pi h_{2}(c)
$$

whereas $\mathfrak{R}\left(\left(h_{1}(c)+i h_{2}(c)\right) \ln c\right)=h_{1}(c) \ln (|c|)-h_{2}(c) \arg (c)$ gets increased by the same quantity. This shows that $I\left(A_{c}, B_{c}\right)$ is actually a univalued smooth function around $c=0$.

Because $\left(h_{1}(c)+i h_{2}(c)\right)=-\boldsymbol{\epsilon}_{1}+O(c)$, we deduce that if we fix a determination of $\ln c$ and make $c$ tend to 0 in $\Delta$, then

$$
\mathfrak{R}\left(\left(h_{1}(c)+i h_{2}(c)\right) \ln c+\boldsymbol{\epsilon}_{1} \ln c\right) \rightarrow 0
$$

Therefore, $I_{\gamma_{0}}(\kappa)$ exists and is equal to $I\left(A_{0}, B_{0}\right)$. Note that it is independent of $A_{0}$ and $B_{0}$, and hence equal to the limit of $I\left(A_{0}, B_{0}\right)$ as $A_{0}$ and $B_{0}$ tend to the critical point $m$. That means that the function $g$, being smooth at $m$, plays no role, justifying our assertion that the sub-principal symbols can be taken to be constant near $m$. This remark also yields the useful expression of $I_{\gamma_{0}}(\kappa)$ involving only objects living on $\Lambda_{0}$ given by formula $(17)$. 


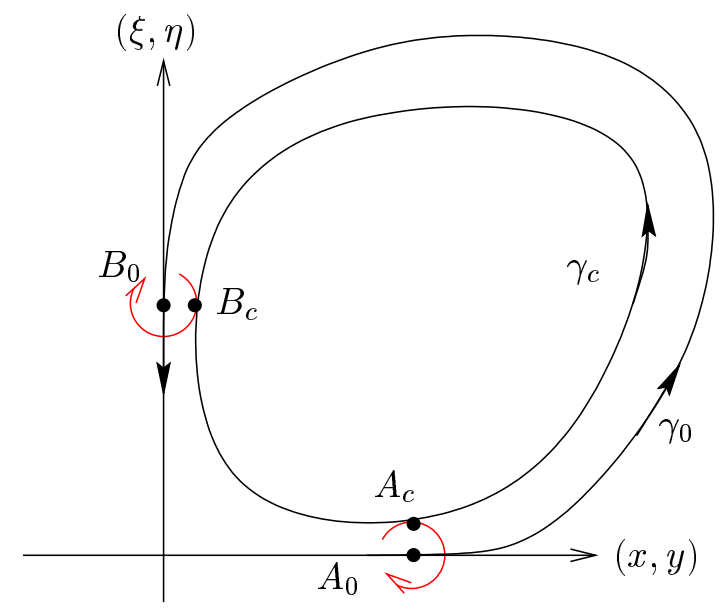

Figure 4: regularization of $\kappa$

\subsection{Global solutions}

Here is finally the main result of our work, namely the condition under which there exists a microlocal solution of the system (14) on a neighborhood of the whole critical Lagrangean $\Lambda_{0}$. The hypothesis are the same as in paragraph 6.5. From now on, $n$ is a generic integer, and not the dimension of the ambient symplectic manifold, which is 4 .

Theorem 6.9 Let $\epsilon_{j}^{E}=\epsilon_{j}^{E}(h)$ be the constants defined by lemma 6.5.

- There exists a $\mathbb{T}$-valued constant $\left(\lambda^{E}\right)^{\text {out }}=\left(\lambda^{E}\right)^{\text {out }}(h)$ admitting an asymptotic expansion in $\geqslant-1$ powers of $h$ whose terms are smooth functions of $E$, such that the system (14) admits a microlocal solution $u_{h}^{E},(h, E) \in \Gamma$, on a full neighborhood of $\Lambda_{0}$ if and only if the following two conditions are satisfied:

1. "first quantization condition":

$$
\frac{\epsilon_{2}^{E}(h)}{h} \in \mathbb{Z}+O\left(h^{\infty}\right)
$$

2. "second quantization condition" :

$$
\left(\lambda^{E}\right)^{\text {out }}+n \frac{\pi}{2}-\frac{\epsilon_{1}^{E}}{h} \ln (2 h)-2 \arg \Gamma\left(\frac{i \epsilon_{1}^{E} / h+1+n}{2}\right) \in 2 \pi \mathbb{Z}+O\left(h^{\infty}\right),
$$

where $\frac{\epsilon_{2}^{E}(h)}{h} \sim n \in \mathbb{Z}$.

- Moreover, the first two terms in the asymptotic expansion of $\left(\lambda^{E}\right)^{\text {out }}(h)$ are the following :

- for non-zero $E$,

$$
\begin{aligned}
& \frac{1}{h}\left(\int_{\gamma_{1}^{E}} \alpha_{0}^{E}-\epsilon_{1,0}^{E}+\epsilon_{1,0}^{E} \ln |\mathbf{c}|-\epsilon_{2,0}^{E} \arg \mathbf{c}\right)+ \\
& \quad+\left(\int_{\gamma_{1}^{E}} \kappa^{E}+\epsilon_{1,1}^{E} \ln |\mathbf{c}|-\epsilon_{2,1}^{E} \arg \mathbf{c}+\mu\left(\gamma_{1}^{E}\right) \frac{\pi}{2}\right),
\end{aligned}
$$


where $\gamma_{1}^{E}$ is a smooth family of loops on $\Lambda_{0}^{E}$ such that $\gamma_{1}^{0}$ satisfies the hypothesis of proposition 6.8, $\mathbf{c}=\epsilon_{1,0}^{E}+i \epsilon_{2,0}^{E}$, and $\mu$ is the Maslov cocycle on $\Lambda_{0}^{E}$. For small $E, \mu\left(\gamma_{1}^{E}\right)$ is a constant integer.

- for $E=0$,

$$
\frac{1}{h} \int_{\gamma_{1}^{0}} \alpha_{0}+I_{\gamma_{1}^{0}}(\kappa)+\mu\left(\gamma_{1}^{0}\right) \frac{\pi}{2}
$$

where $\kappa=\kappa^{0}$ is the sub-principal form of the system $\left(P_{1}^{0}, P_{2}^{0}\right)$.

The last point of this theorem follows from the expression for non-zero $E$ (recall that $\Lambda^{E}$ is then smooth) via proposition 6.8 : indeed, if we let $\tilde{\mathbf{c}}=\left(f^{E}\right)^{-1}(0)$, we have $\Lambda_{\tilde{\mathbf{c}}}=\Lambda_{0}^{E}$, and the family of loops $\gamma_{\tilde{\mathbf{c}}}$ satisfies the hypothesis of proposition 6.8 . The 1 -form $\kappa^{E}$ is defined by :

$$
\left(\kappa^{E} \cdot \mathcal{X}_{p_{1}^{0}}, \kappa^{E} \cdot \mathcal{X}_{p_{2}^{0}}\right)=\left(d f^{E}\right)^{-1} \cdot\left(r_{1}^{E}, r_{2}^{E}\right) .
$$

Writing $E$ as a function of $\tilde{\mathbf{c}}$, this shows that $\kappa^{E}$ is the sub-principal form of a system with principal symbol $\left(p_{1}^{0}, p_{2}^{0}\right)$ and sub-principal symbol $\left(d f^{E}\right)^{-1} \cdot\left(r_{1}^{E}, r_{2}^{E}\right)$. The value at $m$ of this sub-principal symbol is $r^{0}(m)$, hence proposition 6.8 implies that

$$
\int_{\gamma_{1}^{E}} \kappa^{E}+\mathfrak{R}\left(\boldsymbol{\epsilon}_{1}^{0} \ln M^{-1} \tilde{\mathbf{c}}\right)
$$

has a limit $I$ as $E \rightarrow 0$. Because $\boldsymbol{\epsilon}_{1}^{E}$ tends to $\boldsymbol{\epsilon}_{1}^{0}$ and $\mathbf{c}=\boldsymbol{\epsilon}_{0}^{E}$ is tangent to $M^{-1} \tilde{\mathbf{c}}$ at $\tilde{\mathbf{c}}=0$, this limit is equal to that of

$$
\int_{\gamma_{1}^{E}} \kappa^{E}+\mathfrak{R}\left(\boldsymbol{\epsilon}_{1}^{E} \ln \mathbf{c}\right)
$$

Moreover, we saw in (17) that it only depends on the value of the sub-principal form on $\Lambda_{0}$. But, on $\Lambda_{0},\left(d f^{0}\right)^{-1} \cdot\left(r_{1}^{0}, r_{2}^{0}\right)=r^{0}$. Therefore, $I$ is equal to the principal value $I_{\gamma_{0}}(\kappa)$ of a system with principal symbol $p^{0}$ and sub-principal symbol $r^{0}$.

The rest of the proof of this theorem will be split into two propositions 6.11 and 6.14 .

We know from the geometry of the Lagrangean fibration (section 6.4) that a neighborhood of the principal Lagrangean $\Lambda_{0}$ also contains regular tori $\Lambda_{0}^{E}, E \neq 0$ ( $E$ small) for which we already derived the quantization conditions (theorem 5.8). Therefore, one of our further tasks will be to show how these regular quantization conditions are contained in the "singular" quantization conditions announced above. This link is provided in paragraph 6.7.4

Finally, this theorem can be applied to "zoom" to a $O(h)$ scale : let, as in remark 5.2, $\left(\tilde{P}_{1}^{t}(h), \tilde{P}_{2}^{t}(h)\right)$ be the semi-classical completely integrable system defined by

$$
\tilde{P}_{j}^{t}(h) \stackrel{\text { def }}{=} P_{j}^{h t}(h),
$$

where $t$ is a 2-dimensional bounded parameter. From the constant $\boldsymbol{\epsilon}^{E}(h)=\left(\epsilon_{1}^{E}(h), \epsilon_{2}^{E}(h)\right)$ given by lemma 6.5 we define $\tilde{\boldsymbol{\epsilon}}^{t}(h) \stackrel{\text { def }}{=} \frac{1}{h} \boldsymbol{\epsilon}^{h t}(h)$. This $\tilde{\boldsymbol{\epsilon}}^{t}$ admits an asymptotic expansion in positive powers of $h$ whose coefficients are smooth functions of $t$. The principal term is given by :

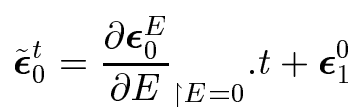




$$
=-M^{-1} \cdot\left({\frac{\partial f^{E}}{\partial E}}_{\lceil E=0}(0) \cdot t+r(m)\right)
$$

that is to say, in view of $(11)$,

$$
\tilde{\boldsymbol{\epsilon}}_{0}^{t}=-M^{-1} \cdot \tilde{r}^{t}(m)
$$

Here $\tilde{r}^{t}$ denotes the joint sub-principal symbol of the system $\left(\tilde{P}_{1}^{t}, \tilde{P}_{2}^{t}\right)$. Recall that its principal symbol is $\tilde{p}=p^{0}$ and does not depend on $t$.

Similarly, the holonomy coefficient $\left(\lambda^{E}\right)^{\text {out }}(h) \sim \sum_{k=-1}^{\infty}\left(\lambda^{E}\right)_{k}^{\text {out }}$ has a $t$-dependent version $\left(\tilde{\lambda}^{t}\right)^{\text {out }}(h)$ whose first terms are :

$$
\frac{1}{h}\left(\lambda^{0}\right)_{-1}^{\text {out }}+\left({\frac{\partial\left(\lambda^{h t}\right)_{-1}^{\text {out }}}{\partial h}}_{\lceil h=0}+\left(\lambda^{0}\right)_{0}^{\text {out }}\right)+O(h) .
$$

We know from the theorem that $\frac{1}{h}\left(\lambda^{0}\right)_{-1}^{\text {out }}=\frac{1}{h} \int_{\gamma_{1}^{0}} \alpha_{0}$. For the other term, using the identification $\boldsymbol{\epsilon}^{E}=\epsilon_{1}^{E}+i \epsilon_{2}^{E}$, we have, for non-zero $E$ :

$$
\begin{gathered}
\frac{\partial\left(\lambda^{E}\right)_{-1}^{\text {out }}}{\partial E}=\frac{\partial}{\partial E} \int_{\gamma_{1}^{E}} \alpha_{0}^{E}-\frac{\partial}{\partial E} \mathfrak{R}\left(\boldsymbol{\epsilon}_{0}^{E}-\boldsymbol{\epsilon}_{0}^{E} \ln \boldsymbol{\epsilon}_{0}^{E}\right) \\
=\frac{\partial}{\partial E} \int_{\gamma_{1}^{E}} \alpha_{0}^{E}+\mathfrak{R}\left(\ln \left(\boldsymbol{\epsilon}_{0}^{E}\right) \frac{\partial \boldsymbol{\epsilon}_{0}^{E}}{\partial E}\right) .
\end{gathered}
$$

Therefore,

$$
\begin{gathered}
{\frac{\partial\left(\lambda^{h t}\right)_{-1}^{\text {out }}}{\partial h}}_{\lceil h=0}+\left(\lambda^{0}\right)_{0}^{\text {out }}= \\
=\lim _{h \rightarrow 0}\left(\frac{\partial}{\partial h} \int_{\gamma_{1}^{E}} \alpha_{0}^{E}\right)+\int_{\gamma_{1}^{E}} \kappa^{E}+\Re\left(\left(\epsilon_{1}^{E}+\frac{\partial \epsilon_{0}^{E}}{\partial E} \cdot t\right) \ln \epsilon_{0}^{E}\right)+\mu\left(\gamma_{1}^{E}\right) \frac{\pi}{2},
\end{gathered}
$$

with $E=h t$. Using lemma 3.2, one easily shows that, for fixed $h=h_{0} \neq 0, \frac{\partial}{\partial h}{ }_{\uparrow h=h_{0}} \int_{\gamma_{1}^{E}} \alpha_{0}^{E}$ is equal to $\int_{\gamma_{1}^{E}} \delta \kappa^{E}$, where the 1 -form $\delta \kappa^{E}$ is given by

$$
\delta \kappa^{E} \cdot \mathcal{X}_{p^{E}}=-\frac{\partial f^{E}}{\partial E} \cdot t
$$

Therefore, the sum $\delta \kappa^{E}+\kappa^{E}$ is the sub-principal form of a system with principal symbol $p^{E}$ and sub-principal symbol $\frac{\partial f^{E}}{\partial E} \cdot t+r^{E}$, whose value at $m$ is equal to $\tilde{r}^{t}(m)$. It follows that

$$
\lim _{h \rightarrow 0} \int_{\gamma_{1}^{E}}\left(\delta \kappa^{E}+\kappa^{E}\right)+\mathfrak{R}\left(\left(\boldsymbol{\epsilon}_{1}^{E}+\frac{\partial \boldsymbol{\epsilon}_{0}^{E}}{\partial E} \cdot t\right) \ln \boldsymbol{\epsilon}_{0}^{E}\right)=I_{\gamma_{1}^{0}}\left(\delta \kappa^{0}+\kappa\right) .
$$

But $\delta \kappa^{0}+\kappa$ is the 1 -form on $\Lambda_{0}^{0}$ that takes the value $\frac{\partial f^{E}}{\partial E}\left\lceil E=0 . t+r^{0}\right.$ on $\left(\mathcal{X}_{p_{1}^{0}}, \mathcal{X}_{p_{2}^{0}}\right)$ : it is exactly the sub-principal form of the system $\left(\tilde{P}_{1}^{t}, \tilde{P}_{2}^{t}\right)$. We have proved the following result : 
Corollary 6.10 The system $\left(\tilde{P}_{1}^{t}, \tilde{P}_{2}^{t}\right)$ defined above admits a microlocal solution $u_{h}^{t}$ on a neighborhood of the critical Lagrangean $\Lambda_{0}$ if and only if the following two conditions are satisfied:

1. "first quantization condition" :

$$
\tilde{\epsilon}_{2}^{t}(h) \in \mathbb{Z}+O\left(h^{\infty}\right)
$$

2. "second quantization condition" :

$$
\left(\tilde{\lambda}^{t}\right)^{\text {out }}+n \frac{\pi}{2}-\tilde{\epsilon}_{1}^{t} \ln (2 h)-2 \arg \Gamma\left(\frac{i \tilde{\epsilon}_{1}^{t}+1+n}{2}\right) \in 2 \pi \mathbb{Z}+O\left(h^{\infty}\right),
$$

where $\tilde{\epsilon}_{2}^{t}(h) \sim n \in \mathbb{Z}$.

- Moreover, the first two terms in the asymptotic expansion of $\left(\tilde{\lambda}^{t}\right)^{\text {out }}(h)$ are the following :

$$
\frac{1}{h} \int_{\gamma_{1}^{0}} \alpha_{0}+I_{\gamma_{1}^{0}}\left(\tilde{\kappa}^{t}\right)+\mu\left(\gamma_{1}^{0}\right) \frac{\pi}{2}
$$

where $\gamma_{1}^{0}$ is a loop on $\Lambda_{0}$ that satisfies the hypothesis of proposition 6.8 and $\tilde{\kappa}$ is the sub-principal form of the system.

Remark 6.8. This corollary makes it easy to show that, provided the first quantization condition is satisfied, then the "semi-classical invariant" $\tilde{\epsilon}_{1}^{t}(h)$ is intrinsically defined by the microlocal behavior of the system around the critical point. This gives an a posteriori justification of the claim following lemma 6.5.

Indeed, let $n$ be the integer such that $\tilde{\epsilon}_{2}^{t}(h) \sim n$ (we shall prove in paragraph 6.7.4 that $\tilde{\epsilon}_{2}^{t}(h)$ - and even $\epsilon_{2}^{E}(h)$ - are intrinsically defined by the system around $m$, hence so is $n$ ).

For bounded $t, \tilde{\epsilon}_{1}^{t}(h)$ is uniformly bounded as $h \rightarrow 0$. As will be shown in the proof of proposition 6.11 , for any integer $n \in \mathbb{Z}$, the function $\frac{\Gamma\left(\frac{1}{2}(i x+1+n)\right)}{\Gamma\left(\frac{1}{2}(-i x+1+n)\right)}$ is analytic for $x \in \mathbb{R}$, which implies that the function $2 \arg \Gamma\left(\frac{1}{2}(i x+1+n)\right)$ is locally analytic. Therefore, plugging in $x=\tilde{\epsilon}_{1}^{t}(h)$ we obtain a semi-classical expansion in non-negative powers of $h$, whose terms are smooth $\mathbb{T}$-valued functions of $t$.

This in turn implies that the value of the cocycle $\left[\tilde{\lambda}^{t}(h)\right]$ on $\gamma_{1}^{0}$, which gives the "second quantization condition" of corollary 6.10 , can be written under the following form :

$$
\left[\tilde{\lambda}^{t}(h)\right]\left(\gamma_{1}^{0}\right) \equiv C^{t}(h)-\tilde{\epsilon}_{1}^{t}(h) \ln h+O\left(h^{\infty}\right),
$$

where $C^{t}(h)$ is a semi-classical expansion in $\geqslant-1$ powers of $h$ whose terms are smooth functions of $t$. Therefore, $-\tilde{\epsilon}_{1}^{t}(h)$ is uniquely defined as the coefficient of $\ln h$ in the expansion of $\left[\tilde{\lambda}^{t}(h)\right]\left(\gamma_{1}^{0}\right)$.

Now, if the system is changed outside a neighborhood of the critical point, then only $\left(\tilde{\lambda}^{t}\right)^{\text {out }}$ is perturbed, which means only a semi-classical perturbation that has an asymptotic expansion in powers of $h$, which does not affect the $\ln h$ coefficient.

Remark 6.9. In dimension 1, the singular Bohr-Sommerfeld conditions of Colin de Verdière and Parisse has been recently extended [13] to handle the case of multiple critical points of saddle 
type on the critical Lagrangean. In our case as well, if we restrict to focus-focus singularities, I believe that the results presented here can be extended to treat $k>1$ focus-focus points on $\Lambda_{0}$. This basically holds because the topology of such a $\Lambda_{0}$ is just a $k$-times pinched torus, and the monodromy if equal to the $k$-th power of the simple monodromy. However, including other kinds of singularities should require a more difficult analysis, starting from the topological description of $\Lambda_{0}$. Understanding the work of Fomenko (e.g. [25]), should probably help coping with this difficulty.

\subsubsection{The microlocal bundle}

As a starting point, we shall assume that the system fulfills the first quantization condition (theorem 6.7). This is of course a necessary condition for the existence of a global solution.

From this assumption, there exists a microlocal solution $\left(u_{h}^{E}\right)^{0}$ on a neighborhood of the critical point $m$. As in paragraph 5.2, one can thus form a microlocal line bundle $\mathcal{L}_{h}\left(\Lambda_{0}^{E}\right)$ over the topological manifold $\Lambda_{0}^{E}$, with well-defined holonomy, by means of the trivializations $(7)$. Of course this yields a family of line bundles depending on the 2-dimensional parameter $E$, but gathering the results of paragraph 5.5 and proposition 6.4 we see that the trivialization functions in (7) can be chosen to depend smoothly on $E$.

As a consequence, the holonomy of $\mathcal{L}_{h}\left(\Lambda_{0}^{E}\right)$ depends smoothly on $E$, in the sense that the elements $\left(c^{E}\right)^{\alpha \beta}$ of the Čech cocycle $c^{E}(h)$ representing it are $C^{\infty}$ functions of $E$ with values in $\overline{\mathbb{C}}_{h}{ }^{*}$.

From this picture, we already know the abstract form of the quantization condition we are looking for : it is exactly the parameter dependent version of proposition 5.5. In other words, there exists a microlocal solution $u_{h}^{E}$ on a neighborhood of $\Lambda_{0}$ if and only if for any family $\gamma^{E}$ of loops in $\Lambda_{0}^{E}$ that lie in that neighborhood, the value of $c^{E}(h)$ on $\gamma^{E}$ is uniformly $1+O\left(h^{\infty}\right)$ (here, we are viewing $c^{E}$ as a homomorphism from $\pi_{1}\left(\Lambda_{0}^{E}\right)$ to $\overline{\mathbb{C}}_{h}{ }^{*}$ ). Let us now explicit this condition.

Let $\Omega$ be a neighborhood of $m$ in $T^{*} X$ in which the normal form of lemma 6.5 applies. Then, as the system admits a microlocal solution on $\Omega$, any loop contained in $\Omega \cap \Lambda_{0}^{E}$ has microlocally trivial holonomy. But for every small $E \neq 0, \Omega$ contains a generator $\gamma_{2}^{E}$ of $\pi_{1}\left(\Lambda_{0}^{E}\right)$, namely the one associated with the $S^{1}$ rotational symmetry of the fibration (it is an integral curve of the vector field $\mathcal{X}_{2}$ of paragraph 6.3). Such a $\gamma_{2}^{E}$ also exists for $E=0$ but it is then homotopic to $\{m\}$. Anyhow, this yields :

$$
c^{E}\left(\gamma_{2}^{E}\right)=1+O\left(h^{\infty}\right)
$$

uniformly for small $E$ (this is of course due to the fulfillment of the first quantization condition).

Thus $c^{E}$ restricts to the quotient group $\pi_{1}\left(\Lambda_{0}^{E}\right) /\left(\gamma_{2}^{E}\right)$. For fixed $E$, every $\pi_{1}\left(\Lambda_{0}^{E}\right) /\left(\gamma_{2}^{E}\right)$ is isomorphic to $\mathbb{Z}$. What's more, proposition 6.3 implies that the group bundle $\pi_{1}\left(\Lambda_{0}^{E}\right) /\left(\gamma_{2}^{E}\right) \rightarrow$ $U \ni E$ over a neighborhood $U$ of 0 in $\mathbb{R}^{2}$ is trivial :

$$
\pi_{1}\left(\Lambda_{0}^{E}\right) /\left(\gamma_{2}^{E}\right) \simeq U \times \mathbb{Z}
$$

Let $\bar{\gamma}_{1}^{E}$ be the generator of $\pi_{1}\left(\Lambda_{0}^{E}\right) /\left(\gamma_{2}^{E}\right)$ equal to $(E, 1)$ in the above trivialization. The "second quantization condition" is now the requirement that $c^{E}\left(\bar{\gamma}_{1}^{E}\right)=1+O\left(h^{\infty}\right)$. 
Let us now examine that statement more closely. Recall that the line bundle $\mathcal{L}_{h}\left(\Lambda_{0}^{E}\right)$ was defined through a set of local trivializations subordinated to a cover $\cup \Omega_{\alpha}$ of a neighborhood of $\Lambda_{0}$ in $T^{*} X$, such that on every $\Omega_{\alpha}$ existed a non-trivial microlocal solution $\left(u_{h}^{E}\right)^{\alpha}$ of the system. We can assume that the critical point $m$ is contained in a unique element of that cover, which we will denote by $\Omega_{0}$. Let us pick up a simple loop $\gamma_{1}^{0}(t), t \in[0,1]$, in $\Lambda_{0}$ representing the quotient class $\bar{\gamma}_{1}^{0}$, and enumerate $\Omega_{0}, \Omega_{1}, \ldots, \Omega_{\ell}, \Omega_{0}$ the $\Omega_{\alpha}$ 's encountered by $\gamma_{1}^{0}(t)$ on its way from $m$ back to $m$ again. We will here choose $\gamma_{1}^{0}$ so that it goes away from $m$ via the local stable manifold of $\Lambda_{0}$ and returns back to $m$ via the local unstable manifold. Also, we will suppose that $\Omega_{0} \cup \Omega_{1} \cup \Omega_{\ell} \subset \Omega$.

Now for every fixed $E$, we choose a representative $\gamma_{1}^{E}$ of $\bar{\gamma}_{1}^{E}$. Because of the triviality of the fibration outside $m$ (13), the paths $\gamma_{1}^{E}$ can be homotopically deformed so as to be close to $\gamma_{1}^{0}$, in the sense that their images enter $\bigcup_{i=0}^{\ell} \Omega_{i}$.

Then by definition, the value of $c^{E}$ on $\gamma_{1}^{E}$ is the product :

$$
c^{E}\left(\gamma_{1}^{E}\right)=\left(c^{E}\right)^{0,1}\left(c^{E}\right)^{1,2} \cdots\left(c^{E}\right)^{\ell-1, \ell}\left(c^{E}\right)^{\ell, 0} .
$$

We are going to calculate this expression by splitting it in two parts : a local one - the product $\left(c^{E}\right)^{0,1}\left(c^{E}\right)^{\ell, 0}$ - and an outer one - the rest. For this purpose, we are free to fix the solutions $\left(u_{h}^{E}\right)^{i}$ on $\Omega_{i}$ used for the local trivialization of $\mathcal{L}_{h}\left(\Lambda_{0}^{E}\right)$; let us make the following choice :

- choice 1 : for $i \neq 0,1, \ell,\left(u_{h}^{E}\right)^{i}$ is any classical oscillatory integral constructed as in proposition 5.7, with invariant half density given by the canonical Liouville density on $\Lambda_{0}^{E}$.

For $i=0,1, \ell$, we make use of the local analysis of the preceding paragraph 6.5. To begin with, we transform the system on $\Omega$ to the normal form of lemma 6.5 by a unitary Fourier integral operator $U(h)$. The microlocal solutions of the transformed system on $\Omega$ are spanned by :

$$
u_{\text {exact }}^{E}=\frac{1}{r} e^{i \frac{\epsilon_{1}^{E}}{h} \ln r} e^{i \frac{\epsilon_{2}^{E}}{h} \theta} .
$$

Because of the first quantization condition, we know $\frac{\epsilon_{2}^{E}}{h}$ to be microlocally equal to an integer $n \in \mathbb{Z}$. For any real $\varepsilon$, let $u_{\varepsilon, n}$ be the tempered distribution on $\mathbb{R}^{2}$ :

$$
u_{\varepsilon, n}=\frac{1}{r} e^{i n \theta} e^{i \varepsilon \ln r},
$$

so that $u_{\text {exact }}^{E}=u_{\frac{\epsilon_{1}^{E}}{h}, \frac{\epsilon_{2}^{E}}{h}}$. The distribution $u_{\varepsilon, n}$ is a solution of the system

$$
\left\{\begin{array}{l}
\frac{1}{i} \frac{\partial}{\partial \theta} u_{\varepsilon, n}=n u_{\varepsilon, n} \\
\frac{1}{i}\left(r \frac{\partial}{\partial r}+1\right) u_{\varepsilon, n}=\varepsilon u_{\varepsilon, n}
\end{array}\right.
$$

on $\mathbb{R}^{2}$, and it is easy to see that its Fourier transform is a solution of the same system with $\varepsilon$ changed to $-\varepsilon$. Therefore $u_{\frac{\epsilon_{1}^{E}}{h}, \frac{\epsilon_{2}^{E}}{h}}$ and $\mathcal{F}_{h}^{-1}\left(u_{\frac{-\epsilon_{1}^{E}}{h}, \frac{\epsilon_{2}^{E}}{h}}\right)$ are two solutions of our transformed system on $\Omega$. This allows us to complete our choice of local solutions by the following :

- choice 2: $\begin{cases}\left(u_{h}^{E}\right)^{0} \sim U\left(u_{\frac{\epsilon_{1}^{E}}{h}, \frac{\epsilon_{2}^{E}}{h}}\right) & \text { on } \Omega_{0} \\ \left(u_{h}^{E}\right)^{1} \sim U\left(u_{\epsilon_{1}^{E}}, \frac{\epsilon_{2}^{E}}{h}\right) & \text { on } \Omega_{1} \\ \left(u_{h}^{E}\right)^{\ell} \sim U\left(\mathcal{F}_{h}^{-1} u_{\frac{-\epsilon_{1}^{E}}{h}, \frac{\epsilon_{2}^{E}}{h}}\right) & \text { on } \Omega_{\ell}\end{cases}$ 


\subsubsection{The local holonomy at $m$}

We give here an explicit expression for the product $\left(c^{E}\right)^{1,0}\left(c^{E}\right)^{0, \ell}$, which is defined to be the constant $C^{E}(h)$ such that, on $\Omega_{0} \cap \Omega_{\ell},\left(u_{h}^{E}\right)^{0} \sim C^{E}\left(u_{h}^{E}\right)^{\ell}$.

Proposition 6.11 The unique microlocal constant $C^{E}(h)$ such that $\left(u_{h}^{E}\right)^{0} \sim C^{E}\left(u_{h}^{E}\right)^{\ell}$ on $\Omega_{0} \cap \Omega_{\ell}$ is given by :

$$
C^{E}(h) \sim i^{-n}(2 h)^{i \epsilon_{1}^{E} / h} \frac{\Gamma\left(\frac{i \epsilon_{1}^{E} / h+1+n}{2}\right)}{\Gamma\left(\frac{-i \epsilon_{1}^{E} / h+1+n}{2}\right)},
$$

with $\frac{\epsilon_{2}^{E}}{h} \sim n \in \mathbb{Z}$

Proof. Applying the Fourier integral operator $U_{h}$, we see that $C_{h}^{E}$ is also defined to be the constant such that, microlocally on $\Omega_{0} \cap \Omega_{\ell}$,

$$
\mathcal{F}_{h} u_{\frac{\epsilon_{1}^{E}}{h}, \frac{\epsilon_{2}^{E}}{h}} \sim C_{h}^{E} u_{\frac{-\epsilon_{1}^{E}}{h}, \frac{\epsilon_{2}^{E}}{h}} .
$$

It turns out that the constant $C_{h}^{E}$ thus defined is a microlocal version of an exact problem concerning the homogeneous distributions $u_{\varepsilon, n}$ on $\mathbb{R}^{2}$.

We know that the tempered distributions $u_{\varepsilon, n}$ are solutions of the system (18) in $\mathbb{R}^{2}$. They are in fact the only ones - up to multiplicative constants, naturally. To see this, one first restricts to $\mathbb{R}^{2} \backslash\{0\}$, where the result is standard, and then prove that the system (18) cannot admit linear combinations of derivatives of the Dirac distribution as solutions. Now, $\mathcal{F}^{-1} u_{-\varepsilon, n}$ is also a tempered distribution and a solution of the same system, which leads to the first point of the following proposition :

Proposition $6.12([4, \mathbf{2 6}]) \quad$ 1. For any $(\varepsilon, n) \in \mathbb{R} \times \mathbb{Z}$, there exists a unique constant $C(\varepsilon, n)$ such that:

$$
\mathcal{F} u_{\varepsilon, n}=C(\varepsilon, n) u_{-\varepsilon, n}
$$

2. $C(\varepsilon, n)$ has the following expression :

$$
C(\varepsilon, n)=i^{-|n|} 2^{i \varepsilon} \frac{\Gamma\left(\frac{i \varepsilon+1+|n|}{2}\right)}{\Gamma\left(\frac{-i \varepsilon+1+|n|}{2}\right)} .
$$

The computation of $C(\varepsilon, n)$ is done by testing $u_{\varepsilon, n}$ on derivatives of Gaussian functions $\left(\frac{\partial}{\partial z}\right)^{n} e^{\frac{z \bar{z}}{2}}$ and $\left(\frac{\partial}{\partial \bar{z}}\right)^{n} e^{\frac{z \bar{z}}{2}}\left(z=r e^{i} \theta\right)$.

Remark 6.10. Apparently, this formula appeared for the first time in Tate's thesis, during the year 1950. However, it has not been published until 1967, when it appeared in the book [4, chapter XV]. But at the same time as Tate wrote his thesis, the idea was in the air. Gelfand was studying homogeneous distributions in the real and published a closely related formula in [27]. 
The complex version, that is, exactly the result mentioned in the above proposition, appeared in the addendum of the French edition of the book by Gelfand \& al. on distributions [26], where it is claimed to be published for the first time; the author was certainly unaware of Tate's work. Also interestingly enough, few years before (1951), a slightly different version of the same formula can be found in Bochner's work [2]. It is actually a generalization to homogeneous distributions on $\mathbb{R}^{k}$.

Now, let us (momentarily) denote by $D(\varepsilon, n)$ the quantity :

$$
D(\varepsilon, n)=i^{-n} 2^{i \varepsilon} \frac{\Gamma\left(\frac{i \varepsilon+1+n}{2}\right)}{\Gamma\left(\frac{-i \varepsilon+1+n}{2}\right)} .
$$

The Gamma functions involved are analytic functions of $\varepsilon$ that, for negative odd $n$, have simple poles at $\varepsilon=0$. The quotient $D(\varepsilon, n)$ is therefore analytic - with no pole - on the real line (we won't consider here analytic continuation to complex $\varepsilon$ ). More precisely, for $n=-2 m-1, m \geqslant 0$, as the residues of the numerator and the denominator are the same, we have : $D(0,-2 m-1)=$ $-i^{-n}$. For non-zero $\varepsilon$, we can use the complement relation to prove that $D(\varepsilon, n)=D(\varepsilon,-n)$. Then of course this also holds for $\varepsilon=0$. Since $D(\varepsilon, n)$ and $C(\varepsilon, n)$ coincide for $n \geqslant 0$, we deduce that they are equal for all $n \in \mathbb{Z}$.

Finally, using the relation $\mathcal{F}_{h} u(\xi, \eta)=\frac{1}{h} \mathcal{F} u\left(\frac{\xi}{h}, \frac{\eta}{h}\right)$, we terminate the proof of proposition 6.11 .

Remark 6.11. One of the important features of the constants $C(\varepsilon, n)$ (and hence of $C^{E}$ ) is that they have modulus one. It might be interesting to see that, without any explicit calculation, this comes as a consequence of the unitarity of the Fourier transform with respect to the $L^{2}$ norm. As this does not appear - to my knowledge - in the literature, here is the argument :

Acting on functions of the Schwartz space $\mathcal{S}\left(\mathbb{R}^{2}\right), \mathcal{F}$ commutes with the operator $\frac{1}{i} \frac{\partial}{\partial \theta}$, and hence leaves stable its eigenspaces $\mathcal{E}_{n}$, associated to the eigenvalues $2 \pi n$. These are of course the subspaces of the Fourier series decomposition in $\theta$ variable $: \mathcal{E}_{n}=\left\{e^{i n \theta} f(r)\right\}$. Here, $f$ is a rapidly decreasing function. Therefore, there exists a transformation $\mathcal{F}^{n}$ on $\mathcal{E}_{n}$ such that

$$
\mathcal{F}\left(e^{-i n \theta} f(r)\right)(\rho, \alpha)=e^{-i n \alpha} \mathcal{F}^{n}(f)(\rho) .
$$

$\mathcal{F}$ being an isometry for the norms $L^{2}\left(\mathbb{R}^{2}, r d r d \theta\right)$ and $L^{2}\left(\mathbb{R}^{2}, \rho d \rho d \alpha\right)$, we deduce that $\mathcal{F}^{n}$ is an isometry from $L^{2}\left(\left[0, \infty[, r d r)\right.\right.$ into $L^{2}([0, \infty[, \rho d \rho)$.

On the other hand, let $\mathcal{G}$ be the transformation assigning to a function $f(r) \in L^{2}([0, \infty[, r d r)$ the function

$$
\mathcal{G}(f)(\varepsilon)=\int_{0}^{\infty} r^{i \varepsilon-1} f(r) r d r
$$

Lemma 6.13 $\mathcal{G}$ is an isometry from $L^{2}\left(\left[0, \infty[, r d r)\right.\right.$ into $L^{2}(\mathbb{R}, d \varepsilon)$.

Proof. The change of variable $x=\ln r$ allows us to reduce the problem to the unitarity of the Fourier transform : let $g(\varepsilon)=\mathcal{G}(f)(\varepsilon)$. We find $g(\varepsilon)=\mathcal{F}\left(f\left(e^{x}\right) e^{x}\right)(-\varepsilon)$, which gives $\|g\|_{2}=\left\|f\left(e^{x}\right) e^{x}\right\|_{2}=\int_{0}^{\infty}|f(r)|^{2} r d r=\|f\|_{2}$. 
We know from the definition of $C(\varepsilon, k)$ that for any function of the form

$$
\varphi=\mathcal{F}\left(e^{-i n \theta} f(r)\right),
$$

we have : $\left\langle\mathcal{F}\left(u_{(\varepsilon, k)}\right), \varphi\right\rangle=C(\varepsilon, k)\left\langle u_{(-\varepsilon, k)}, \varphi\right\rangle$, that is :

$$
\left\langle e^{i k \theta} r^{i \varepsilon-1}, \mathcal{F} \varphi\right\rangle=C(\varepsilon, k)\left\langle e^{i k \alpha} \rho^{-i \varepsilon-1}, \varphi\right\rangle,
$$

or :

$$
\left\langle r^{i \varepsilon-1}, e^{i(k-n)(\theta+\pi)} f(r)\right\rangle=C(\varepsilon, k)\left\langle\rho^{-i \varepsilon-1}, e^{i(k-n) \alpha}\left(\mathcal{F}^{n} f\right)(\rho)\right\rangle .
$$

Both sides of the equality vanish if $n-k \neq 0$. So let us assume $k=n$. We obtain :

$$
\mathcal{G}(f)(\varepsilon)=(-1)^{n} C(\varepsilon, n) \mathcal{G} \mathcal{F}^{n} f(-\varepsilon),
$$

which is best depicted by the following commutative diagram :

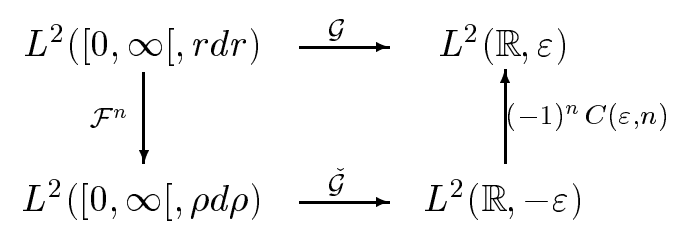

All transformation involved $\left(\mathcal{G}, \mathcal{F}^{n}\right.$ and $\varepsilon \rightarrow-\varepsilon$ ) being unitary ones, one can indeed deduce that $\varepsilon \mapsto C(\varepsilon, n)$ is a function whose modulus is 1 .

Remark 6.12. The above introduced transformations $\mathcal{G}$ and $\mathcal{F}^{n}$ are related to Mellin's and Hankel's transforms $\mathcal{M}$ and $\mathcal{H}_{n}$ respectively (see [14] for their definitions). More precisely, we have :

$$
(\mathcal{G} f)(\varepsilon)=(\mathcal{M} f)(s=i \varepsilon+1) \text { and } \mathcal{F}^{n}=i^{-n} \mathcal{H}_{n} .
$$

(see for instance [17]). The previous remark thus yields the following remarkable relation between these transformations :

$$
\forall f, \quad \mathcal{M} f(i \varepsilon+1)=i^{n} C(\varepsilon, n) \cdot\left(\mathcal{M H}_{n} f\right)(-i \varepsilon+1) .
$$

Remark 6.13. Formula (20) allows us to give another proof of the expression of $C(\varepsilon, n)$. First of all, since $\mathcal{H}_{-n}=(-1)^{n} \mathcal{H}_{n}$, it readily gives $C(\varepsilon, n)=C(\varepsilon,-n)$. Now for $n \geqslant 0$ choose $f_{n}=r^{n} e^{-\frac{r^{2}}{2}} . f_{n}$ is fixed by Hankel's transform of order $n$. On the other hand, it is easy to see that $\mathcal{M} f_{n}(s)=2^{\frac{s+n}{2}-1} \Gamma\left(\frac{s+n}{2}\right)$. This yields :

$$
C(\varepsilon, n)=i^{-n} \frac{2^{s / 2} \Gamma((s+n) / 2)}{2^{\bar{s} / 2} \Gamma((\bar{s}+n) / 2)}, \text { with } s=i \varepsilon+1, \bar{s}=-i \varepsilon+1,
$$

which is easily identified with formula (19). 


\subsubsection{The outer holonomy}

Let us turn now to the study of the "outer holonomy", that is, the product

$$
\left(C^{E}\right)^{\text {out }}=\left(c^{E}\right)^{1,2} \cdots\left(c^{E}\right)^{\ell-1, \ell} .
$$

Recall that its quotient by the local holonomy $C^{E}$ defined in the previous paragraph gives the value of the Čech cocycle $c^{E}$ on $\gamma_{1}^{E}$.

Proposition 6.14 $\bullet\left|\left(C^{E}\right)^{\text {out }}\right|=1+O\left(h^{\infty}\right)$;

- $\arg \left(\left(C^{E}\right)^{\text {out }}\right) \in \mathbb{T}$ admits an asymptotic expansion in $\geqslant-1$ powers of $h$, whose terms are smooth functions of $E$;

- for non zero $E$, the first two terms in that expansion are the following :

$$
\begin{aligned}
& \frac{1}{h}\left(\int_{\gamma_{1}^{E}} \alpha_{0}^{E}-\epsilon_{1,0}^{E}+\epsilon_{1,0}^{E} \ln |\mathbf{c}|-\epsilon_{2,0}^{E} \arg \mathbf{c}\right)+ \\
& \quad+\left(\int_{\gamma_{1}^{E}} \kappa^{E}+\epsilon_{1,1}^{E} \ln |\mathbf{c}|-\epsilon_{2,1}^{E} \arg \mathbf{c}+\mu\left(\gamma_{1}^{E}\right) \frac{\pi}{2}\right),
\end{aligned}
$$

where $\mathbf{c}=\epsilon_{1,0}^{E}+i \epsilon_{2,0}^{E}$.

Proof. For non zero $E, \Lambda_{0}^{E}$ is a regular Lagrangean torus, and we know from proposition 5.6 that $\left|c^{E}\left(\gamma_{1}^{E}\right)\right| \sim 1$. This, together with the fact that $\left|C^{E}\right| \sim 1$ too, implies that $\left|\left(C^{E}\right)^{\text {out }}\right| \sim 1$ as well. Then of course, since we already knew that $\left(C^{E}\right)^{\text {out }}$ is a smooth function of $E$, the results also holds if $E=0$.

The second point in the proposition is essentially due to our choice of the microlocal solution $\left(u_{h}^{E}\right)^{\ell}$ on $\Omega_{\ell}$. The distribution

$$
\frac{1}{2 \pi i h} \int e^{\frac{i}{h}\left(\langle(x, y),(\xi, \eta)\rangle-\epsilon_{1}^{E} \ln \rho+\epsilon_{2}^{E} \alpha\right)} \frac{|d \xi \wedge d \eta|}{\rho}
$$

(by $(\rho, \alpha)$ we always denote polar coordinates for $(\xi, \eta)$ in the fiber of $\left.T^{*} \mathbb{R}^{2}\right)$ is microlocally on $\Omega_{\ell}$ equal to a classical oscillatory integral on $\Lambda_{0}^{E} \cap \Omega_{\ell}$, associated with the canonical Liouville half density on $\Lambda_{0}^{E}$, and smoothly depending on $E$.

Remark 6.14. This is of course the pendant of remark 6.6. Here as well, the phase function $\varphi^{E}=\langle(x, y),(\xi, \eta)\rangle-\epsilon_{1,0}^{E} \ln \rho+\epsilon_{2,0}^{E} \alpha$ is best expressed in terms of complex coordinates $z_{1}$ and $z_{2}$ : we have

$$
\varphi^{E}\left(z_{1}, z_{2}\right)=\mathfrak{R}\left(\overline{z_{1}} z_{2}-\epsilon_{0}^{E} \ln z_{2}\right) .
$$

Now, since $\left(u_{h}^{E}\right)^{\ell}$ was defined to be the image of that distribution by an classical Fourier integral operator, it is also a classical Lagrangean distribution, as are all the other solutions $\left(u_{h}^{E}\right)^{i}, i \neq 0$. We deduce from proposition 5.6 and paragraph 5.5 that for each $i=1, \ldots, \ell-1$, 
the argument of $\left(c^{E}\right)^{i, i+1}$ admits a semi-classical expansion in $\geqslant-1$ powers of $h$ whose terms smoothly depend on $E$, as functions with values in $\mathbb{T}$. Hence so does their product $\left(C^{E}\right)^{\text {out }}$.

We turn now to the last point of the proposition, which requires a closer look at the principal phases of the solutions near the critical point $m$. Indeed, we know from the non-singular analysis of section 5 that the principal phases together with a section of the Keller-Maslov line bundle form an invariantly defined object that gives the first two terms of the Čech cocycle $\lambda^{E}(h)$.

First, let us extend the open sets $\Omega_{1}$ and $\Omega_{\ell}$ to be both equal to $\Omega \backslash\{m\}$. This is possible as the microlocal solutions $\left(u_{h}^{E}\right)^{1}$ and $\left(u_{h}^{E}\right)^{\ell}$ are actually well-defined on all $\Omega$. This ensures that for every non zero $E, \bigcup_{i=1}^{\ell} \Omega_{i}$ is a full cover of the image of $\gamma_{1}^{E}$. Still assuming that $E$ is non zero, we get from theorem 5.8 that $\arg \left(c^{E}\left(\gamma_{1}^{E}\right)\right)$ is, up to $O(h)$ (and modulo $2 \pi$ ), equal to

$$
\frac{1}{h} \int_{\gamma_{1}^{E}} \alpha_{0}^{E}+\int_{\gamma_{1}^{E}} \kappa^{E}+\mu\left(\gamma_{1}^{E}\right) \frac{\pi}{2}
$$

On the other hand, $c^{E}\left(\gamma_{1}^{E}\right)$ is by definition the value of the product

$$
\left(C^{E}\right)^{\text {out }}\left(c^{E}\right)^{\ell, 1}
$$

The problem is thus reduced to the computation of $\arg \left(c^{E}\right)^{\ell, 1}$. It is unchanged by conjugation by a Fourier integral operator on $\Omega$.

Let us denote by $\varphi_{1}^{E}\left(z_{1}\right)$ and $\varphi_{\ell}^{E}\left(z_{1}, z_{2}\right)$ the phase functions of the oscillatory functions $u_{\frac{\epsilon_{1}^{E}}{h}, \frac{\epsilon_{2}^{E}}{h}}$ and $\mathcal{F}_{h}^{-1} u_{\frac{-\epsilon_{1}^{E}}{h}, \frac{\epsilon_{2}^{E}}{h}}$. They have the following expressions (modulo $2 \pi h \mathbb{Z}$ ) :

$$
\varphi_{1}^{E}\left(z_{1}\right) \equiv \mathfrak{R}\left(\boldsymbol{\epsilon}_{0}^{E} \ln \overline{z_{1}}\right) \text { and } \varphi_{\ell}^{E}\left(z_{1}, z_{2}\right) \equiv \mathfrak{R}\left(\overline{z_{1}} z_{2}-\boldsymbol{\epsilon}_{0}^{E} \ln z_{2}\right) .
$$

As we already saw, for non zero $E$, they represent - of course - the same Lagrangean manifold $\Lambda_{0}^{E}=\left\{\overline{z_{1}} z_{2}=\boldsymbol{\epsilon}_{0}^{E}\right\}$ (the terms $\mathfrak{R}\left(\boldsymbol{\epsilon}_{0}^{E} \ln \overline{z_{1}}\right)$ and $\mathfrak{R}\left(\boldsymbol{\epsilon}_{0}^{E} \ln z_{2}\right)$ are actually Legendre transforms of each other). Their difference is a function of $c=\overline{z_{1}} z_{2}$ :

$$
\varphi_{\ell}^{E}\left(z_{1}, z_{2}\right)-\varphi_{1}^{E}\left(z_{1}\right) \equiv \mathfrak{R}(\mathbf{c})-\mathfrak{R}\left(\boldsymbol{\epsilon}_{0}^{E} \ln \mathbf{c}\right),
$$

whose real Hessian is the $2 \times 2$ matrix whose lines are respectively identified with $\bar{\epsilon}_{0}^{E} / \overline{\mathbf{c}}^{2}$ and $-i \bar{\epsilon}_{0}^{E} / \overline{\mathbf{c}}^{2}$. Its determinant is $-\left|\frac{\boldsymbol{\epsilon}_{0}^{E}}{\mathbf{c}^{2}}\right|^{2}$. If $E \neq 0$ then $\boldsymbol{\epsilon}_{0}^{E} \neq 0$ so the signature of that matrix is zero. This means that the value of Maslov's cocycle between $u_{\frac{\epsilon_{1 h}^{E}}{h}, \frac{\epsilon_{2 h}^{E}}{h}}$ and $\mathcal{F}_{h}^{-1} u_{\frac{-\epsilon_{1 h}^{E}}{h}, \frac{\epsilon_{2 h}^{E}}{h}}$ is zero.

The principal phases of those oscillatory functions are expressed by a formula similar to the one expressing the phase functions, but with a contribution of the sub-principal terms of $\epsilon_{i h}^{E}$ included. The two principal terms in the argument of $\left(c^{E}\right)^{\ell, 1}$ is their difference :

$$
\arg \left(c^{E}\right)^{\ell, 1} \equiv \frac{1}{h}\left(\mathfrak{R}\left(\boldsymbol{\epsilon}_{0}^{E}\right)-\mathfrak{R}\left(\boldsymbol{\epsilon}_{0}^{E} \ln \boldsymbol{\epsilon}_{0}^{E}\right)\right)-\mathfrak{R}\left(\boldsymbol{\epsilon}_{1}^{E} \ln \boldsymbol{\epsilon}_{0}^{E}\right)+O^{E}(h) .
$$

Here, we have fixed $E \neq 0$, so the $O^{E}(h)$ depends on $E$.

Finally, together with equation (21), we get that the first two terms in the expansion of $\left(C_{h}^{E}\right)^{\text {out }}$ are, for non zero $E$ : 


$$
\frac{1}{h}\left(\int_{\gamma_{1}^{E}} \alpha_{0}^{E}-\mathfrak{R}\left(\boldsymbol{\epsilon}_{0}^{E}\right)+\mathfrak{R}\left(\boldsymbol{\epsilon}_{0}^{E} \ln \boldsymbol{\epsilon}_{0}^{E}\right)\right)+\left(\int_{\gamma_{1}^{E}} \kappa^{E}+\mu\left(\gamma_{1}^{E}\right) \frac{\pi}{2}+\mathfrak{R}\left(\boldsymbol{\epsilon}_{1}^{E} \ln \boldsymbol{\epsilon}_{0}^{E}\right)\right),
$$

and the proof of proposition 6.14 is completed.

Of course, this also terminates the proof of theorem 6.9 .

\subsubsection{Recover the regular conditions}

The aim of this paragraph is to show how the quantization conditions of theorem 6.9 , when restricted to a non-singular Lagrangean, are equivalent to the regular quantization conditions of theorem 5.8.

Let us fix $E \neq 0$. We get a smooth holonomy 1 -form $\left[\lambda_{h}^{E}\right]$ whose action on a basis of the $H_{1}$ of the torus $\Lambda_{0}^{E}$ gives the two quantization conditions. Let $\gamma_{1}^{E}$ and $\gamma_{2}^{E}$ be, as previously, loops on $\Lambda_{0}^{E}$ representing such a basis.

The loop $\gamma_{2}^{E}$ can be assumed to lie in the neighborhood $\Omega$ where the normal form applies. In the linearized coordinates, one can choose $\gamma_{2}^{E}$ to be a simple orbit of the $\mathcal{X}_{2}$ vector field. On each point of that orbit we saw that the solutions were spanned by

$$
\frac{1}{r} e^{i \frac{\epsilon_{1}^{E}}{h} \ln r} e^{i \frac{\epsilon_{2}^{E}}{h} \theta}
$$

Therefore, the value of $\left[\lambda_{h}^{E}\right]$ on $\gamma_{2}^{E}$ is exactly the monodromy coefficient $\frac{2 \pi \epsilon_{2}^{E}(h)}{h}$. The quantization condition given by theorem 5.8 is thus

$$
\frac{2 \pi \epsilon_{2}^{E}}{h} \in 2 \pi \mathbb{Z}+O\left(h^{\infty}\right)
$$

which is indeed the same as the first singular quantization condition of theorem 6.9.

Remark 6.15. This simple observation gives rise, for non zero $E$, to an intrinsic definition of the semi-classical expansion $\epsilon_{2}^{E}(h)$ introduced in lemma 6.5 , namely :

$$
\epsilon_{2}^{E}(h)=\frac{1}{2 \pi} \int_{\gamma_{2}^{E}} \lambda^{E}(h) .
$$

Since $\epsilon_{2}^{E}$ is smooth at $E=0$, this implies that it is intrinsically defined by the microlocal system on a neighborhood of $m$, that is, independently of the Fourier integral operator $U$. This will be restated in the next paragraph.

Let us turn now to the second quantization condition. We know that the action of $\left[\lambda^{E}\right]$ on $\gamma_{1}^{E}$ is given by the argument of the quotient $\left(C^{E}\right)^{\text {out }} / C^{E}$. Using Stirling's formula for $\arg (\Gamma(z)) \equiv$ $\Im(\ln \Gamma(z))$, with $z=\frac{i \overline{\boldsymbol{\epsilon}}(h)}{2 h}+\frac{1}{2}\left(\boldsymbol{\epsilon}(h)=\epsilon_{1}^{E}(h)+i \epsilon_{2}^{E}(h)\right.$; recall that if $E \neq 0$, the principal term $\boldsymbol{\epsilon}_{0}$ of $\boldsymbol{\epsilon}(h)$ is non-zero), one can perform a very nice cancellation of undesirable terms in the local holonomy $C^{E}$. 
Indeed, one finds that

$$
2 \arg (\Gamma(z)) \equiv \frac{1}{h}\left(\mathfrak{R}(\boldsymbol{\epsilon} \ln \boldsymbol{\epsilon})-\mathfrak{R}(\boldsymbol{\epsilon})+\mathfrak{I}(\boldsymbol{\epsilon}) \frac{\pi}{2}-\mathfrak{R}(\boldsymbol{\epsilon} \ln (2 h))\right)+O(h) .
$$

Here of course, $O(h)$ depends on $E$. This gives

$$
\arg \left(C^{E}\right) \equiv \frac{1}{h}(\mathfrak{R}(\boldsymbol{\epsilon} \ln \boldsymbol{\epsilon})-\mathfrak{R}(\boldsymbol{\epsilon}))+O(h),
$$

that is,

$$
\arg \left(C^{E}\right) \equiv \frac{1}{h} \mathfrak{R}\left(\boldsymbol{\epsilon}_{0} \ln \boldsymbol{\epsilon}_{0}-\boldsymbol{\epsilon}_{0}\right)+\mathfrak{R}\left(\boldsymbol{\epsilon}_{1} \ln \boldsymbol{\epsilon}_{0}\right)+O(h) .
$$

We thus find that, if $E \neq 0$,

$$
\int_{\gamma_{1}^{E}} \lambda^{E}(h)=\frac{1}{h} \int_{\gamma_{1}^{E}} \alpha_{0}^{E}+\int_{\gamma_{1}^{E}} \kappa^{E}+\mu\left(\gamma_{1}^{E}\right) \frac{\pi}{2}+O(h),
$$

which gives the regular quantization condition of theorem 5.8.

\section{Structure of the joint spectrum}

This section is devoted to the application of our microlocal study to the precise study of the joint spectrum of 2-degree of freedom quantum integrable systems around the critical value of a focus-focus singularity.

More precisely, we shall assume in all this section that we are given two commuting essentially self-adjoint $h$-pseudo-differential operators of order zero $P_{1}(h), P_{2}(h)$ on a 2-dimensional manifold $X$, with the following properties:

- the joint principal symbol $p=\left(p_{1}, p_{2}\right)$ is proper with connected leaves;

- $p$ admits a critical point $m$ of focus-focus type, with critical value $0 \in \mathbb{R}^{2}$, such that the critical set $\Lambda_{0}=p^{-1}(0)$ has $m$ as its only critical point;

- at least one of the $P_{j}(h)$ 's is a classical elliptic pseudo-differential operator in the sense of Hörmander (for instance, $P_{1}(h)$ is a Schrödinger operator :

$$
P_{1}(h)=\frac{h^{2}}{2} \Delta_{g}+V(x),
$$

for some Riemannian metric $g$ on $X)$.

The last point implies that the solutions $u_{h}$ of $P_{j}(h) u_{h}=0(j=1,2)$ cannot have a wave front set at infinity (see [10]), which allows us to microlocalize $u_{h}$ on the Lagrangean level set $\Lambda_{0}=p^{-1}(0)$.

Note also that $P_{1}(h)$ and $P_{2}(h)$ really commute (and not only modulo $O\left(h^{\infty}\right)$ ). This assumption is perhaps unnecessary, but we take it in order to use the definition of the joint spectrum from $[5]$.

A typical example of this situation would be a Schrödinger operator on a surface of revolution with a radial potential, like the quantum spherical pendulum [15] or the quantum Champagne bottle [7]. As a matter of fact, we will illustrate our results with the latter example, thanks to numerics kindly provided by M.Child. 


\subsection{The joint spectrum}

Let $\Sigma(h)$ be the joint spectrum of the operators $P_{1}(h)$ and $P_{2}(h)$. We know from [5] that the assumption of properness for the momentum map $p$ implies that the intersection of $\Sigma(h)$ with any compact $K \subset \mathbb{R}^{n}$ is a discrete spectrum : each $E(h)=\left(E_{1}(h), E_{2}(h)\right) \in \Sigma(h) \cap K$ is isolated and the joint eigenspace

$$
F_{E(h)}=\left\{\varphi(h) \in L^{2}(X),\left(P_{1}(h)-E_{1}(h)\right) \varphi(h)=\left(P_{2}(h)-E_{2}(h)\right) \varphi(h)=0\right\}
$$

has finite dimension.

Theorem 7.1 There is a compact neighborhood $K$ of $0 \in \mathbb{R}^{n}$ and an $h_{0}>0$ such that all the joint eigenvalues $E(h) \in \Sigma(h) \cap K$ for $h<h_{0}$ are simple. Furthermore, the distance between two distinct joint eigenvalues is bounded below by some finite power of $h$.

Proof. This is a consequence of proposition 6.4, applied to the operators $P_{j}^{E}(h) \stackrel{\text { def }}{=} P_{j}(h)-E_{j}$. Indeed, let $K \subset \subset \mathbb{R}^{n}$ be a neighborhood of zero such that this proposition holds uniformly for $E=\left(E_{1}, E_{2}\right) \in K$. Let $\psi_{1}(h)$ and $\psi_{2}(h)$ be two normalized joint eigenfunctions for the joint eigenvalue $E(h)$, such that $\left\langle\psi_{1}, \psi_{2}\right\rangle=0$. If we can find such eigenfunctions for a set of values of $h$ accumulating at zero, then $\psi_{1}(h)$ and $\psi_{2}(h)$ are both microlocal solutions (see section 4.5) to the equation

$$
\left(P_{j}(h)-E_{j}(h)\right) \psi_{k}(h) \sim 0, \quad j=1,2 \quad k=1,2 .
$$

Therefore, their wave front sets are included in a compact $W \subset T^{*} X$. Let $\pi: T^{*} X \rightarrow X$ be the standard projection and let $U$ be an open neighborhood of $\pi(W)$ such that $\bar{U}$ is compact. Then,

$$
W F_{h}\left(\psi_{k}\right) \cap\left(T^{*}(X \backslash U)\right)=\emptyset \quad(k=1,2),
$$

and because $\psi_{k}$ cannot have any wave front set at infinity, $\psi_{k}$ is actually $O\left(h^{\infty}\right)$ on $X \backslash U$. Consequently if we let $\chi$ be a smooth function with support in $U$ and equal to 1 on $\pi(W)$ then

$$
\begin{gathered}
\left\|\chi \psi_{k}\right\|_{L^{2}(X)}=\left\|\psi_{k}\right\|_{L^{2}(X)}+O\left(h^{\infty}\right)=1+O\left(h^{\infty}\right), \\
\text { and }\left\langle\chi \psi_{1}, \chi \psi_{2}\right\rangle=O\left(h^{\infty}\right) .
\end{gathered}
$$

That allows us to replace, in the following, $\psi_{k}$ by $\chi \psi_{k}$.

Because of proposition 6.4 , there exists a constant $C(h) \in \mathbb{C}_{h}$ such that

$$
\psi_{1}(h) \sim C(h) \psi_{2}(h) \text { on } T^{*} U
$$

Combined as before with the fact that nothing happens at infinity, equation (22) implies, by taking scalar products, that $C(h)$ simultaneously satisfies $|C(h)|=1+O\left(h^{\infty}\right)$ and $C(h)=$ $0+O\left(h^{\infty}\right)$, which is impossible. This proves that for small $h$ all joint eigenvalues $E(h)$ must be simple.

Now, if $E(h)$ and $E^{\prime}(h)$ are distinct joint eigenvalues but differing only by $O\left(h^{\infty}\right)$, the same argument applies to the eigenfunctions $\psi_{1}(h)$ and $\psi_{2}(h)$ associated to $E(h)$ and $E^{\prime}(h)$, and thus proves the last statement of the theorem. 
This theorem extends the results of [5], where it is shown that the eigenvalues are simple as long as $K$ does not meet any critical value of the momentum map $p$. Note that this latter statement comes as a consequence of our proposition 5.1 as well.

Finally, remark that the proof of theorem 7.1 actually shows that joint eigenfunctions $\psi(h)$ corresponding to joint eigenvalues $E(h) \in K$ are in one-to-one correspondence with microlocal solutions of the system

$$
\left(P_{j}(h)-E_{j}(h)\right) u_{h} \sim 0
$$

in a neighborhood of $\bigcup_{E \in K} \Lambda_{E}$. In other words, these eigenfunctions are determined by the quantization conditions of theorem 6.9.

\subsection{Quantum monodromy}

Away from the critical value of the momentum map $p$, the joint spectrum locally looks like a lattice $h \mathbb{Z}^{2}$. However, simply patching these lattices together around 0 is not possible. This phenomenon is called quantum monodromy and is described in details in [40]. Let us take advantage of section 5 to give a short description of it.

If $c_{0}$ is regular value of $p$, then on a neighborhood $B$ of $c_{0}$ there exists a map $f(h): B \rightarrow$ $\mathbb{R}^{2}$ that "asymptotically identifies $\Sigma(h) \cap B$ with the straight lattice $h \mathbb{Z}^{2}$ ", in the following sense : $f(h)$ is an elliptic semi-classical symbol of order zero such that for any family $E(h)=$ $\left(E_{1}(h), E_{2}(h)\right) \in B, h \in H$

$$
E(h) \in \Sigma(h) \cap B+O\left(h^{\infty}\right) \text { if and only if } f(E(h) ; h) \in h \mathbb{Z}^{2}+O\left(h^{\infty}\right) .
$$

Indeed, for $c \in B$ let $\lambda_{c}(h)$ be the semi-classical holonomy 1-form on $\Lambda_{c}$ defined in proposition 5.5 ; then because of theorem 5.8 , one can choose

$$
f(c ; h) \stackrel{\text { def }}{=}\left(\frac{1}{2 \pi} \int_{\gamma_{1}(c)} \lambda_{c}(h), \frac{1}{2 \pi} \int_{\gamma_{2}(c)} \lambda_{c}(h)\right),
$$

where $\left(\gamma_{1}(c), \gamma_{2}(c)\right)$ is any smooth basis of $H_{1}\left(\Lambda_{c}\right)$.

It turns out that up to conjugation of $f(h) / h$ by an element of the affine group with integer coefficients $G A(2, \mathbb{Z})$ such a map $f(h)$ is unique modulo $O\left(h^{\infty}\right)$. If $\delta$ is a simple loop through $c_{0}$ enclosing the critical value 0 and small enough not to enclose any other critical value of the momentum map, we can cover its image by a finite number of open ball $B_{0}, \ldots, B_{\ell}$. Assuming that $B_{0}=B$, we can construct a corresponding map $f_{1}(h)$ on $B_{1}$ that coincide with $f(h)$ on $B_{0} \cap B_{1}$. Continuing this way, we end up with a map $f_{\ell}(h)$ on $B_{\ell}$, and an element $\mu(\delta) \in G A(2, \mathbb{Z})$ such that

$$
\frac{1}{h} f_{\ell}(h)=\mu(\delta) \circ\left(\frac{1}{h} f(h)\right)+O\left(h^{\infty}\right) .
$$

$\mu(\delta)$ depends only on the homotopy class of $\delta$ and is called the quantum monodromy of the joint spectrum. Because the $f_{j}(h)$ 's can be chosen as in equation $(24)$, this $\mu(\delta)$ is actually dual to the classical monodromy of the bundle $\pi_{1}\left(\Lambda_{c}\right) \rightarrow c$ : 
Proposition $7.2([\mathbf{4 0}])$ If $\left(\gamma_{1}(c), \gamma_{2}(c)\right)$ and $\epsilon$ are chosen as in proposition 6.3 then

$$
\mu(\delta)=\iota\left(\begin{array}{cc}
1 & -\epsilon \\
0 & 1
\end{array}\right)
$$

where $\iota$ is the inclusion $G L(2, \mathbb{R}) \hookrightarrow G A(2, \mathbb{R})$ such that for any $M \in G L(2, \mathbb{R})$, the origin $0 \in \mathbb{R}^{2}$ is fixed by $\iota(M)$.

The article [40] shows how this quantum monodromy can be told from a picture of the spectrum by detecting the change in the lattice structure of the joint spectrum around the critical value. One of the ways of doing this is to unwind the spectrum onto $\mathbb{R}^{2}$, in the following way. Suppose $\delta$ is actually drawn on the universal cover of the punctured plane : $\tilde{W} \stackrel{\pi}{\longrightarrow} \mathbb{R}^{2} \backslash\{0\}$ and let $\tilde{B}_{j}$ be the open cover of its image such that $\pi\left(\tilde{B}_{j}\right)=B_{j}$. Then the maps $f_{j}(h)$ on $B_{j}$ define a global map $\hat{f}(h)$ on $\tilde{B}_{0} \cup \tilde{B}_{1} \cdots \cup \tilde{B}_{\ell}$. By definition we have

$$
\hat{f}(\delta(1) ; h) / h=\mu(\delta) \circ \hat{f}(\delta(0) ; h) / h .
$$

Since the restriction of $\hat{f}(h)$ to the points belonging to the spectrum $\pi^{-1}(\Sigma(h))$ takes values in $h \mathbb{Z}^{2}+O\left(h^{\infty}\right)$, there is for small $h$ a unique map

$$
\tilde{f}(h): \pi^{-1}(\Sigma(h)) \cap \bigcup_{j} \tilde{B}_{j} \rightarrow h \mathbb{Z}^{2}
$$

that coincides with $\hat{f}(h)$ modulo $O\left(h^{\infty}\right)$. This map is of course as well defined on the intersection with $\pi^{-1}(\Sigma(h))$ of any domain $\tilde{\mathcal{D}}$ of $\tilde{W}$ that projects into a small (but fixed) annulus $\mathcal{D}$ around the critical value 0 , and is called the unwinding (or developing) map of the spectrum.

For instance, if one uses this map to unwind a sequence of points $\tilde{A}_{j}(h) \in \pi^{-1}(\Sigma(h)) \cap \tilde{B}_{j}$ with $\pi\left(\tilde{A}_{\ell}(h)\right)=\pi\left(\tilde{A}_{0}(h)\right)$ (see figure 5 ), then one obtains an integral open polygonal line $\left(A_{0}, \ldots, A_{\ell}\right)$ in $h \mathbb{Z}^{2}$, whose extremities satisfy

$$
A_{\ell} / h=\mu(\delta)\left(A_{0} / h\right)
$$

For short, we shall allow us to refer to such a sequence of points $\tilde{A}_{j}$ as a closed polygonal line in $\Sigma(h)$ whenever these points are close enough to each other so that the line segments $\left[A_{j}, A_{j+1}\right]$ lie in $\hat{f}(\tilde{\mathcal{D}} ; h)$. The "segment" between two consecutive points $\tilde{A}_{j}(h)$ and $\tilde{A}_{j+1}(h)$ is by definition the preimage in $\tilde{\mathcal{D}}$ of $\left[A_{j}, A_{j+1}\right]$.

\subsection{The exact counting function}

The purpose of this paragraph is to count the number of joint eigenvalues inside a simple closed polygonal line around the critical value 0 . Let $L_{0}(h)$ be the subset of $\Sigma(h) \cap \mathcal{D}$ of joint eigenvalues $E(h)$ that are unwound on the horizontal line $\{y=0\} \subset \mathbb{R}^{2}$ (in other words, $\left.\tilde{f}_{2}\left(\pi^{-1}(E(h)) ; h\right)=0\right)$. Because of proposition $7.2, L_{0}(h)$ is pointwise fixed by the quantum monodromy $\mu(\delta)$. That means that any closed polygonal line $\tilde{\mathcal{A}}(h)=\left(\tilde{A}_{0}(h), \ldots, \tilde{A}_{\ell}(h)\right)$ on $\Sigma(h)$ starting on $L_{0}(h)$ is unwound as a closed polygonal line $\mathcal{A}(h)=\left(A_{0}(h), \ldots, A_{\ell}(h)\right)$ in $\mathbb{R}^{2}$ (see figure 5).

It is easy to see that if we have another such polygonal line on $\Sigma(h) \cap \mathcal{D}$ that lies inside the previous one, then the number of joint eigenvalue between these two is exactly the number of 
a)
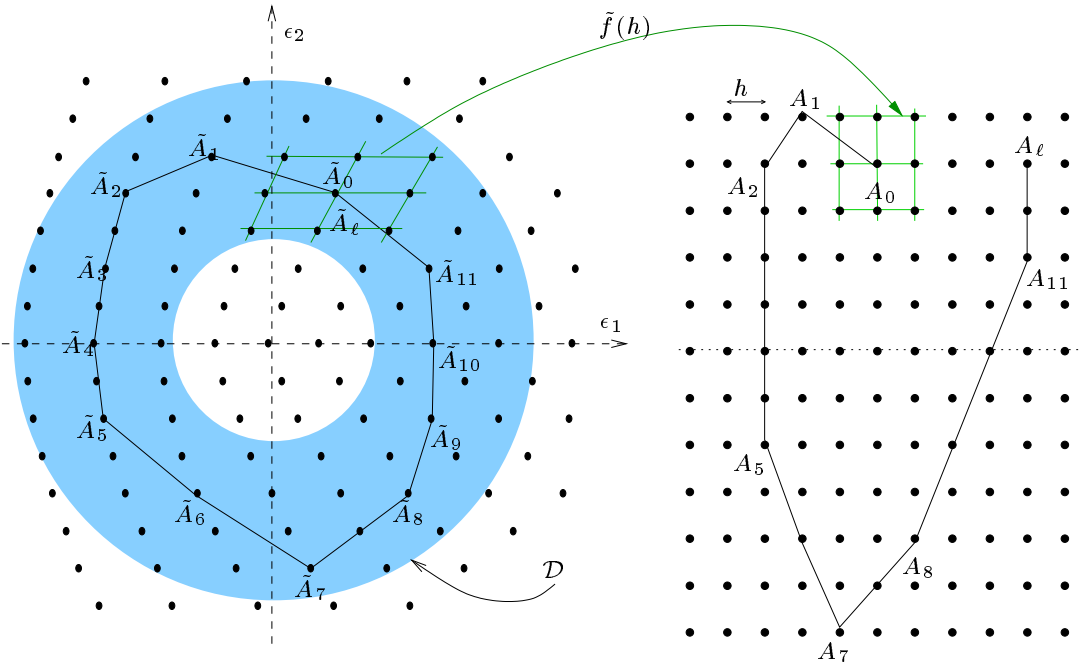

b)

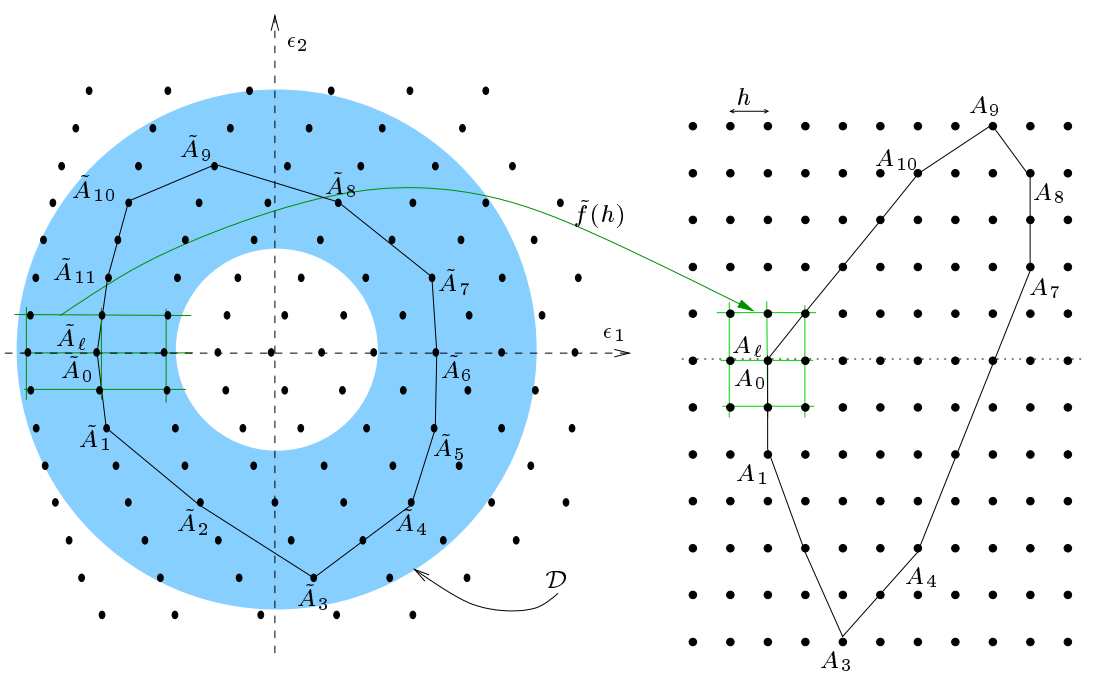

Figure 5: unwinding of a sequence of points in $\Sigma(h) \cap \mathcal{D}$, in the case of the spectrum of the Champagne bottle (numerics by M.Child). In the case $b$ ), the starting point belongs to $L_{0}(h)$, which implies that the unwound line is closed. 
$h$-integral lattice points between the two unwound polygons (see lemma 7.4). As a consequence, there should be a universal index $\nu \in \mathbb{Z}$ such that the number $N_{h}(\tilde{\mathcal{A}}(h))$ of joint eigenvalues inside $\tilde{\mathcal{A}}(h)$ be

$$
N_{h}(\tilde{\mathcal{A}}(h))=N_{h}(\mathcal{A}(h))+\nu
$$

where $N_{h}(\mathcal{A}(h))$ denotes the number of $h$-integral points inside the polygon $A(h)$. Note that the latter is given by Pick's formula [36] :

$$
N_{h}(\mathcal{A}(h))=\operatorname{Area}(\mathcal{A}(h))+\frac{\mathbb{Z} \text { Length }(\mathcal{A}(h))}{2}+1,
$$

where $\mathbb{Z}$ Length $(\mathcal{A}(h))$ is the number of $h$-integral points on the boundary.

The result is actually that $\nu=0$ :

Theorem 7.3 Let $\tilde{\mathcal{A}}(h)=\left(\tilde{A}_{0}(h), \ldots, \tilde{A}_{\ell}(h)\right)$ be a simple closed polygonal line on $\Sigma(h)$ starting on $L_{0}(h)$ and enclosing one the origin, and let $\mathcal{A}(h)=\left(A_{0}(h), \ldots, A_{\ell}(h)=A_{0}(h)\right)$ be the unwound polygonal line in $\mathbb{R}^{2}$. Denote by $N_{h}(\tilde{\mathcal{A}}(h))$ the number of joint eigenvalues inside $\tilde{\mathcal{A}}(h)$ (counting the points in the boundary), and similarly denote by $N_{h}(\mathcal{A}(h))$ the number of $h$-integral points inside the polygon $A(h)$, counting the boundary.

Then

$$
N_{h}(\tilde{\mathcal{A}}(h))=N_{h}(\mathcal{A}(h)) .
$$

In other words, the recipe to calculate $N_{h}(\tilde{\mathcal{A}}(h))$ is simply to unwind $\tilde{\mathcal{A}}(h)$ and then apply Pick's formula (25).

Proof. The idea is to cut the "polytope" delimited by $\tilde{\mathcal{A}}(h)$ into two halves separated by $L_{0}$; then on each part the control over the joint eigenvalues given by theorem 6.9 is used to shift the problem into a region where the result will be obvious, due to the following fact :

Lemma 7.4 if $B$ is a simply connected open subset of $\mathcal{D}$, then for any closed polygonal line $\tilde{\mathcal{C}}(h)$ in $\Sigma(h) \cap B$, the unwound line $\mathcal{C}(h)$ is also closed and we have

$$
N_{h}(\tilde{\mathcal{C}}(h))=N_{h}(\mathcal{C}(h))
$$

This lemma also proves the existence of the index $\nu$ claimed before the statement of the theorem, and this implies that we can choose any particular $\tilde{\mathcal{A}}(h)$ to prove the theorem. So let us assume that the intersection of the polytope delimited by $\tilde{\mathcal{A}}(h)$ with the horizontal lines $\epsilon_{2}=h n$ are segments whose extremities are vertices of $\tilde{\mathcal{A}}$.

Now, let us apply theorem 6.9 to the operators $P_{j}^{E}(h) \stackrel{\text { def }}{=} P_{j}(h)-E_{j}$, and keep the notations from the statement of this theorem. We know from lemma 6.5 that the map

$$
E=\left(E_{1}, E_{2}\right) \rightarrow \epsilon^{E}(h)=\left(\epsilon_{1}^{E}(h), \epsilon_{2}^{E}(h)\right)
$$

is an elliptic semi-classical symbol of order zero. It is therefore for small $h$ a diffeomorphism of a compact neighborhood $K$ of zero into its image; this implies that the determination of the 
joint spectrum in $K$ amounts to that of the corresponding values of $\boldsymbol{\epsilon}^{E}(h)$, which we shall hence simply denote by $\boldsymbol{\epsilon}=\left(\epsilon_{1}, \epsilon_{2}\right)$. From now on, we shall assume that the annulus $\mathcal{D}$ defined in the previous paragraph is a subset of $K$.

Let us look for solutions satisfying $\epsilon_{2} \geqslant 0$. Let $g_{1}(h)$ and $g_{2}(h)$ be the following functions:

$$
\begin{gathered}
\frac{2 \pi}{h} g_{1}(\epsilon ; h)=\left(\lambda^{E}\right)^{\text {out }}+\epsilon_{2} \frac{\pi}{2}-\frac{\epsilon_{1}}{h} \ln (2 h)-2 \arg \Gamma\left(\frac{i \epsilon_{1} / h+1+\epsilon_{2}}{2}\right), \\
\text { and } g_{2}(\epsilon ; h)=\epsilon_{2} .
\end{gathered}
$$

Because of the analyticity properties of $\Gamma$, these functions are holomorphic in $\epsilon_{2} \geqslant 0$. The map $f(E ; h) \stackrel{\text { def }}{=} g\left(\epsilon^{E}(h) ; h\right)$ satisfies equation $(23)$ thanks to theorem 6.9. Because of the $\ln h$ term in it, it is not a standard semi-classical symbol globally; but we know from paragraph 6.7.4 that it is so for $E$ in a small compact at finite distance from the origin. Moreover, because of remark 6.15 , it must satisfy equation (24) as well, where $\left(\gamma_{1}(c), \gamma_{2}(c)\right)$ is the basis given in proposition 6.3 .

Therefore, the joint spectrum is spread on the horizontal lines $\epsilon_{2}=h n, n \in \mathbb{Z}$, and the set $L_{0}(h)$ lies in the line $\epsilon_{2}=0$. Our problem is now to count the number of joint eigenvalues inside the intersection $\tilde{\mathcal{A}}^{+}(h)$ of the half plane $\epsilon_{2} \geqslant 0$ with the polytope delimited by $\tilde{\mathcal{A}}(h)$ (figure 6 ). Let us denote by $\tilde{I}_{n}(h)$ the set of joint eigenvalues contained in $\tilde{\mathcal{A}}^{+}(h)$ and satisfying $\epsilon_{2}=h n$.

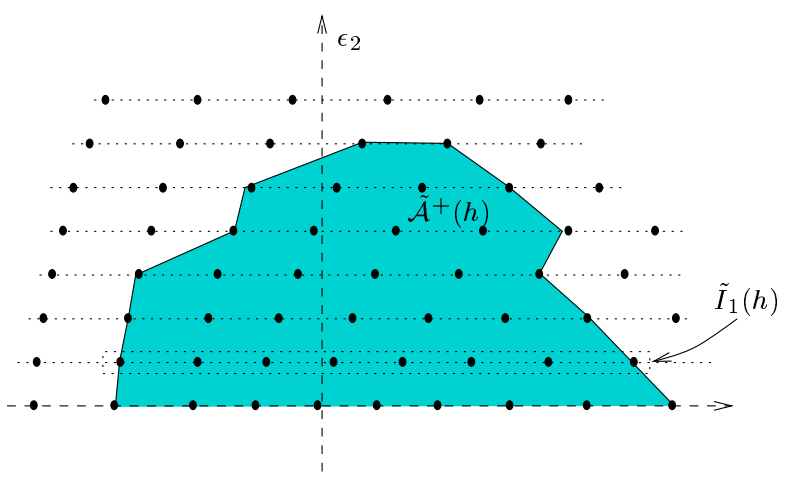

Figure 6: the upper half of the polytope.

From now on, we forget the negative half of our polytope, and label

$$
\tilde{A}_{0}, \ldots, \tilde{A}_{\ell}
$$

the consecutive vertices of $\tilde{\mathcal{A}}^{+}(h)$, starting and ending on $L_{0}(h)$. We denote by $N_{h}(\tilde{\mathcal{A}}(h))$ the number of joint eigenvalues inside $\tilde{\mathcal{A}}^{+}(h)$, and by $N_{h}(\mathcal{A}(h))$ the number of $h$-integral points inside the polytope delimited by the unwound points $A_{0}, \ldots, A_{\ell}, A_{0}$. The theorem will be proved provided we show that $N_{h}(\tilde{\mathcal{A}}(h))=N_{h}(\mathcal{A}(h))$.

The next step of the proof is now to translate $\tilde{\mathcal{A}}^{+}(h)$ far from the origin, as follows (figure 7 ). The domain $\mathcal{D}$ where the unwinding map is defined can always be chosen such that there exists 
an integer $k(h) \in \mathbb{Z}$ such that the origin is outside of the polytope $\tilde{\mathcal{C}}(h)=\left(\tilde{C}_{0}(h), \ldots, \tilde{C}_{\ell}(h)\right)$ defined by

$$
\tilde{f}\left(\tilde{C}_{j}(h) ; h\right)=\tilde{f}\left(\tilde{A}_{j}(h) ; h\right)+h(k(h), 0) .
$$

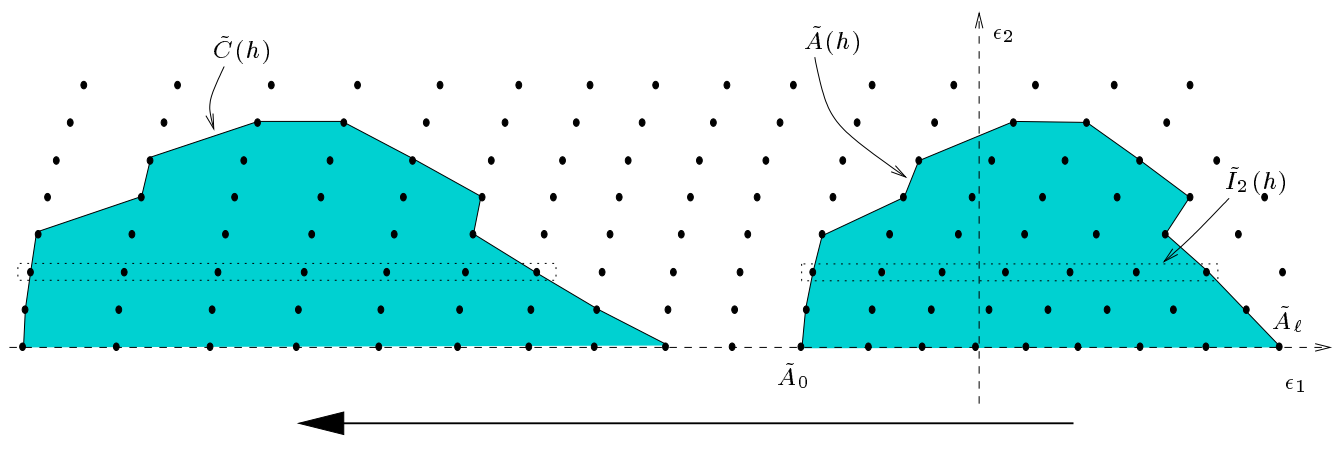

Figure 7: translation of the polytope

Of course, the set of joint eigenvalues inside $\tilde{\mathcal{C}}(h)$ satisfying $\epsilon_{2}=h n$ is in bijection with $\tilde{I}_{n}(h)$, which implies that

$$
N_{h}(\tilde{\mathcal{C}}(h))=N_{h}(\tilde{\mathcal{A}}(h))
$$

On the other hand, because $f(\cdot ; h)$ is a valid unwinding function in a region containing both $\tilde{\mathcal{A}}(h)$ and $\tilde{\mathcal{C}}(h)$, the unwinding $\mathcal{C}(h)$ of $\tilde{\mathcal{C}}(h)$ is by definition the translation of $\mathcal{A}(h)$ by the horizontal vector $h(k(h), 0)$. It has therefore the same number of interior points :

$$
N_{h}(\mathcal{C}(h))=N_{h}(\mathcal{A}(h))
$$

But lemma 7.4 yields

$$
N_{h}(\tilde{\mathcal{C}}(h))=N_{h}(\mathcal{C}(h))
$$

which finally proves the theorem.

\subsection{Shape of the spectrum near the critical value}

We investigate here the shape of the joint spectrum in a region of size $O(h)$ near the origin, in terms of the $\left(\epsilon_{1}, \epsilon_{2}\right)$ variables. We already know that the joint eigenvalues are distributed on the horizontal lines $\epsilon_{2}=h n, n \in \mathbb{Z}$. It remains to study their repartition on each of these lines. For this purpose, we fix $n \in \mathbb{Z}$ and denote by $x=\epsilon_{1} / h$ the generic variable on $\left\{\epsilon_{2}=h n\right\}$. Of course, the results will be based upon corollary 6.10 , whose notations we use here, and which we express in the following way : 
Proposition 7.5 (corollary 6.10) Let $K$ a compact neighborhood of the origin in $\mathbb{R}^{2}$. For any family $E(h)=\left(E_{1}(h), E_{2}(h)\right) \in(h K)$ such that $\epsilon_{2}^{E(h)}(h)=h n+O\left(h^{\infty}\right)(n \in \mathbb{Z})$, the following characterization holds :

$$
\begin{gathered}
E(h) \in \Sigma(h)+O\left(h^{\infty}\right) \quad \text { if and only if } \\
|n| \frac{\pi}{2}-x(h) \ln (2 h)-2 \arg \Gamma\left(\frac{i x(h)+1+|n|}{2}\right)+f_{n}(x(h) ; h) \in 2 \pi \mathbb{Z}+O\left(h^{\infty}\right),
\end{gathered}
$$

with $x(h)=\epsilon_{1}^{E(h)}(h) / h$. Here $f_{n}(h)$ is a symbol of the form :

$$
f_{n}(x ; h)=\frac{A}{h}+x B+n C+D+\mu \frac{\pi}{2}+h f_{n, 1}(x)+h^{2} f_{n, 2}(x)+\cdots,
$$

for some real constants $A, B, C, D$.

Proof. Of course, in view of corollary 6.10 - applied to the system $\tilde{P}_{j}^{t}=P_{j}-h t_{j}, j=1,2-, A$ is just the action integral on the critical Lagrangean : $A=\int_{\gamma_{1}^{0}} \alpha_{0}$, and it only remains to prove that there exists constants $B, C, D$ such that

$$
I_{\gamma_{1}^{0}}\left(\tilde{\kappa}^{t}\right)=x(h) B+n C+D+O(h),
$$

where $t$ and $x(h)$ are linked by

$$
x(h)=\tilde{\epsilon}_{1}^{t}(h)=\frac{1}{h} \epsilon_{1}^{h t}(h) .
$$

Because the sub-principal symbols of $\tilde{P}_{j}^{t}$ are affine functions of $t: \tilde{r}^{t}=r-t$, it is easy to deduce from formula (17) of proposition 6.8 that $I_{\gamma_{1}^{0}}\left(\tilde{\kappa}^{t}\right)$ is affine in $t$. The result then follows from the fact that the principal part of $\tilde{\boldsymbol{\epsilon}}^{t}(h)=\left(x(h), n+O\left(h^{\infty}\right)\right)$ is also an - invertible - affine function of $t$.

We study the distribution of joint eigenvalues on the line $\epsilon_{2}=h n$ by investigating the gaps between two consecutive eigenvalues.

Theorem 7.6 For any $n \in \mathbb{Z}$, let $E_{k}(h)$ be the sequence of joint eigenvalues in $\left\{\epsilon_{2}=h n\right\} \cap(h K)$, such that the corresponding variables $x_{k}(h)$ form (for each $h$ ) a strictly increasing finite sequence.

The gap between $E_{k}(h)$ and $E_{k+1}(h)$ is given by:

$$
\begin{aligned}
& \left\|\frac{E_{k+1}(h)-E_{k}(h)}{h}\right\|=\frac{2 \pi a}{|\ln h|+B-\ln 2-\Psi_{n}^{\prime}(x)}(1+O(h)) \\
& =a\left(\frac{2 \pi}{|\ln h|}+\frac{2 \pi}{(\ln h)^{2}}\left(\Psi_{n}^{\prime}(x)-B+\ln 2\right)\right)+O\left(\frac{1}{(\ln h)^{3}}\right),
\end{aligned}
$$

for an $x \in] x_{k}, x_{k+1}[$, and $a=\|M \cdot(1,0)\|$; we have denoted

$$
\Psi_{n}(x)=2 \arg \Gamma\left(\frac{i x+1+|n|}{2}\right) .
$$


Note that $\Psi_{n}^{\prime}$ is related to the logarithmic derivative $\psi$ of the Gamma-function :

$$
\Psi_{n}^{\prime}(x)=\Re\left(\psi\left(\frac{i x+1+n}{2}\right)\right)
$$

A consequence of this theorem is that the number of $E_{k}(h)$ 's inside $h K$ increases as $h$ tends to zero at a rate equivalent to const.| $\ln h \mid$, which easily yields a Weyl type asymptotic formula for the counting function (see theorem 7.7 in next paragraph). A more precise result is that the function $\Psi_{n}^{\prime}$ controls the shape of the graph "gaps versus $x$ ". It turns out that $\Psi_{n}^{\prime}$ has a non-degenerate minimum at $x=0$, which is particularly sharp for $n=0$ (figure 8 ). This might

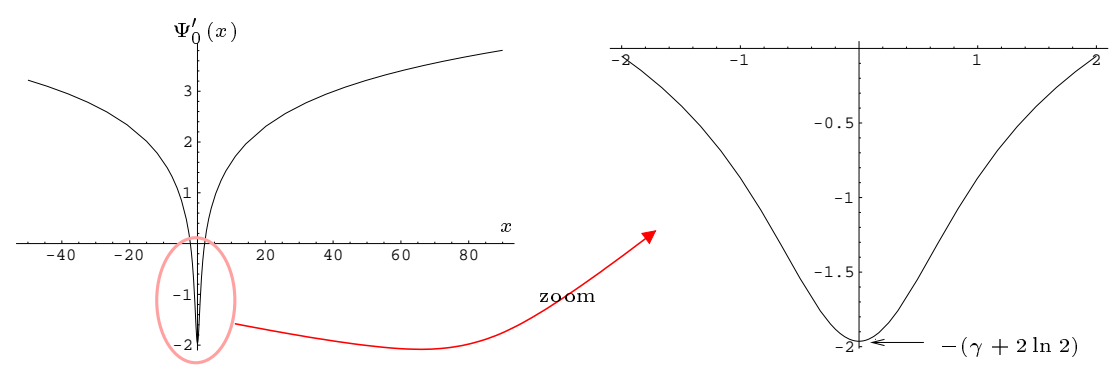

Figure 8: the function $\Psi_{0}^{\prime}(x)$ (here $\gamma$ denotes Euler's constant $\gamma \simeq 0,5772156649 \ldots$ ).

have some interesting applications in numerical analysis. First, it should refrain people from thinking that the gap curve has a cusp; secondly, however, this "near-cusp" might be useful to precisely locate the critical value $x=0$. Numerics for the example of the Champagne bottle suggest that both remarks apply for reasonable value of the small constant $h$ (figures 9 and 10).

Finally, of course, this theorem can be used to find a rigorous approximation for the spectral

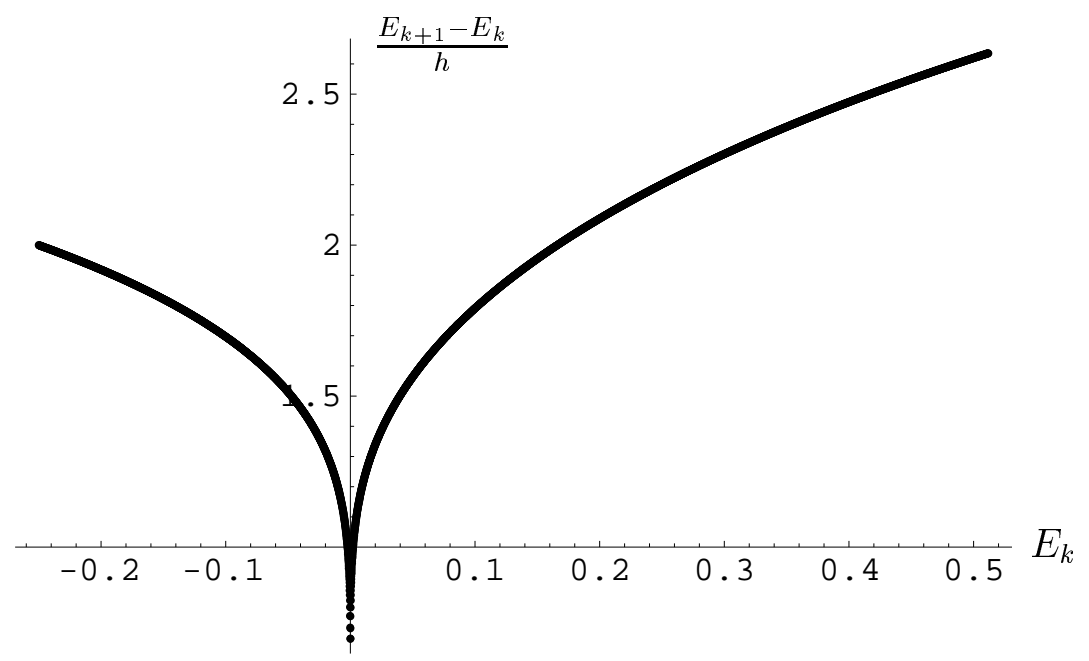

Figure 9: spectral gaps for the quantum Champagne bottle, $n=0, h=10^{-4}$ (numerics).

gaps. In the example of the Champagne bottle, formula (26) can be made completely explicit. 


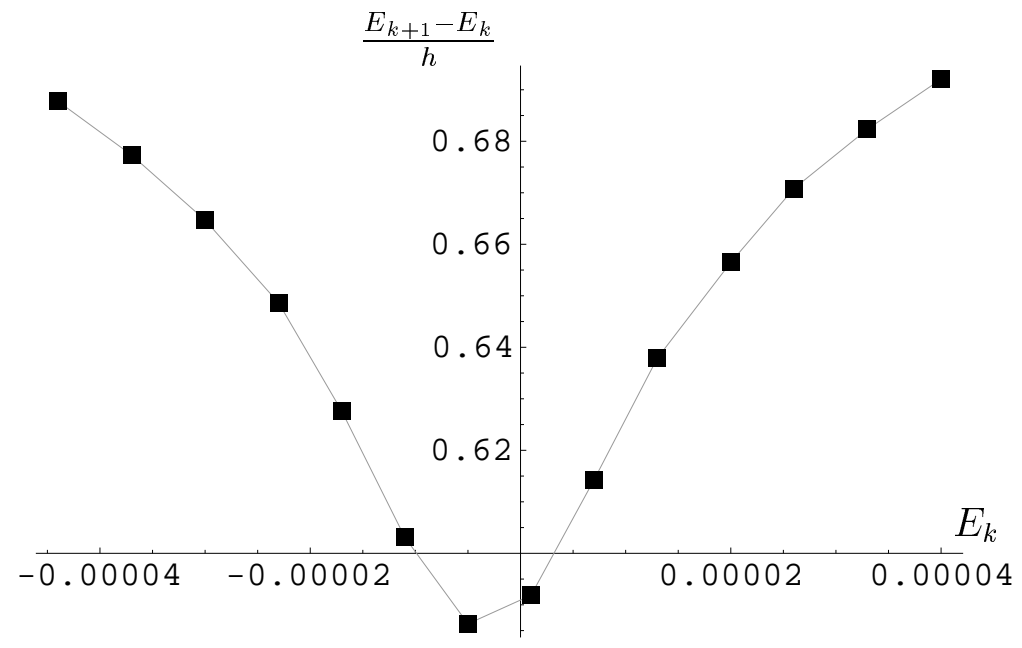

Figure 10: spectral gaps for the quantum Champagne bottle, $n=0, h=10^{-5}$ (numerics).

The Hamiltonian $H$ is the following :

$$
H(x, y, \xi, \eta)=\frac{1}{2}\left(\xi^{2}+\eta^{2}\right)-r^{2}+r^{4},
$$

with $r^{2}=x^{2}+y^{2}$. It commutes with the angular momentum $I=x \eta-y \xi$, hence we can let $p=\left(p_{1}, p_{2}\right)=(H, I)$. The corresponding $h$-operators are

$$
\hat{H}=-\frac{h^{2}}{2} \Delta-r^{2}+r^{4}, \quad \hat{I}=\frac{h}{i} \frac{\partial}{\partial \theta} .
$$

One can compute the quantities involved by formula (26), and one finds

$$
M=\left(\begin{array}{cc}
\sqrt{2} & 0 \\
0 & 1
\end{array}\right) \text { so } a=\sqrt{2}, \quad \text { and } B=(5 / 2) \ln 2 .
$$

Thus, using formula (26) to approximate the smallest gap (obtained at $x=0$ for $n=0$ ) we get :

$$
\min _{k}\left(\frac{E_{k+1}-E_{k}}{h}\right) \sim \frac{2 \pi \sqrt{2}}{|\ln h|+(9 / 2) \ln 2+\gamma}+O(h) .
$$

The comparison between the principal part of this formula as a function of $\ln h$ and the "experimental" result from the numerical computation of the spectrum is depicted in figure 11, and tends to show that the approximation is quite accurate.

Proof of theorem 7.6. Because $\left(E_{k+1}-E_{k}\right) / h=(M+O(h)) \cdot\left(x_{k+1}-x_{k}, 0\right)+O\left(h^{\infty}\right)$, it suffices to estimate $\left|x_{k+1}-x_{k}\right|$.

For $n \in \mathbb{Z}$, let

$$
g_{n}(x ; h) \stackrel{\text { def }}{=} \frac{1}{2 \pi}\left(|n| \frac{\pi}{2}-x \ln (2 h)-2 \arg \Gamma\left(\frac{i x+1+|n|}{2}\right)+f_{n}(x ; h)\right) .
$$

The function $x \rightarrow g_{n}(x ; h)$ is smooth and we have

$$
g_{n}^{\prime}(x ; h)=B-\ln (2 h)-\Psi_{n}^{\prime}(x)+O(h) .
$$




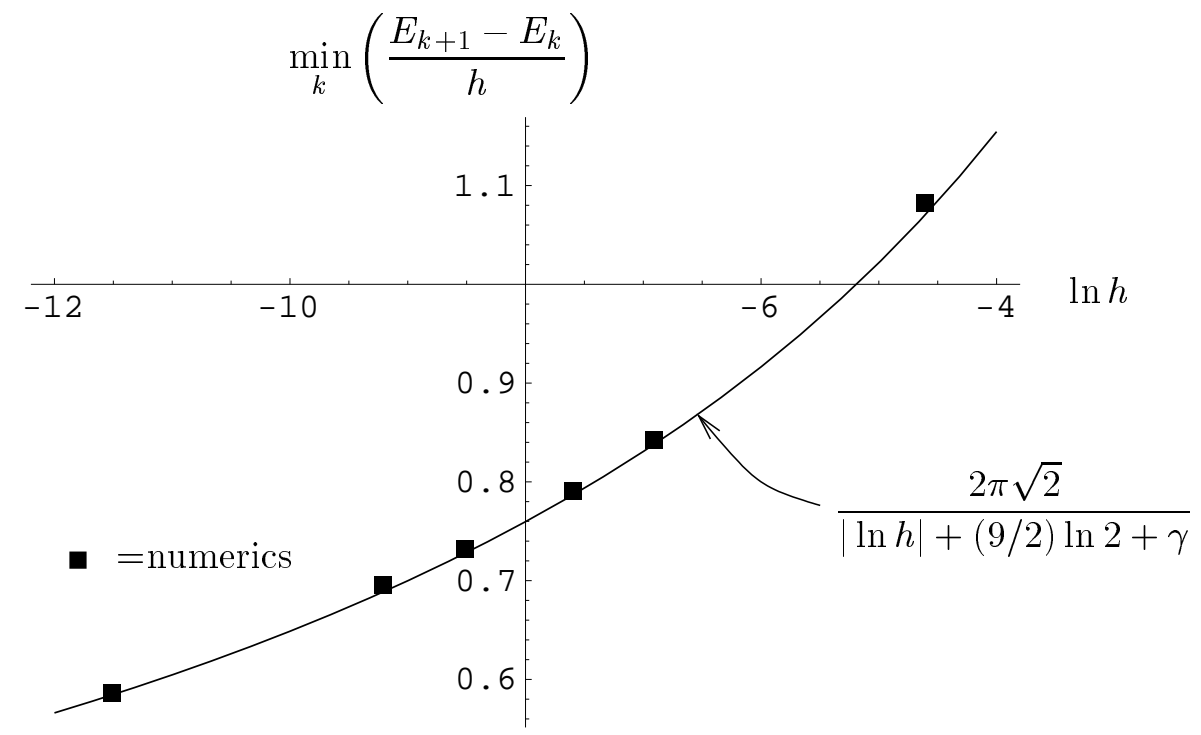

Figure 11: The smallest gap; comparison between numerics and the top order term in formula (26).

For small $h, g_{n}(h)$ is therefore strictly increasing, with slope of order $|\ln h|$. The solutions $x_{k}(h)$ to $g\left(x_{k}(h) ; h\right)=k \in \mathbb{Z}+O\left(h^{\infty}\right)$ are therefore, for small $h$, in one-to-one correspondence with the exact solutions to $g\left(x_{k}(h) ; h\right)=k$ (except maybe for the first and the last ones, sitting near the boundary of the compact interval in which we are looking for solutions). Moreover the differences between the exact and the $O\left(h^{\infty}\right)$ solutions are of order $\frac{1}{|\ln h|} O\left(h^{\infty}\right)$, which is $a$ fortiori $O\left(h^{\infty}\right)$. In the following $x_{k}$ denotes the exact solution.

By Rolle's theorem, there is an $x \in] x_{k}, x_{k+1}$ [ such that

$$
\frac{1}{\left|x_{k+1}-x_{k}\right|}=g_{n}^{\prime}(x ; h) \text {. }
$$

This means

$$
\left|x_{k+1}-x_{k}\right|=\frac{2 \pi}{|\ln h|+B-\ln 2-\Psi_{n}^{\prime}(x)+O(h)},
$$

which proves the theorem.

\subsection{Weyl's formula}

Let $K$ be a compact convex subset of $\mathbb{R}^{2}$, and let $\tilde{K}(h)$ be its image by the change of variables:

$$
\left(t_{1}, t_{2}\right) \rightarrow\left(\tilde{\epsilon}_{1}^{t}(h), \tilde{\epsilon}_{2}^{t}(h)\right) .
$$

Recall that the principal part of this map is

$$
\tilde{\boldsymbol{\epsilon}}_{0}^{t}=M^{-1} \cdot(t-r(m)),
$$

where $r(m)=\left(r_{1}(m), r_{2}(m)\right)$ is the value at $m$ of the joint sub-principal symbol of the system $P_{1}(h), P_{2}(h)$, and $M=d F(0)$, where $\left(p_{1}, p_{2}\right)=F\left(q_{1}, q_{2}\right)$ for some symplectic coordinates near $m$ (see section 6.5). 
Denote by $\tilde{K}_{0}$ the principal part of $\tilde{K}(h)$; it is a convex compact of $\mathbb{R}^{2}$. Theorem 7.6 easily yields the following estimate for the counting function :

Theorem 7.7 Denote by $N_{h}(K)$ the number of eigenvalues belonging to $h K$ :

$$
N_{h}(K) \stackrel{\text { def }}{=} \#\left\{\left(E_{1}, E_{2}\right) \in h K \cap \Sigma(h)\right\} .
$$

The following estimate holds, as $h$ tends to zero :

$$
N_{h}(K)=\frac{|\ln h|}{2 \pi} \int_{\tilde{K}_{0}}|d x \wedge d n|+O(1) .
$$

The measure $|d n|$ is the counting measure on $\mathbb{Z}$, and $|d x|$ is the standard one-dimensional Lebesgue measure.

We present in figure 12 a numerical illustration of this result.

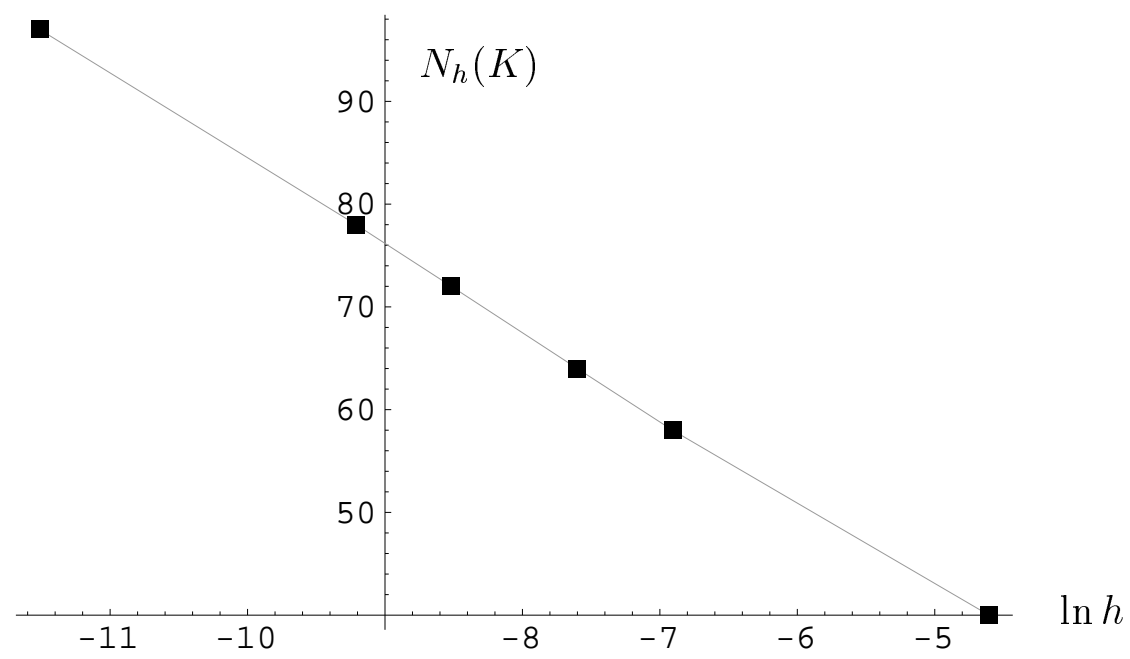

Figure 12: The counting function $N_{h}(K)$ for the Champagne bottle, where $K$ is a compact selecting only joint eigenvalues on the line $\epsilon_{2}=0$ (numerics).

The question that this theorem arouses is whether the formula for $N_{h}$ can be related to a Weyl-type heuristic involving volume in phase space. The answer is positive (proposition 7.8), up to the difference between the measures of $\tilde{K}_{0}$ with respect to $|d x \wedge d n|$ and with respect to the standard Lebesgue measure on $\mathbb{R}^{2}$, which of course strongly depends on the geometry of $\tilde{K}_{0}$.

Let $\mu$ be the push-forward on $\mathbb{R}^{2}$ of the symplectic (or Liouville) measure by the momentum $\operatorname{map} p=\left(p_{1}, p_{2}\right)$ : for any Borel $\mathcal{B}$ of $\mathbb{R}^{2}$,

$$
\mu(\mathcal{B})=\int_{p^{-1}(\mathcal{B})}|\omega \wedge \omega| / 2
$$

Let $|d c|=\left|d c_{1} \wedge d c_{2}\right|$ be the standard Lebesgue measure on $\mathbb{R}^{2}$. The Lebesgue volume of $\mathcal{B}$ will be denoted by $|\mathcal{B}|$. 
Proposition 7.8 As $h$ tends to zero,

$$
\frac{\mu(h K)}{(2 \pi h)^{2}}=\frac{|\ln h|}{2 \pi}\left|\tilde{K}_{0}\right|+O(1)
$$

Proof. Let $\Omega$ be a neighborhood of the critical point $m$ in which the system $\left(P_{1}, P_{2}\right)$ can be brought to the normal form of lemma 6.5 , so that there is a local diffeomorphism $F$ defined on $U=p(\Omega)$ such that, with the same notations as in section $6.2,\left(p_{1}, p_{2}\right)=F\left(q_{1}, q_{2}\right)$.

Replacing $K$ by $h_{0} K$ with $h_{0}$ small enough, if necessary, we can always assume that $K \subset U$. Let $\varepsilon>0$ be such that the ball

$$
B_{\varepsilon} \stackrel{\text { def }}{=}\{(r, \theta, \rho, \alpha) \in M, \quad r<\varepsilon, \rho<\varepsilon\}
$$

is included in $\Omega$. Then $\mu(h K)$ can be split into two parts :

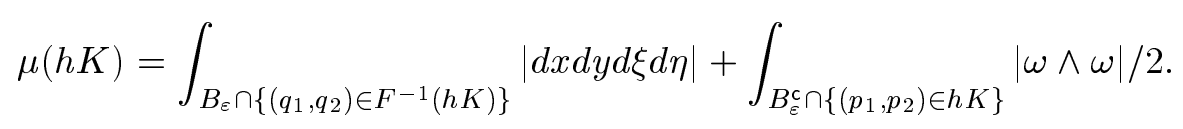

If $h$ is small enough, each fiber $p^{-1}(c), c \in h K$ intersects $B_{\varepsilon}$, and we know from section 6.4 that the fibration $p_{\left\lceil B_{\varepsilon}^{c}\right.}$ over $h K$ is trivial with fibers diffeomorphic to compact cylinders. Moreover, $p$ naturally defines on each cylinder a locally invariant measure $\boldsymbol{\rho}$ such that the symplectic measure splits according to :

$$
|\omega \wedge \omega| / 2=\boldsymbol{\rho} \otimes\left|d p_{1} \wedge d p_{2}\right| .
$$

Therefore, the second term of (27) can be written as :

$$
\int_{B_{\varepsilon}^{c} \cap\left\{\left(p_{1}, p_{2}\right) \in h K\right\}}|\omega \wedge \omega| / 2=\int_{\left(c_{1}, c_{2}\right) \in h K} \rho\left(B_{\varepsilon}^{\mathrm{c}} \cap p^{-1}(c)\right)\left|d c_{1} \wedge d c_{2}\right| .
$$

Because $\boldsymbol{\rho}\left(B_{\varepsilon}^{\mathrm{c}} \cap p^{-1}(c)\right)$ is bounded for bounded $\left(c_{1}, c_{2}\right)$, the following estimate holds, for some $C>0$ :

$$
\int_{B_{\varepsilon}^{c} \cap\left\{\left(p_{1}, p_{2}\right) \in h K\right\}}|\omega \wedge \omega| / 2<C . \int_{\left(c_{1}, c_{2}\right) \in h K}\left|d c_{1} \wedge d c_{2}\right|=C \cdot h^{2}|K| .
$$

Let us turn now to the first term in the sum (27):

$$
I_{\varepsilon}(h) \stackrel{\text { def }}{=} \int_{B_{\varepsilon} \cap\left\{\left(q_{1}, q_{2}\right) \in F^{-1}(h K)\right\}}|d x d y d \xi d \eta| .
$$

Using the new coordinates $(r, \theta, \ell, \lambda)$ with $\ell=r \rho$ and $\lambda=\alpha-\theta$, the symplectic measure is transformed into

$$
\frac{\ell}{r}|d \lambda \wedge d \ell \wedge d \theta \wedge d r|=\frac{1}{r}\left|d q_{1} \wedge d q_{2}\right| \otimes|d \theta \wedge d r|
$$

This gives:

$$
I_{\varepsilon}(h)=2 \pi \int_{\left(c_{1}, c_{2}\right) \in F^{-1}(h K)} 1_{|c| \leqslant \varepsilon^{2}}\left(\int_{\frac{|c|}{\varepsilon}}^{\varepsilon} \frac{1}{r}|d r|\right)\left|d c_{1} \wedge d c_{2}\right|
$$




$$
=2 \pi \int_{F^{-1}(h K)} 1_{|c| \leqslant \varepsilon^{2}} \ln \left(\frac{\varepsilon^{2}}{|c|}\right)\left|d c_{1} \wedge d c_{2}\right|
$$

Note that for $h$ small enough, the condition $|c| \leqslant \varepsilon^{2}$ is always satisfied for $c \in F^{-1}(h K)$.

Now, the change of variables $\hat{c}=F(c) / h$ yields

$$
I_{\varepsilon}(h)=2 \pi h^{2} \int_{\hat{c} \in K} \ln \left(\frac{\varepsilon^{2}}{\left|F^{-1}(h \hat{c})\right|}\right) J(h \hat{c})|d \hat{c}|,
$$

where $J$ is the Jacobian of $F^{-1}$. Taylor's formula for $J$ and $F^{-1}$ easily shows that the integrand can be reduced to its principal part:

$$
I_{\varepsilon}(h)=2 \pi h^{2} \int_{\hat{c} \in K} \ln \left(\frac{\varepsilon^{2}}{\left|M^{-1} \cdot h \hat{c}\right|}\right) J(0)|d \hat{c}|+O\left(h^{3}\right) .
$$

Since $J(0)=\operatorname{det} M^{-1}$, the right hand-side is equal to :

$$
2 \pi h^{2} \int_{\tilde{c} \in M^{-1}(K)} \ln \left(\frac{\varepsilon^{2}}{|h \tilde{c}|}\right)|d \tilde{c}|+O\left(h^{3}\right),
$$

with $\tilde{c}=M^{-1} \hat{c}$. Because the measure $\ln |c||d c|$ is locally integrable at the origin, this finally gives :

$$
I_{\varepsilon}(h)=-2 \pi h^{2} \ln h\left|M^{-1}(K)\right|+O\left(h^{2}\right),
$$

thus proving proposition 7.8 .

\section{References}

[1] S. Bates and A. Weinstein. Lectures on the Geometry of Quantization, volume 8 of Berkeley Mathematics Lecture Notes. AMS, 1997.

[2] S. Bochner. Theta relations with spherical harmonics. Proc. Nat. Acad. Sci. Wash., 57:804808, 1951.

[3] R. Brummelhuis, T. Paul, and A. Uribe. Spectral estimates around a critical level. Duke Mathematical Journal, 78(3):477-530, 1995.

[4] J.W.S. Cassels and A. Fröhlich. Algebraic Number Theory. Academic Press, 1967.

[5] A.-M. Charbonnel. Comportement semi-classique du spectre conjoint d'opérateurs pseudodifférentiels qui commutent. Asymptotic Analysis, 1:227-261, 1988.

[6] A.-M. Charbonnel and G. Popov. A semi-classical trace formula for several commuting operators. Preprint Univ. Nantes, 1997.

[7] M.S. Child. Quantum states in a Champagne bottle. J.Phys.A., 31:657-670, 1998.

[8] Y. Colin de Verdière. Quasi-modes sur les variétés riemanniennes. Inventiones Mathematicce, 43:15-52, 1977. 
[9] Y. Colin de Verdière. Spectre conjoint d'opérateurs pseudo-différentiels qui commutent II. Mathematische Zeitschrift, 171:51-73, 1980.

[10] Y. Colin de Verdière. Cours de DEA. Université Grenoble I, 1992.

[11] Y. Colin de Verdière and B. Parisse. Équilibre instable en régime semi-classique I : Concentration microlocale. Communications in partial differential equations, 19(9-10):1535-1563, 1994 .

[12] Y. Colin de Verdière and B. Parisse. Équilibre instable en régime semi-classique II : Conditions de Bohr-Sommerfeld. Annales de l'Institut Henri Poincaré, Physique Théorique, 61(3):347-367, 1994.

[13] Y. Colin de Verdière and B. Parisse. Conditions de Bohr-Sommerfeld singulières. Preprint Institut Fourier 432, http://www-fourier.ujf-grenoble.fr/PREP/prep_if .html, 1998.

[14] S. Colombo. Les transformations de Mellin et Hankel. CNRS, 1959.

[15] R. Cushman and J.J. Duistermaat. The quantum spherical pendulum. Bulletin of the AMS (new series), 19:475-479, 1988.

[16] R. Cushman and J.J. Duistermaat. Non-hamiltonian monodromy. preprint University of Utrecht, 1997.

[17] R. Dautray and J.-L. Lions. Transformations, Sobolev, Opérateurs, volume 3 of Analyse mathématique et calcul numérique. Masson, 1987.

[18] J.J. Duistermaat. Oscillatory integrals, Lagrange immersions and unfoldings of singularities. Comm. Pure Appl. Math., 27:207-281, 1974.

[19] J.J. Duistermaat. On global action-angle variables. Comm. Pure Appl. Math., 33:687-706, 1980.

[20] J.J. Duistermaat and G.J. Heckman. On the variation in the cohomology of the symplectic form of the reduced phase space. Inventiones mathematica, 69:259-268, 1982.

[21] J.J. Duistermaat and L. Hörmander. Fourier integral operators II. Acta mathematica, 128:183-269, 1972.

[22] L.H. Eliasson. Hamiltonian systems with Poisson commuting integrals. PhD thesis, University of Stockholm, 1984.

[23] L.H. Eliasson. Normal forms for hamiltonian systems with Poisson commuting integrals elliptic case. Comment. Math. Helvetici, 65:4-35, 1990.

[24] M. Flato, A. Lichnerowicz, and D. Sternheimer. Crochet de Moyal-Vey et quantification. Comptes Rendus Acad. Sc. Paris, 283:19-24, 1976.

[25] A.T. Fomenko. Topological classification of integrable systems, volume 6 of Advances in soviet mathematics. AMS, 1991.

[26] I.M. Gelfand, M.I. Graev, and N.Ja. Vilenkin. Les Distributions, volume 5. Dunod, 1962. 
[27] I.M. Gelfand and Z.Ia. Schapiro. Homogeneous distributions and applications. Ouspehi Mat. Naouk, 10(3):3-70, 1955. in Russian.

[28] B. Helffer and D. Robert. Comportement semi-classique du spectre des hamiltoniens quantiques elliptiques. Annales de l'Institut Fourier, 31(3):169-223, 1981.

[29] B. Helffer and J. Sjöstrand. Multiple wells in the semi-classical limit. I. Communications in Partial Differential Equations, 9:337-408, 1984.

[30] L. Hörmander. Fourier integral operators I. Acta mathematica, 127:79-183, 1971.

[31] L. Hörmander. The analysis of Linear partial differential operators, volume I-IV. Springer, 1983-90.

[32] L.M. Lerman and Ya.L. Umanskiy. Four-dimensional integrable hamiltonian systems with simple singular points (topological aspects), volume 176 of Translations of Mathematical Monographs. AMS, 1998.

[33] C. März. Spectral asymptotics for Hill's equation near the potential maximum. Asymptotic Analysis, 5:221-267, 1992.

[34] V.P. Maslov. Théorie des perturbations et méthodes asymptotiques. Dunod, Paris, 1972.

[35] Zung Nguyên Tiên. A note on focus-focus singularities. Diff. Geom. Appl., 7(2):123-130, 1997.

[36] G. Pick. Geometrisches zur Zahlentheorie. Sitzenber. Lotos (Prague), 19:311-319, 1899.

[37] D. Robert. Autour de l'approximation semi-classique, volume 68 of Progress in Mathematics. Birkhäuser, 1987.

[38] J. Sjöstrand. Semi-excited states in nondegenerate potential wells. Asymptotic Analysis, 6:29-43, 1992.

[39] S. Vũ Ngọc. Formes normales semi-classiques des systèmes complètement intégrables au voisinage d'un point critique de l'application moment. preprint Institut Fourier 377, 1997.

[40] S. Vũ Ngọc. Quantum monodromy in integrable systems. preprint Utrecht University 1055, 1998.

[41] A. Weinstein. Symplectic manifolds and their lagrangian submanifolds. Advances in Mathematics, 6:329-346, 1971.

[42] A. Weinstein. Connections of Berry and Hannay type for moving lagrangean submanifolds. Advances in Mathematics, 82:135-159, 1990.

[43] J. Williamson. On the algebraic problem concerning the normal form of linear dynamical systems. American journal of mathematics, 58(1):141-163, 1936.

[44] Maorong Zou. Monodromy in two degrees of freedom integrable systems. Journal of Geometry and Physics, 10:37-45, 1992. 